WEBER BIZARRIAS DE MELO

\title{
EFEITOS DA PRESSÃO DO SISTEMA DE ARREFECIMENTO E DA CONCENTRAÇÃO DE ETILENOGLICOL SOBRE AS CARACTERÍSTICAS DE CAVITAÇÃO DE UMA BOMBA D’ÁGUA AUTOMOTIVA
}

Exame de Qualificação apresentado à Escola Politécnica da Universidade de São Paulo para obtenção do Título de Mestre em Engenharia Mecânica

Área de concentração:

Energia e Fluidos

Orientador:

Prof. Dr. Douglas Lauria

SÃO PAULO 
WEBER BIZARRIAS DE MELO

\section{EFEITOS DA PRESSÃO DO SISTEMA DE ARREFECIMENTO E DA CONCENTRAÇÃO DE ETILENO GLICOL SOBRE AS CARACTERÍSTICAS DE CAVITAÇÃO DE UMA BOMBA D’ÁGUA AUTOMOTIVA}

Exame de Qualificação apresentado à Escola Politécnica da Universidade de São Paulo para obtenção do Título de Mestre em Engenharia Mecânica

Área de concentração:

Energia e Fluidos

Orientador:

Prof. Dr. Douglas Lauria

SÃO PAULO 


\section{FICHA CATALOGRÁFICA}

Melo, Weber Bizarrias de

Efeitos da pressão do sistema de arrefecimento e da concentração de etileno glicol sobre as características de cavitação de uma bomba d'água automotiva / W.B. de Melo. -- São Paulo, 2008.

p.

Dissertação (Mestrado) - Escola Politécnica da Universidade de São Paulo. Departamento de Engenharia Mecânica.

1. Cavitação I.Universidade de São Paulo. Escola Politécnica. Departamento de Engenharia Mecânica Il.t. 


\section{AGRADECIMENTOS}

Tenho muito a agradecer, chegando ao final de mais uma etapa dos meus estudos e da minha vida.

À minha família, pelo suporte e apoio incondicional. Ao meu pai, por acreditar tanto nas possibilidades de construção ao longo da vida, e por me fazer buscar sempre essas possibilidades. A minha mãe por participar sempre de cada passo meu, e pelo amor e orientação ao longo do meu caminho, a minha irmã, pelo amor, cumplicidade e amizade que temos.

Ao Professor Douglas Lauria, por ter aceitado partilhar esse trabalho, pela paciência e orientação.

À divisão de bombas da Dana Corporation por ter sido onde tudo começou, e em especial ao Ayres de Andrade Filho, pelo apoio e pelos recursos cedidos.

À Escola Politécnica da Universidade de São Paulo onde concluo mais uma etapa, por todas as oportunidades oferecidas e por todo o conhecimento nela disponibilizado. 
"No meio de qualquer dificuldade encontra-se a oportunidade".

Albert Einstein 


\section{RESUMO}

Este trabalho apresenta a analise do efeito da concentração da mistura etilenoglicol / água, pressão do sistema, rotação da bomba e temperatura, sobre o fenômeno cavitação em sistemas de arrefecimento, tendo dois principais objetivos:

1 - Explorar as principais características do fenômeno cavitação, através da analise crítica e citação de publicações existentes;

2 - Mapear as condições de trabalho de um sistema de arrefecimento, para então simular em bancada as interações da variação dos fatores citados acima, comparando os resultados com publicações já existentes, contribuindo então com um banco de dados que possibilite a otimização do dimensionamento de novos sistemas de arrefecimento. 


\begin{abstract}
This work presents the effect analysis of the water / ethyleneglycol mixture, system pressure, pump speed and temperature upon the cavitation phenomenon in cooling systems. Moreover, it has two main targets which are:

1 - To explore the main features of the cavitation phenomenon through a critical analysis and citation of available publications;

2 - To monitor the cooling system work conditions in order to simulate in a test rig the variation of the parameters described above, understanding its interaction, for than to provide a database that make possible the design optimization of new cooling systems.
\end{abstract}




\section{SUMÁRIO}

\section{LISTA DE FIGURAS \\ LISTA DE TABELAS \\ LISTA DE SÍMBOLOS}

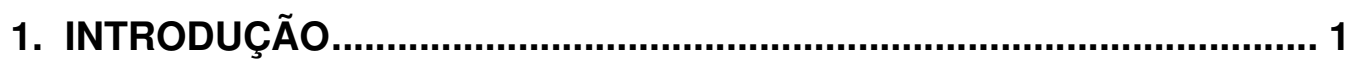

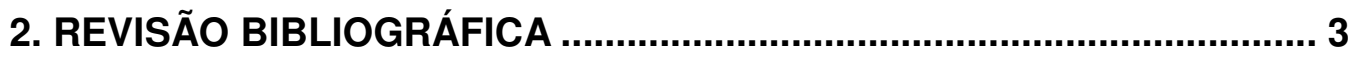

2.1 O Fenômeno Cavitação, os seus Danos e o Desempenho da Bomba 3

2.2 Efeitos da Mistura Água e Etilenoglicol, Temperatura do Fluido e

Pressão do Sistema

.14

\section{AVALIAÇÃO EXPERIMENTAL DA INFLUÊNCIA DA CONCENTRAÇÃO DE ETILENOGLICOL NA TEMPERATURA DE CAVITAÇÃO .....................22}

3.1 Medição dos Parâmetros de Trabalho do Sistema de Arrefecimento .22

3.2 Bancada de Teste .28

3.3 Simulação em bancada da temperatura de cavitação do sistema de arrefecimento 34

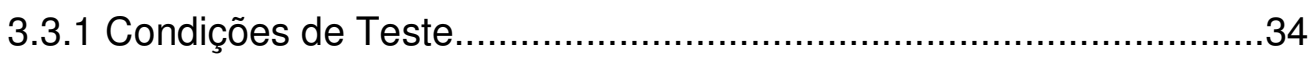

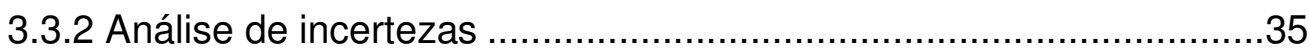

3.4.1 Incertezas Associadas ao Mapeamento dos Parâmetros de

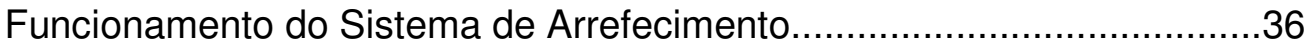

3.4.2 Incertezas Associadas a Simulação em Bancada.............................37 Incerteza Composta Associada à Medição do Diferencial de Pressão: ....37

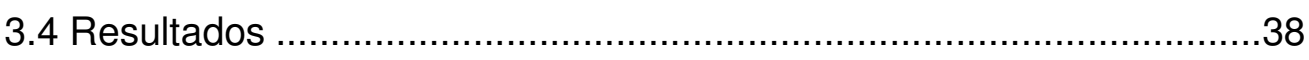

4. CONCLUSÕES

ANEXO A

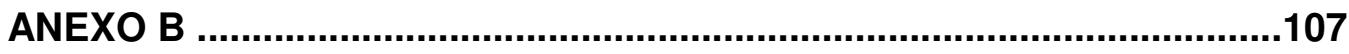

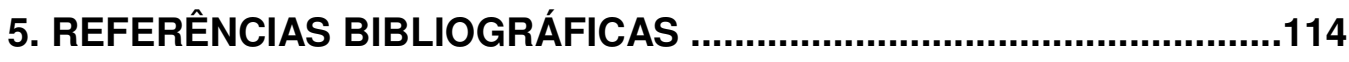




\section{LISTA DE FIGURAS}

Figura 1 - Ressurgimento das Bolhas - Adaptação de ELLIS (1966) ........... 4

Figura 2- Colapso de uma bolha de cavitação, próxima a uma superfície sólida. Adaptação de Brennen (2007)............................................... 5

Figura 3- Ilustração de um Micro-Jato. Adaptação de Brennen (2007)......... 5

Figura 4 - Desenvolvimento de um micro-jato. Lauterborn (2001) ................ 6

Figura 5 - Bomba de um sistema de arrefecimento, que sofreu danos por cavitação. Imagens gentilmente cedidas por Lauria, D........................... 7

Figura 6 - Capa do rotor danificada por cavitação. Imagem gentilmente

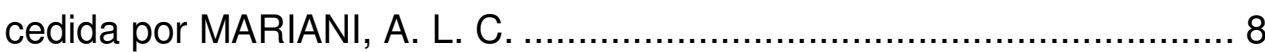

Figura 7 - Condição de Montagem da Bomba Danificada............................ 8

Figura 8 - Monitoramento de pressões ao longo do colapso de uma bolha de

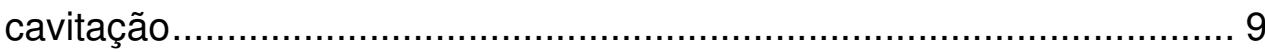

Figura 9 - Taxa Teórica de Erosão x NPSH ...........................................12

Figura 10 - Taxa de Erosão x NPSH, Chan (1988) …………………......12

Figura 11 - Curva de Perda de Massa ( $\sigma=0.015$ ) - Hattori (2006) .............15

Figura 12 - Curvas de MDER - Hattori (2006).........................................16

Figura 13 - Relação entre Temperatura e MDER máximo - Hattori (2006). 17

Figura 14 - Intensidade de Cavitação em Função da Temperatura - Hosny (2006).

Figura 15- Intensidade de Cavitação em Função da Concentração de

Etilenoglicol - Hosny (2006).

Figura 16 - Efeito da Concentração de Glicol na Erosão por Cavitação -

Hosny (2006).

Figura 17 - Instrumentação do Motor ..................................................22

Figura 18 - Termopar Montado no By-Pass da Válvula Termostática...........23

Figura 19 - Termopar Montado na Saída do Radiador................................23

Figura 20 - Transdutor de Pressão ..........................................................24

Figura 21 - Módulos para Conversão dos Sinais ......................................24

Figura 22 - Ponto de Tomada das Pressões do Sistema............................. 25 
Figura 23 - Monitoramento de Pressões e Temperaturas do Sistema de

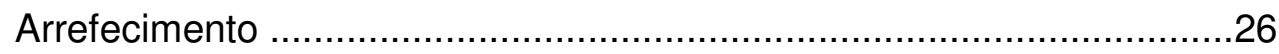

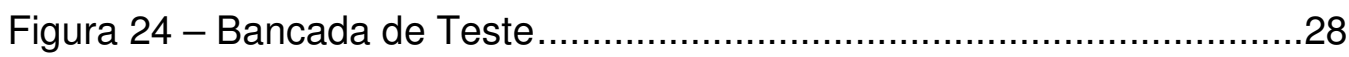

Figura 25 - Reservatório, Válvula Proporcional, Resistências, e Linhas de Ar

Comprimido e Água de Refrigeração.................................................29

Figura 26 - Medidor de Vazão Eletromagnético ........................................30

Figura 27 - Termopares e Transdutores de Pressão .................................30

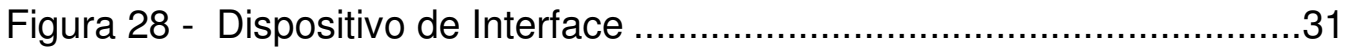

Figura 29 - Motor e Inversor de Freqüência.............................................32

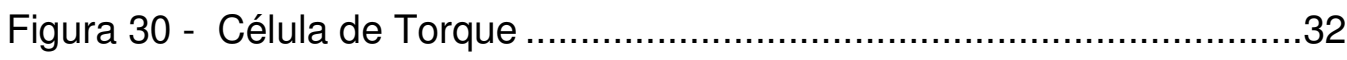

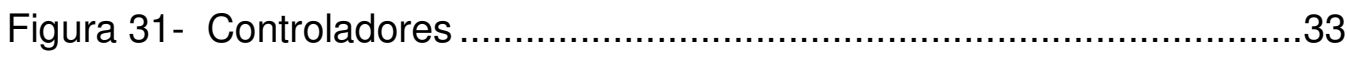

Figura 32 - Mistura de 50\% Etilenoglicol e 50\% água, com pressão de

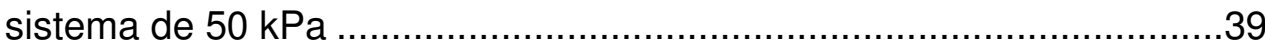

Figura 33 - Temperatura de cavitação para a mistura 50\% etilenoglicol e $50 \%$ água, a $50 \mathrm{kPa}$ de pressão de sistema, ponderando as incertezas

Figura 34 - Mistura de 50\% Etilenoglicol e 50\% água, com pressão de sistema de $15 \mathrm{kPa}$

Figura 35 - Temperatura de cavitação para a mistura 50\% etilenoglicol e $50 \%$ água, a $15 \mathrm{kPa}$ de pressão de sistema, ponderando as incertezas

Figura 36 - Mistura de 30\% Etilenoglicol e 70\% água, com pressão de sistema de $50 \mathrm{kPa}$

Figura 37 - Temperatura de cavitação para a mistura 30\% etilenoglicol e $70 \%$ água, a $50 \mathrm{kPa}$ de pressão de sistema, ponderando as incertezas.

Figura 38- Mistura de 30\% Etilenoglicol e 70\% água, com pressão de sistema de $15 \mathrm{kPa}$ .44

Figura 39 - Temperatura de cavitação para a mistura 30\% etilenoglicol e $70 \%$ água, a $15 \mathrm{kPa}$ de pressão de sistema, ponderando as incertezas. 
Figura 40-100\% água, com pressão de sistema de $50 \mathrm{kPa}$

Figura 41 - Temperatura de cavitação para 100\% água, a $50 \mathrm{kPa}$ de pressão de sistema, ponderando as incertezas....................................47

Figura $42-100 \%$ água, com pressão de sistema de $15 \mathrm{kPa}$.......................47

Figura 43 - Temperatura de cavitação para 100\% água, a $50 \mathrm{kPa}$ de pressão de sistema, ponderando as incertezas.....................................48

Figura 44 - Bomba a 5000 rpm e sistema com $50 \mathrm{kPa}$.............................49

Figura 46 - Bomba a 4000 rpm e sistema com $50 \mathrm{kPa}$..............................50

Figura 47 - Bomba a 4000 rpm e sistema com $15 \mathrm{kPa}$...............................51

Figura 48 - Bomba a 3000 rpm e sistema com $50 \mathrm{kPa}$...............................51

Figura 49 - Bomba a 3000 rpm e sistema com $15 \mathrm{kPa}$............................52 


\section{LISTA DE TABELAS}

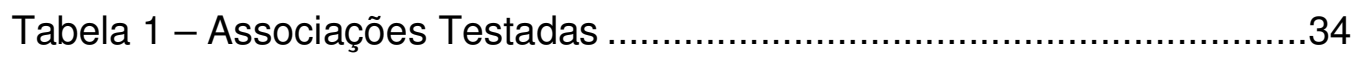

Tabela 2 - Fator de Abrangência...........................................................36

Tabela A1 - Incerteza combinada para 1000 rpm, água com 15 kPa de pressão de sistema.................................................................56

Tabela A2 - Coeficientes de sensibilidade para a pressão de entrada a 1000 rpm, água com $15 \mathrm{kPa}$ de pressão de sistema................................57

Tabela A3 - Coeficientes de sensibilidade para a pressão de saída a 1000 rpm, água com $15 \mathrm{kPa}$ de pressão de sistema................................57

Tabela A4 - Incerteza combinada para 2000 rpm, água com 15 kPa de pressão de sistema................................................................58

Tabela A5 - Coeficientes de sensibilidade para a pressão de entrada a 2000 rpm, água com $15 \mathrm{kPa}$ de pressão de sistema.

Tabela A6 - Coeficientes de sensibilidade para a pressão de saída a 2000rpm,água com $15 \mathrm{kPa}$ de pressão de sistema.

Tabela A7 - Incerteza combinada para 3000 rpm, água com 15 kPa de pressão de sistema.

Tabela A8 - Coeficientes de sensibilidade para a pressão de entrada a 3000 rpm, água com $15 \mathrm{kPa}$ de pressão de sistema.

Tabela A9 - Coeficientes de sensibilidade para a pressão de saída a 3000 rpm, água com $15 \mathrm{kPa}$ de pressão de sistema.

Tabela A10 - Incerteza combinada para 4000 rpm, água com 15 kPa de pressão de sistema.

Tabela A11 - Coeficientes de sensibilidade para a pressão de entrada a 4000 rpm, água com 15 kPa de pressão de sistema.

Tabela A12 - Coeficientes de sensibilidade para a pressão de saída a 4000 rpm, água com $15 \mathrm{kPa}$ de pressão de sistema.

Tabela A13 - Incerteza combinada para 5000 rpm, água com 15 kPa de pressão de sistema.

Tabela A14 - Coeficientes de sensibilidade para a pressão de entrada a 5000 rpm, água com 15 kPa de pressão de sistema. 64 
Tabela A15 - Coeficientes de sensibilidade para a pressão de saída a 5000 rpm, água com $15 \mathrm{kPa}$ de pressão de sistema.

Tabela A16 - Incerteza combinada para 1000 rpm, água com 50 kPa de pressão de sistema.

Tabela A17 - Coeficientes de sensibilidade para a pressão de entrada a 1000 rpm, água com $50 \mathrm{kPa}$ de pressão de sistema.

Tabela A18 - Coeficientes de sensibilidade para a pressão de saída a 1000 rpm, água com $50 \mathrm{kPa}$ de pressão de sistema. .66

Tabela 21 - Incerteza combinada para 2000 rpm, água com 50 kPa de pressão de sistema.

Tabela A20 - Coeficientes de sensibilidade para a pressão de entrada a 2000 rpm, água com $50 \mathrm{kPa}$ de pressão de sistema.

Tabela A21 - Coeficientes de sensibilidade para a pressão de saída a 2000 rpm, água com $50 \mathrm{kPa}$ de pressão de sistema.

Tabela A22 - Incerteza combinada para 3000 rpm, água com 50 kPa de pressão de sistema.

Tabela A23 - Coeficientes de sensibilidade para a pressão de entrada a 3000 rpm, água com $50 \mathrm{kPa}$ de pressão de sistema.

Tabela A24 - Coeficientes de sensibilidade para a pressão de saída a 3000 rpm, água com $50 \mathrm{kPa}$ de pressão de sistema.

Tabela A25 - Incerteza combinada para 4000 rpm, água com 50 kPa de pressão de sistema.

Tabela A26 - Coeficientes de sensibilidade para a pressão de entrada a $4000 \mathrm{rpm}$, água com $50 \mathrm{kPa}$ de pressão de sistema.

Tabela A7 - Coeficientes de sensibilidade para a pressão de saída a 4000 rpm, água com $50 \mathrm{kPa}$ de pressão de sistema.

Tabela A28 - Incerteza combinada para 5000 rpm, água com $50 \mathrm{kPa}$ de pressão de sistema.

Tabela A29 - Coeficientes de sensibilidade para a pressão de entrada a 5000 rpm, água com $50 \mathrm{kPa}$ de pressão de sistema. 
Tabela A30 - Coeficientes de sensibilidade para a pressão de saída a 5000 rpm, água com $50 \mathrm{kPa}$ de pressão de sistema.

Tabela A31 - Incerteza combinada para 1000 rpm, mistura 30/70 com 15 kPa de pressão de sistema.

Tabela A32 - Coeficientes de sensibilidade para a pressão de entrada a $1000 \mathrm{rpm}$, mistura $30 / 70 \mathrm{com} 15 \mathrm{kPa}$ de pressão de sistema.

Tabela A33 - Coeficientes de sensibilidade para a pressão de saída a 1000 rpm, mistura 30/70 com $15 \mathrm{kPa}$ de pressão de sistema. .74

Tabela A34 - Incerteza combinada para 2000 rpm, mistura 30/70 com 15 kPa de pressão de sistema. .75

Tabela A35 - Coeficientes de sensibilidade para a pressão de entrada a $2000 \mathrm{rpm}$, mistura 30/70 com $15 \mathrm{kPa}$ de pressão de sistema.

Tabela A36 - Coeficientes de sensibilidade para a pressão de saída a 2000 rpm, mistura 30/70 com $15 \mathrm{kPa}$ de pressão de sistema. .76

Tabela A37- Incerteza combinada para 3000 rpm, mistura $30 / 70$ com $15 \mathrm{kPa}$ de pressão de sistema...................................................................

Tabela A38- Coeficientes de sensibilidade para a pressão de entrada a 3000 rpm, mistura 30/70 com $15 \mathrm{kPa}$ de pressão de sistema.

Tabela A39 - Coeficientes de sensibilidade para a pressão de saída a 3000 rpm, mistura 30/70 com $15 \mathrm{kPa}$ de pressão de sistema. .78

Tabela A40 - Incerteza combinada para 4000 rpm, mistura 30/70 com 15 kPa de pressão de sistema.

Tabela A41 - Coeficientes de sensibilidade para a pressão de entrada a $4000 \mathrm{rpm}$, mistura $30 / 70 \mathrm{com} 15 \mathrm{kPa}$ de pressão de sistema.

Tabela A42 - Coeficientes de sensibilidade para a pressão de saída a 4000 rpm, mistura 30/70 com $15 \mathrm{kPa}$ de pressão de sistema.

Tabela A43 - Incerteza combinada para 5000 rpm, mistura 30/70 com 15 kPa de pressão de sistema.

Tabela A44 - Coeficientes de sensibilidade para a pressão de entrada a $5000 \mathrm{rpm}$, mistura $30 / 70 \mathrm{com} 15 \mathrm{kPa}$ de pressão de sistema. 
Tabela A45 - Coeficientes de sensibilidade para a pressão de saída a 5000 rpm, mistura 30/70 com $15 \mathrm{kPa}$ de pressão de sistema.

Tabela A46 - Incerteza combinada para 1000 rpm, mistura 30/70 com 50 kPa de pressão de sistema.

Tabela A46 - Coeficientes de sensibilidade para a pressão de entrada a $1000 \mathrm{rpm}$, mistura $30 / 70 \mathrm{com} 50 \mathrm{kPa}$ de pressão de sistema.

Tabela A47 - Coeficientes de sensibilidade para a pressão de saída a 1000 rpm, mistura 30/70 com $50 \mathrm{kPa}$ de pressão de sistema.

Tabela A48 - Incerteza combinada para 2000 rpm, mistura 30/70 com 50 $\mathrm{kPa}$ de pressão de sistema.

Tabela A49 - Coeficientes de sensibilidade para a pressão de entrada a 2000 rpm, mistura 30/70 com $50 \mathrm{kPa}$ de pressão de sistema.

Tabela A50 - Coeficientes de sensibilidade para a pressão de saída a 2000 rpm, mistura 30/70 com $50 \mathrm{kPa}$ de pressão de sistema. .85

Tabela A51 - Incerteza combinada para 3000 rpm, mistura 30/70 com 50 $\mathrm{kPa}$ de pressão de sistema. .86

Tabela A52 - Coeficientes de sensibilidade para a pressão de entrada a $3000 \mathrm{rpm}$, mistura 30/70 com $50 \mathrm{kPa}$ de pressão de sistema.

Tabela A53 - Coeficientes de sensibilidade para a pressão de saída a 3000 rpm, mistura 30/70 com $50 \mathrm{kPa}$ de pressão de sistema.

Tabela A54 - Incerteza combinada para 4000 rpm, mistura 30/70 com 50 kPa de pressão de sistema.

Tabela A55 - Coeficientes de sensibilidade para a pressão de entrada a 4000 rpm, mistura 30/70 com $50 \mathrm{kPa}$ de pressão de sistema.

Tabela A56 - Coeficientes de sensibilidade para a pressão de saída a 4000 rpm, mistura 30/70 com $50 \mathrm{kPa}$ de pressão de sistema.

Tabela A57 - Incerteza combinada para 5000 rpm, mistura 30/70 com 50 $\mathrm{kPa}$ de pressão de sistema.

Tabela A8 - Coeficientes de sensibilidade para a pressão de entrada a 5000 rpm, mistura 30/70 com $50 \mathrm{kPa}$ de pressão de sistema. 90 
Tabela A59 - Coeficientes de sensibilidade para a pressão de saída a 5000 rpm, mistura 30/70 com $50 \mathrm{kPa}$ de pressão de sistema.

Tabela A60 - Incerteza combinada para 1000 rpm, mistura 50/50 com 15 kPa de pressão de sistema.

Tabela A61 - Coeficientes de sensibilidade para a pressão de entrada a $1000 \mathrm{rpm}$, mistura 50/50 com $15 \mathrm{kPa}$ de pressão de sistema.

Tabela A62 - Coeficientes de sensibilidade para a pressão de saída a 1000 rpm, mistura 50/50 com $15 \mathrm{kPa}$ de pressão de sistema. ......................92

Tabela A63 - Incerteza combinada para 2000 rpm, mistura 50/50 com 15 $\mathrm{kPa}$ de pressão de sistema. .93

Tabela A64 - Coeficientes de sensibilidade para a pressão de entrada a 2000 rpm, mistura 50/50 com $15 \mathrm{kPa}$ de pressão de sistema.

Tabela A65 - Coeficientes de sensibilidade para a pressão de saída a 2000 rpm, mistura 50/50 com $15 \mathrm{kPa}$ de pressão de sistema. .94

Tabela A66 - Incerteza combinada para 3000 rpm, mistura 50/50 com 15 $\mathrm{kPa}$ de pressão de sistema. .95

Tabela A67 - Coeficientes de sensibilidade para a pressão de entrada a $3000 \mathrm{rpm}$, mistura 50/50 com $15 \mathrm{kPa}$ de pressão de sistema.

Tabela A68 - Coeficientes de sensibilidade para a pressão de saída a 3000 rpm, mistura 50/50 com $15 \mathrm{kPa}$ de pressão de sistema. .96

Tabela A69 - Incerteza combinada para 4000 rpm, mistura 50/50 com 15 $\mathrm{kPa}$ de pressão de sistema. .96

Tabela A70 - Coeficientes de sensibilidade para a pressão de entrada a $4000 \mathrm{rpm}$, mistura 50/50 com $15 \mathrm{kPa}$ de pressão de sistema.

Tabela A71 - Coeficientes de sensibilidade para a pressão de saída a 4000 rpm, mistura 50/50 com $15 \mathrm{kPa}$ de pressão de sistema.

Tabela A72 - Incerteza combinada para 5000 rpm, mistura 50/50 com 15 $\mathrm{kPa}$ de pressão de sistema. .98

Tabela A73 - Coeficientes de sensibilidade para a pressão de entrada a $5000 \mathrm{rpm}$, mistura 50/50 com $15 \mathrm{kPa}$ de pressão de sistema. 
Tabela A74 - Coeficientes de sensibilidade para a pressão de saída a 5000 rpm, mistura 50/50 com $15 \mathrm{kPa}$ de pressão de sistema.

Tabela A75 - Incerteza combinada para 1000 rpm, mistura 50/50 com 50 kPa de pressão de sistema.

Tabela A76 - Coeficientes de sensibilidade para a pressão de entrada a $1000 \mathrm{rpm}$, mistura 50/50 com $50 \mathrm{kPa}$ de pressão de sistema.

Tabela A77 - Coeficientes de sensibilidade para a pressão de saída a 1000 rpm, mistura 50/50 com $50 \mathrm{kPa}$ de pressão de sistema.

Tabela A78 - Incerteza combinada para 2000 rpm, mistura 50/50 com 50 $\mathrm{kPa}$ de pressão de sistema

Tabela A79 - Coeficientes de sensibilidade para a pressão de entrada a 2000 rpm, mistura 50/50 com $50 \mathrm{kPa}$ de pressão de sistema.

Tabela A80 - Coeficientes de sensibilidade para a pressão de saída a 2000 rpm, mistura 50/50 com $50 \mathrm{kPa}$ de pressão de sistema. 102

Tabela A81 - Incerteza combinada para 3000 rpm, mistura 50/50 com 50 $\mathrm{kPa}$ de pressão de sistema. 102

Tabela A82 - Coeficientes de sensibilidade para a pressão de entrada a $3000 \mathrm{rpm}$, mistura 50/50 com $50 \mathrm{kPa}$ de pressão de sistema. 103

Tabela A83 - Coeficientes de sensibilidade para a pressão de entrada a $3000 \mathrm{rpm}$, mistura 50/50 com $50 \mathrm{kPa}$ de pressão de sistema. 103

Tabela A84 - Incerteza combinada para 4000 rpm, mistura 50/50 com 50 kPa de pressão de sistema. .104

Tabela A85 - Coeficientes de sensibilidade para a pressão de entrada a 4000 rpm, mistura 50/50 com $50 \mathrm{kPa}$ de pressão de sistema. .104

Tabela A86 - Coeficientes de sensibilidade para a pressão de saída a 4000 rpm, mistura 50/50 com $50 \mathrm{kPa}$ de pressão de sistema. 105

Tabela A87 - Incerteza combinada para 5000 rpm, mistura 50/50 com 50 kPa de pressão de sistema. 105

Tabela A88 - Coeficientes de sensibilidade para a pressão de entrada a 5000 rpm, mistura 50/50 com $50 \mathrm{kPa}$ de pressão de sistema. 106 
Tabela A89 - Coeficientes de sensibilidade para a pressão de entrada a 5000 rpm, mistura 50/50 com 50 kPa de pressão de sistema. ............106 


\section{LISTA DE SÍMBOLOS}

\begin{tabular}{|c|c|}
\hline$R m$ & resiliência máxima \\
\hline$\sigma r$ & tensão de ruptura \\
\hline$E$ & modulo de elasticidade \\
\hline$\omega r$ & trabalho feito para causar a fratura \\
\hline$\sigma r$ & tensão de ruptura \\
\hline$L$ & deformação \\
\hline$p$ & pressão estática \\
\hline$C p$ & coeficiente de pressão \\
\hline$\rho$ & massa especifica \\
\hline$p_{1}$ & pressão na entrada \\
\hline$U$ & velocidade na ponta da pá na aresta de sucção \\
\hline$\Omega$ & velocidade angular \\
\hline$R_{T 1}$ & raio da aresta de sucção \\
\hline$\sigma$ & número de cavitação \\
\hline NPSP & net positive suction pressure \\
\hline NPSE & net positive suction energy \\
\hline$p_{v}$ & pressão de vapor \\
\hline$p_{1}^{T}$ & pressão total na entrada \\
\hline$\sigma i$ & ponto de inicio de cavitação \\
\hline MDER & profundidade média da taxa de erosão \\
\hline$\Delta P$ & diferença de pressão \\
\hline
\end{tabular}




$\begin{array}{ll}\text { DP5\% } & \text { representa queda de } 5 \% \text { no diferencial de pressão } \\ \text { DP10\% } & \text { representa queda de } 10 \% \text { no diferencial de } \\ \text { pressão } & \text { exatidão do instrumento } \\ k & \text { fator de abrangência } \\ u & \text { incerteza } \\ u_{T} & \text { incerteza associada ao termopar } \\ u_{p} & \text { incerteza associada ao transdutor de pressão } \\ u_{c} & \text { incerteza combinada } \\ D p & \text { diferencia de pressão } \\ S & \text { coeficiente de sensibilidade } \\ S p_{1} & \text { coeficiente de sensibilidade para a pressão de } \\ \text { entrada } & \\ S p_{2} & \text { coeficiente de sensibilidade para a pressão de } \\ \text { saída } & \end{array}$




\section{INTRODUÇÃO}

Os avanços no projeto e manufatura dos motores de combustão interna têm aumentado gradualmente sua eficiência térmica e a temperatura de combustão nos cilindros. Tal fato exige uma maior atenção para o sistema de arrefecimento do motor.

Até a década de 70 os sistemas de arrefecimento eram abertos, com a água neles em circulação sendo reposta regularmente. Com a evolução dos motores passou-se a usar sistemas de arrefecimento fechados, com reservatórios intermediários de expansão e válvulas termostáticas visando permitir a operação dos motores nas temperaturas mais altas possíveis. Com o novo sistema, a retirada de calor do motor somente acontece se a temperatura ultrapassa um determinado valor limite, quando abre-se a válvula termostática e o fluido é circulado pelo sistema. Com isso, a água deixou de ser viável como fluido refrigerante para estes sistemas. O novo fluido aplicado nos sistemas de arrefecimento de motores de combustão interna é constituído por uma mistura de água e etilenoglicol, onde o último tem a função de aumentar a temperatura de ebulição e baixar a temperatura de congelamento da mistura. O sistema de arrefecimento é pressurizado pela expansão térmica do fluido de arrefecimento e o recipiente de expansão é equipado com válvula de alivio que limita a pressão interna do sistema em torno de $140 \mathrm{kPa}$ (pressão efetiva).

Os maiores problemas para um sistema de arrefecimento estão relacionados com a vaporização do líquido no seu interior e a potencial ocorrência de cavitação. Esta será conseqüente às baixas pressões em regiões definidas do circuito de arrefecimento.

Esta dissertação objetiva estudar o fenômeno de cavitação em bombas aplicadas em sistemas de arrefecimento. Objetiva ainda analisar critica e experimentalmente os parâmetros de funcionamento de sistemas de 
arrefecimento, sob o aspecto da potencialidade de ocorrência de cavitação ao se associar diferentes pressões de sistema e temperaturas, utilizando-se diferentes misturas de água e etilenoglicol.

Tais fatos serão verificados através da revisão bibliográfica de publicações referentes ao estudo do fenômeno cavitação e a análise experimental das diferentes pressões do sistema encontradas nos variados regimes de utilização de um veiculo, determinando-se então as temperaturas de cavitação para diferentes misturas de água e etilenoglicol.

A dissertação está dividida em quatro capítulos para apresentar de forma seqüencial o desenvolvimento do estudo.

O Capitulo 1 é a introdução do trabalho e apresenta o contexto na qual se insere o estudo.

No Capitulo 2 é apresentada a revisão bibliográfica, que em sua primeira parte aborda a fundamentação e as principais características do fenômeno cavitação e na segunda parte explora os efeitos da mistura etilenoglicolágua, da temperatura e da pressão do sistema de arrefecimento na ocorrência de cavitação.

O Capitulo 3 apresenta o mapeamento das condições de operação de um sistema de arrefecimento, a descrição da bancada de teste utilizada e os resultados das simulações experimentais propostas nesse estudo.

No capítulo seguinte, o Capitulo 4, é apresentada a conclusão do trabalho. 


\section{REVISÃO BIBLIOGRÁFICA}

Diversos estudos sobre cavitação e sua interação no funcionamento de bombas centrifugas estão disponíveis na literatura. Estes trabalhos levam em consideração aspectos como as circunstâncias de sua formação, os possíveis danos resultantes e seu efeito sobre o comportamento da bomba. Tais publicações apresentam a caracterização do fenômeno e avaliações sobre seu efeito no sistema de arrefecimento de motores de combustão interna ao se adicionar etilenoglicol à água.

Alguns destes trabalhos serão apresentados a seguir, separados entre a caracterização do fenômeno de cavitação e a abordagem específica dos efeitos do etilenoglicol, da temperatura do fluido e da pressão do sistema.

\subsection{O Fenômeno Cavitação, os seus Danos e o Desempenho da Bomba}

Cavitação é o processo de vaporização de líquido em regiões de baixa pressão. Apesar de ser um fenômeno dinâmico, associado ao escoamento no circuito de arrefecimento, pode-se admitir que a cavitação ocorra quando a pressão no liquido de arrefecimento atinge a pressão de vapor para a temperatura local do sistema.

Entre 1930 e 1940, Robert Knapp, professor do Instituto de Tecnologia da Califórnia, construiu a primeira câmera de alta velocidade com condições para registrar o processo de cavitação e implosões das bolhas. Em um túnel de água, ele posicionou a câmera em frente a uma região de baixa pressão. As bolhas surgiam gradualmente ao entrar nessa região, e colapsavam violentamente ao sair dela. 
A ação nefasta da cavitação ocorre quando a bolha, ao alcançar campos de maior pressão, colapsa próximo a uma superfície sólida levando à sua erosão.

Quando uma bolha colapsa, ela ressurge algumas vezes, como mostrado na figura 1. Uma bolha é constituída, em sua maior parte, por vapor de líquido que condensa quando ocorre o colapso, mas também existe na bolha uma pequena parcela de ar que pode ser comprimido, mas não pode ser eliminado. Ele eventualmente é comprimido até certo limite e então retorna a um volume prévio, fazendo com que a bolha ressurja e se divida formando uma nuvem de pequenas bolhas.

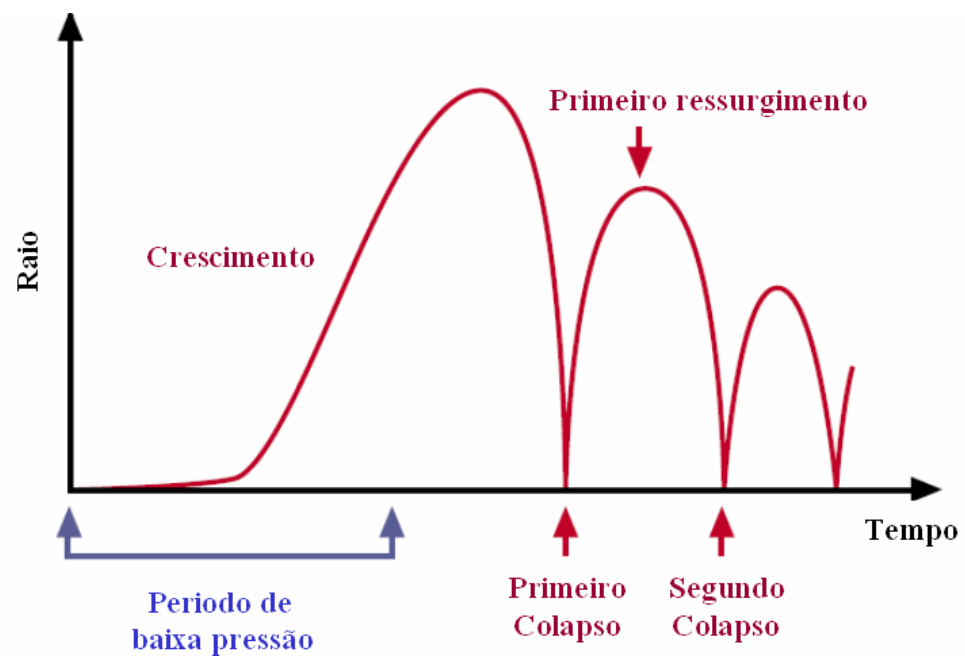

Figura 1 - Ressurgimento das Bolhas - Adaptação de ELLIS (1966)

Quando o colapso ocorre próximo a uma superfície sólida, BENJAMIN e ELLIS (1966) observaram o desenvolvimento e a formação de um micro-jato que invade a bolha a partir do ponto mais distante da superfície sólida, conforme a figura 2. Esse micro-jato alcança altas velocidades, de forma que o impacto do outro lado da bolha gera ondas de choque e o carregamento localizado da superfície próxima. Brennen (2007) entende que o micro-jato se forma porque o lado da bolha próximo à superfície sólida colapsa de forma mais lenta que o restante da bolha, pois fluido tem que se mover ao 
longo da superfície para ocupar o volume então tomado pela bolha, enquanto que de qualquer outra direção o fluido se move diretamente.

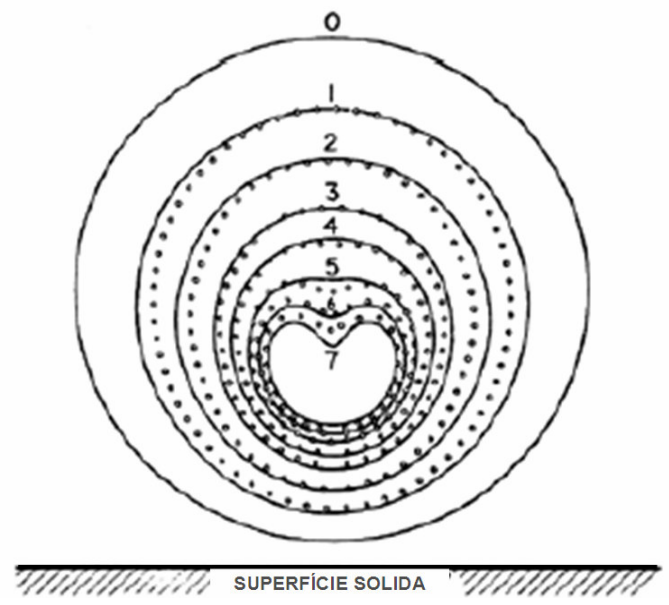

Figura 2- Colapso de uma bolha de cavitação, próxima a uma superfície sólida. Adaptação de Brennen (2007)

A Figura 3 ilustra a forma assimétrica do colapso de um micro-jato. O microjato é a coluna fina e escura indicada por uma seta no centro da bolha. A grande protuberância é o jato atravessando o fundo da bolha.

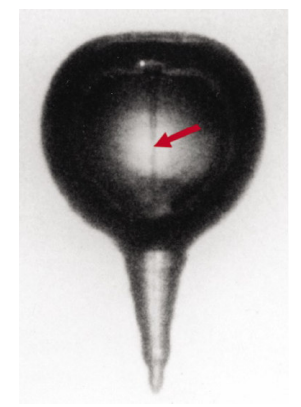

Figura 3- Ilustração de um Micro-Jato. Adaptação de Brennen (2007).

LAUTERBORN (2001) investiga a estrutura desses micro-jatos, através da observação do colapso de bolhas de cavitação com uma câmera de alta velocidade e avalia sua formação e consistência. Em seu artigo, Lauterborn 
conclui que o micro-jato é formado por minúsculas bolhas de cavitação que emergem das ondas de choque do colapso.

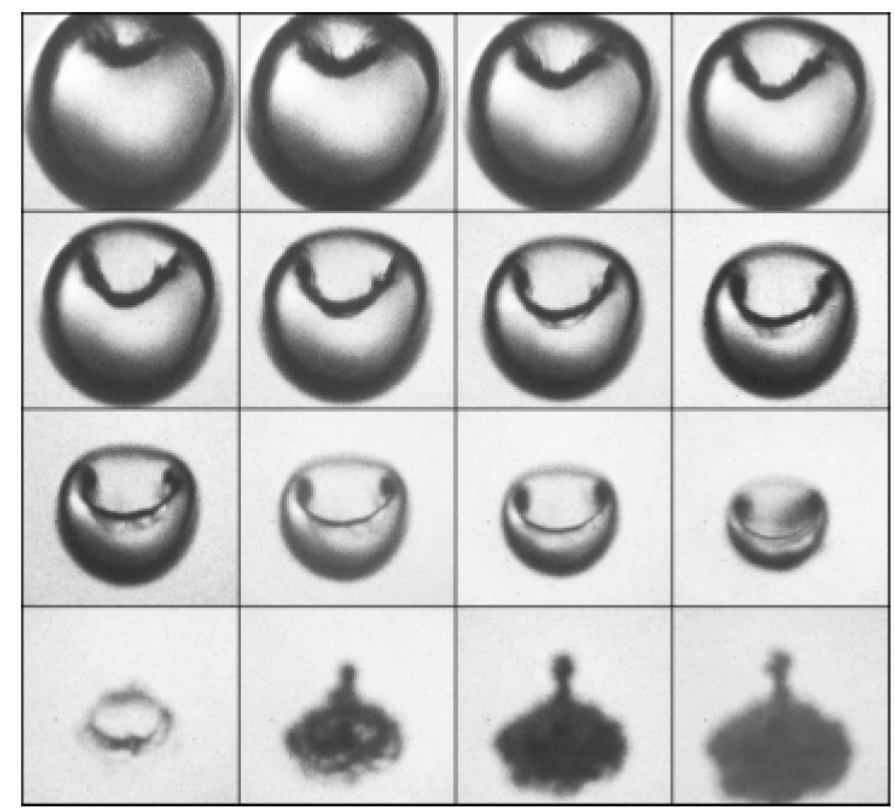

Figura 4 - Desenvolvimento de um micro-jato. Lauterborn (2001)

O desenvolvimento de um micro-jato durante o colapso de uma bolha de cavitação é visto na figura 4 . Na primeira seqüência de fotos a forma da bolha se aproxima de uma esfera. Ela tem uma superfície suave, com uma depressão no lado oposto à superfície sólida. Nessa seqüência de fotos, a bolha tem um raio de $0,6 \mathrm{~mm}$ e seu volume foi contraído a $6 \%$ da condição inicial. Nas seqüências seguintes, com a progressão do colapso, o volume da bolha diminui ainda mais. A depressão aumenta e forma o micro-jato que penetra na bolha em direção à superfície sólida. Instantes antes da primeira imagem da quarta linha, o jato rompe a parede inferior da bolha. A bolha então tem o formato de um fino anel. Na segunda imagem da quarta linha, a bolha está ressurgindo. O Micro-jato se desenvolveu e é visível no topo da bolha. Lauterborn não consegue discernir se o jato e a bolha são conectados ou não. 
O resultado das ondas de choque e dos microjatos é um processo violento que gera tensões superficiais localizadas. A repetição desse processo pode causar falhas por fadiga em pontos da superfície sólida e o desprendimento de pedaços do material. Diferentemente da erosão causada por partículas sólidas presentes no escoamento, onde a superfície parece estar levemente desgastada e com riscos, os danos por cavitação têm a aparência cristalina e irregular de falhas por fadiga.
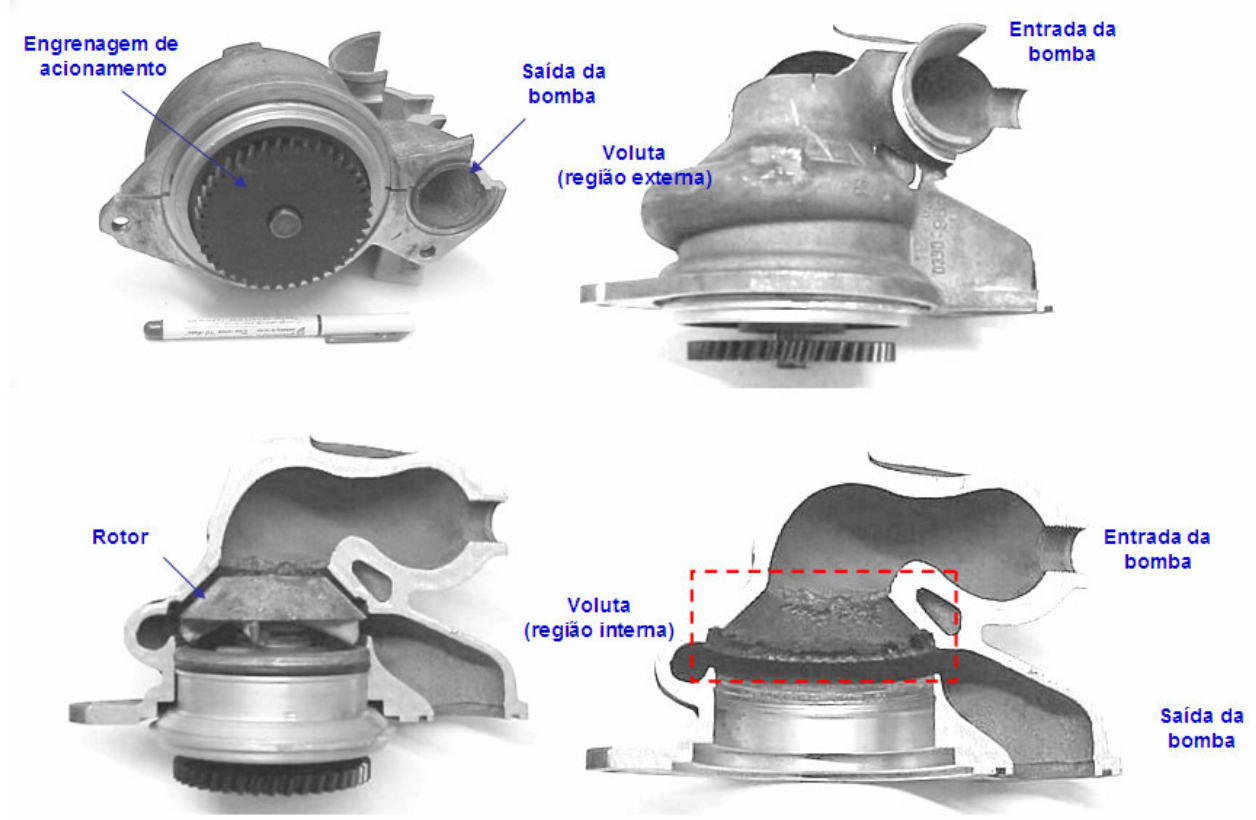

Figura 5 - Bomba de um sistema de arrefecimento, que sofreu danos por cavitação. Imagens gentilmente cedidas por Lauria, D.

A Figura 5 ilustra essa condição mostrando danos por cavitação localizada na carcaça de uma bomba d'água de um motor de combustão interna fabricada em alumínio. A engrenagem aciona o eixo do rotor, e a saliência ao lado direito da engrenagem é à saída da bomba. $O$ canto superior direito apresenta a entrada da bomba e a área externa de sua voluta, compreendida entre a engrenagem de acionamento e a entrada da bomba. Uma vista da bomba em corte é apresentada no canto inferior direito, onde é 
possível identificar o rotor, a secção transversal da voluta e das galerias de admissão e saída. No canto inferior direito, a região identificada em vermelho mostra o local onde os danos por cavitação ocorreram.

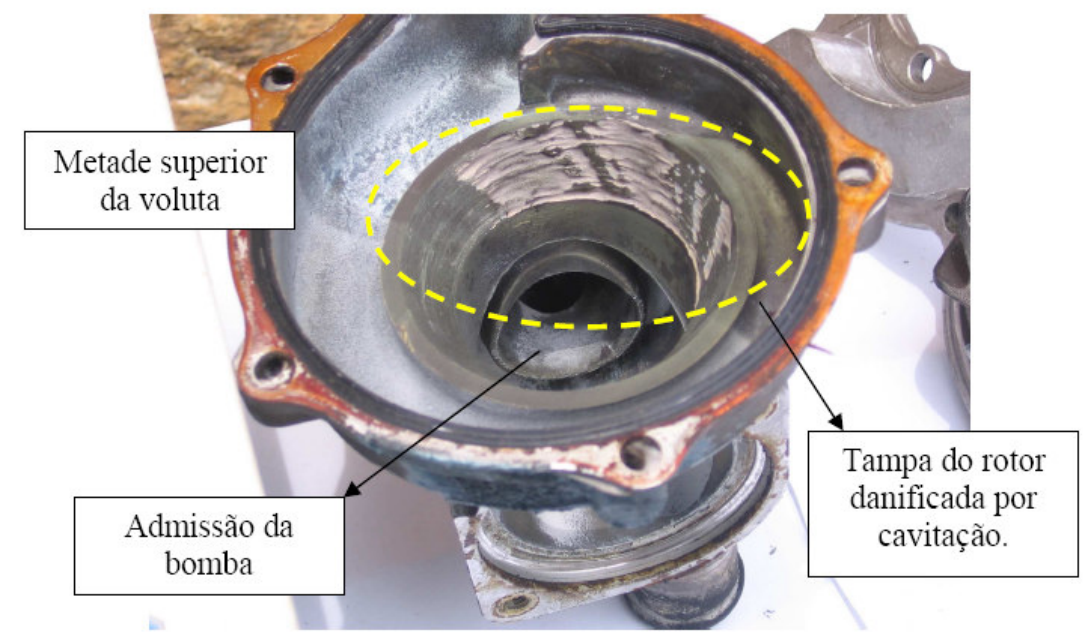

Figura 6 - Capa do rotor danificada por cavitação. Imagem gentilmente cedida por MARIANI, A. L. C.

A Figura 6 indica a parte superior da voluta de uma bomba de um motor de combustão interna, cuja tampa do rotor sofreu danos por cavitação. Logo acima encontra-se a admissão da bomba. A Figura 7 mostra as duas metades da bomba cavitada, de forma a propiciar um melhor entendimento da localização dos danos.

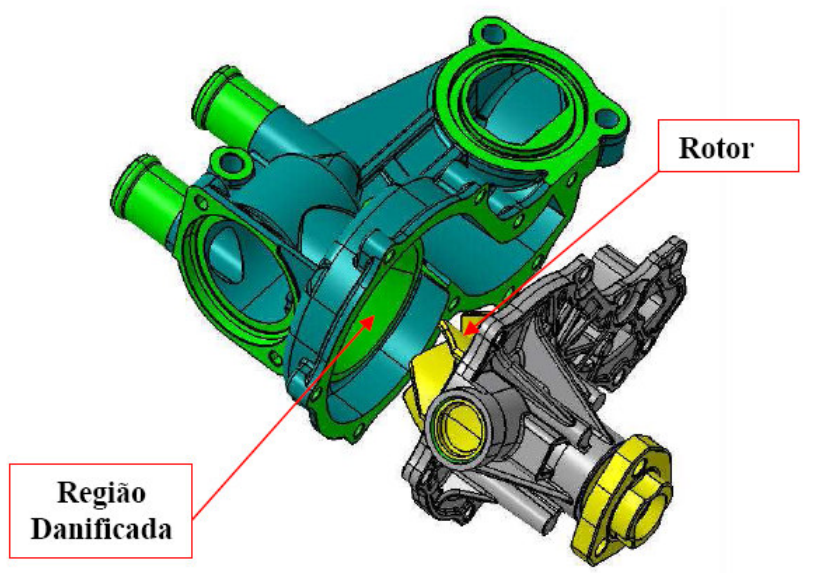

Figura 7 - Condição de Montagem da Bomba Danificada 
A pressão de impacto gerada pelo colapso de bolhas de cavitação tem sido alvo de inúmeros estudos. A figura 8 apresenta o resultado do trabalho de TOMITA, SHIMA, TAKAYAMA E OHSAWA (1983), onde uma série de fotografias do colapsar de uma bolha de cavitação ao longo da correspondente pressão na superfície sólida próxima da bolha são correlacionados. As medições indicam que as maiores pressões se deram quando a bolha atingiu seu menor volume. Este instante ocorre entre as imagens 6 e 7 .
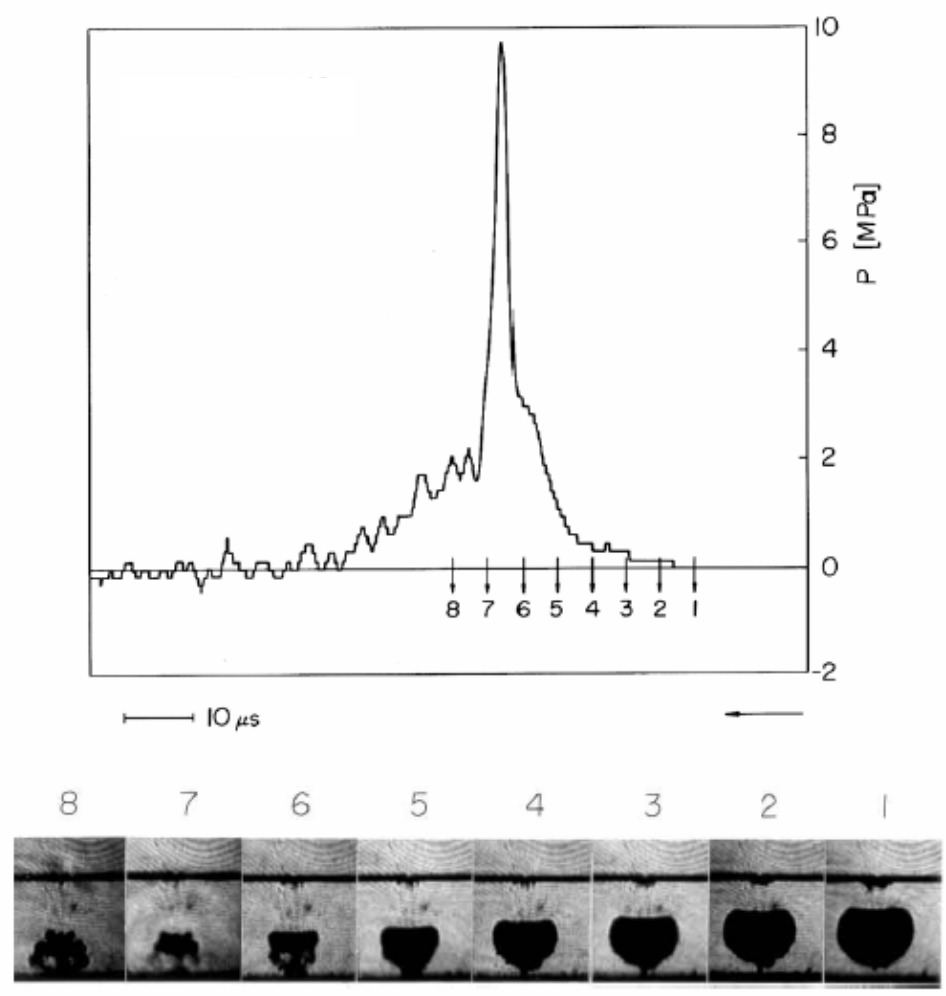

Figura 8 - Monitoramento de pressões ao longo do colapso de uma bolha de cavitação

Segundo IWAI (2000), a resistência à erosão por cavitação não apresenta uma boa correlação com uma única propriedade mecânica, tal como tensão de ruptura ou dureza. Entretanto, uma correlação muito melhor pode ser obtida com a resiliência máxima, 
$R m=\frac{1}{2} \frac{\sigma r^{2}}{E}$

onde:

$R m$ - resiliência máxima;

or - tensão de ruptura;

$E$ - modulo de elasticidade,

ou pelo trabalho realizado para causar a fratura,

$\omega r=\sigma \frac{2}{3}(\sigma r-\sigma) L$

onde:

$\omega r$ - trabalho feito para causar a fratura;

$\sigma$ - limite de elasticidade;

or - tensão de ruptura;

$L$ - deformação.

Porém, ainda segundo Iwai (2000), nenhum relacionamento unificado, que se repete sem exceções para uma vasta gama de materiais foi encontrado até agora. Presume o autor que isso se deve ao fato de que o colapso de uma bolha de cavitação age em uma área muito pequena, o que faz alguns materiais desviarem de correlações de propriedades mecânicas que são determinadas através de avaliações de corpos de prova que envolvem uma porção maior do material. Um exemplo disso seria o ferro fundido, que apresenta uma resistência micro-escalar menor do que a encontrada quando se avalia um corpo de prova. Em adição, materiais como os aços inoxidáveis desviam das correlações porque a micro-estrutura de sua superfície muda devido à ação repetida de pequenos impactos. 
A ocorrência de cavitação não implica necessariamente na ocorrência de erosão. A detecção do exato momento em que a erosão se inicia ainda é imprevisível devido às complexidades das condições de escoamento e da inabilidade de se analisar ou modelar essas condições.

O índice de 3\% de diminuição da altura manométrica é normalizado e entendido como limite de trabalho pela grande maioria dos fabricantes de bombas, mesmo se sabendo que a cavitação se inicia antes desse ponto. Evitar a cavitação completamente torna-se muitas vezes impraticável, devido ao fato do dimensionamento da entrada da bomba ser limitado ao espaço disponível,

Chan (1988) questiona o índice de 3\%. Ele conduziu experimentos e mostrou que abaixo de um NPSH critico (o termo NPSH será detalhado logo abaixo), a taxa de erosão aumenta, para depois diminuir, e então aumentar definitivamente para um NPSH decrescente. $\mathrm{O}$ experimento foi conduzido através da pintura e traçagem de uma malha de $5 \mathrm{~mm} \times 5 \mathrm{~mm}$ nas superfícies das pás de um rotor, de forma que a região atacada pela cavitação fosse facilmente identificada. Para uma dada vazão, o NPSH foi diminuído de forma que a cavitação se iniciasse, seguido da queda de $3 \%$ na altura manométrica. $\mathrm{O}$ método estabelece que quando a cavitação se inicia a um determinado NPSH, a taxa de erosão é zero, e quando o NPSH é diminuído abaixo deste ponto, a taxa de erosão aumenta, como é representado na Figura 9.

Os resultados encontrados por Chan são expressos na Figura 10, onde é possível visualizar a existência de uma taxa máxima e uma taxa mínima de erosão entre os pontos de inicio de cavitação e o de redução de $3 \%$ na altura manométrica. 


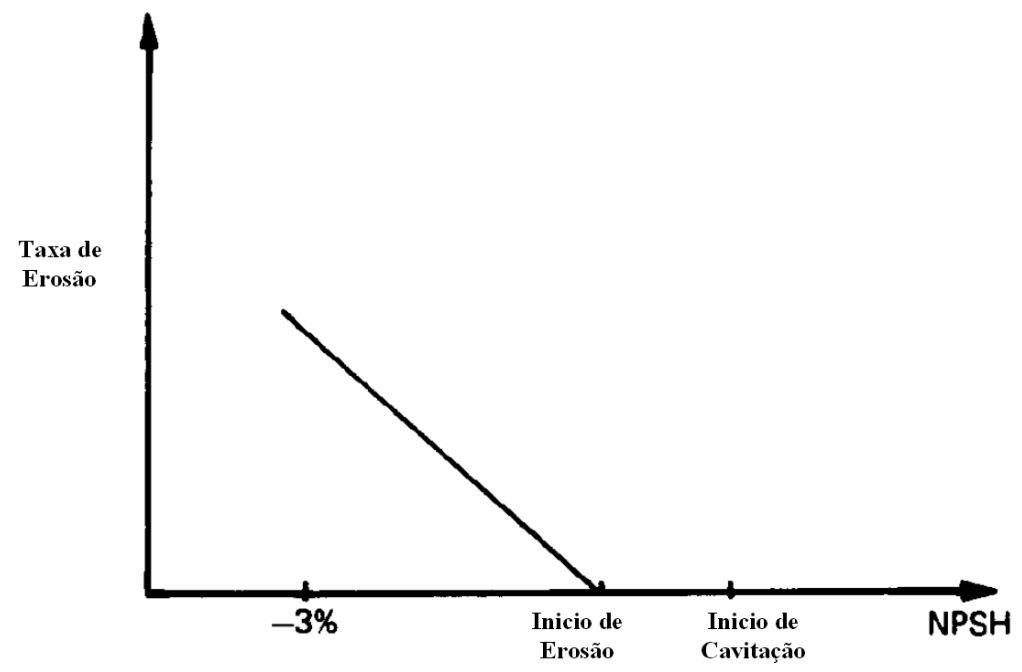

Figura 9 - Taxa Teórica de Erosão x NPSH

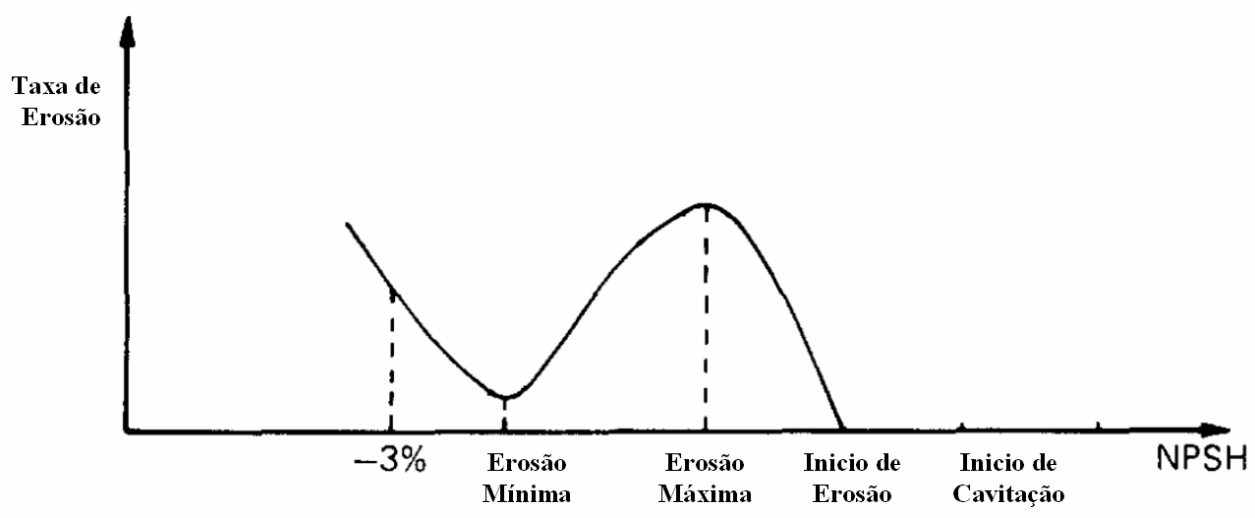

Figura 10 - Taxa de Erosão x NPSH, Chan (1988)

Brennen (1994) detalha os parâmetros de cavitação da seguinte forma:

A pressão estática $(p)$ é normalmente expressa por um número adimensional chamado de coeficiente de pressão $(C p)$, definido como

$C_{p}=\left(p-p_{1}\right) / \frac{1}{2} \rho U^{2}$ 
onde $p_{1}$ é pressão na entrada da bomba e $U$ a velocidade na ponta da pá na aresta de sucção dado por $\Omega R_{T 1}$ onde $\Omega$ é a velocidade angular e $R_{T 1}$ é o raio da aresta de sucção. $\mathrm{Na}$ ausência de cavitação a velocidade do fluido e o coeficiente de pressão são independentes do nível de pressão. Por exemplo, uma mudança na pressão de entrada $\left(p_{1}\right)$, irá simplesmente resultar em uma mudança igual em todas as outras pressões, não afetando $C_{p}$. Dessa forma, para qualquer escoamento haverá uma localização particular para a qual a pressão $p$ é mínima e a diferença entre essa pressão mínima $\left(p_{\min }\right)$ e a pressão de entrada $\left(p_{1}\right)$ será dada por:

$$
C_{p \min }=\left(p_{\min }-p_{1}\right) / \frac{1}{2} \rho U^{2}
$$

Ainda segundo Brennen (1994), se o valor de $C_{p \min }$ puder ser obtido de forma teórica ou experimental, então é possível estabelecer o valor para a pressão de entrada $\left(p_{1}\right)$ para a qual a cavitação se iniciaria, admitindo-se que isso ocorre quando $p_{\min }=$ pressão de vapor $\left(p_{v}\right)$.

$$
\left(p_{1}\right)_{\substack{\text { Inicioda } \\ \text { cavitaça }}}=p_{v}+\frac{1}{2} \rho U^{2}\left(-C_{p \min }\right)
$$

Muitos parâmetros adimensionais são usados para avaliar o potencial para a ocorrência de cavitação. Um dos mais fundamentais seria o Número de Cavitação $(\sigma)$, definido por:

$$
\sigma=\left(p_{1}-p_{v}\right) / \frac{1}{2} \rho U^{2}
$$

Claramente todo escoamento tem um valor para o número de cavitação ocorrendo ou não cavitação. Existe porém, um valor especifico para o número de cavitação correspondente a uma pressão de entrada particular 
$\left(p_{1}\right)$ para a qual a cavitação ocorre conforme a pressão é diminuída. Esse valor é chamado de número de inicio de cavitação, denotado por $\sigma_{i}$ :

$$
\sigma_{i}=\left[\left(p_{1}\right)_{\substack{\text { Inicioda } \\ \text { cavitaşão }}}-p_{v}\right] / \frac{1}{2} \rho U^{2}
$$

Se o inicio da cavitação ocorre quando $p_{\min }=p_{v}$, então combinando as equações (3) e (5), fica claro que por esse critério o número de inicio de cavitação corresponde a $\sigma_{i}=-C_{p \min }$.

Outros parâmetros de cavitação são freqüentemente utilizados. O NPSP (Net Positive Suction Pressure) é o acrônimo para $\left(p_{1}^{T}-p_{V}\right)$, onde $p^{T}{ }_{1}$ é a pressão total na entrada, dado por:

$$
p_{1}^{T}=p_{1}+\frac{1}{2} \rho v_{1}^{2}
$$

O NPSE (Net Positive Suction Energy) é definido por $\left(p_{1}^{T}-p_{V}\right) / \rho$, e o NPSH (Net Positive Suction Head) por $\left(p_{1}^{T}-p_{V}\right) / \rho g$, sendo esse ultimo um dos mais utilizados.

\subsection{Efeitos da Mistura Água e Etilenoglicol, Temperatura do Fluido e Pressão do Sistema}

Hattori (2006) estuda a influência da temperatura da água na erosão por cavitação. Ele conduziu testes de erosão a várias temperaturas, mantendo o número de cavitação constante através da compensação da pressão à medida que a temperatura aumentava. O corpo de prova foi pesado a cada 10 minutos e o teste continuou até que a taxa de erosão chegasse ao máximo, para então começar a cair. 
A Figura 11 mostra a curva determinada por Hattori ao utilizar um corpo de prova de cobre puro, mantendo um número de cavitação de $\sigma=0.015$.

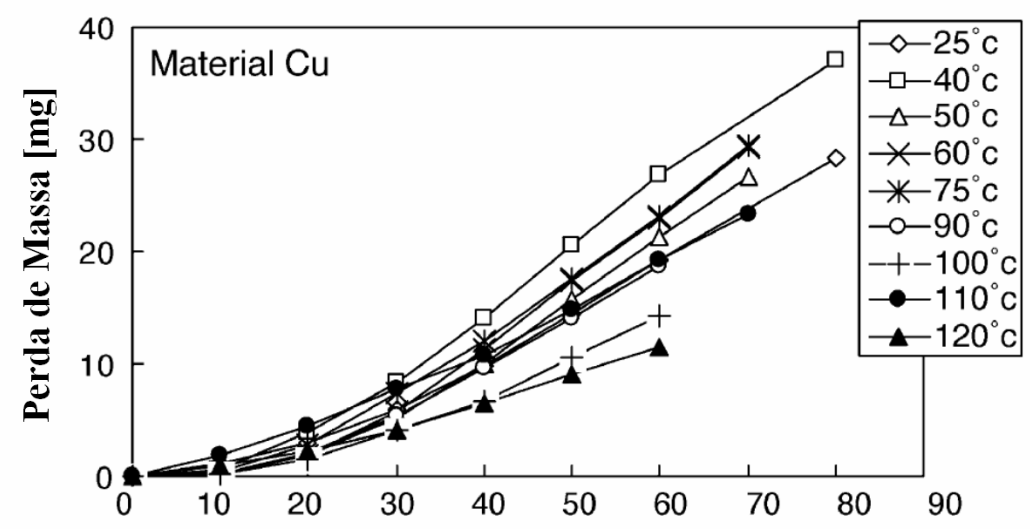

Tempo de Exposição [min]

Figura 11 - Curva de Perda de Massa ( $\sigma=0.015$ ) - Hattori (2006).

Em sua avaliação Hattori considera um parâmetro chamado MDER (Mean Deph of Erosion Rate ou Profundidade Média da Taxa de Erosão), definido pela perda de massa dividida pela massa especifica do material, área erodida e intervalo de tempo de exposição.

As Figuras 12 (a) e (b) mostram os resultados para os números de cavitação $\sigma=0,015$ e 0,02 . Para $\sigma=0,015$, as curvas de MDER mostram um aumento gradual. Após alcançar o pico em torno de $45 \mathrm{~min}$, O MDER parece estabilizar. 


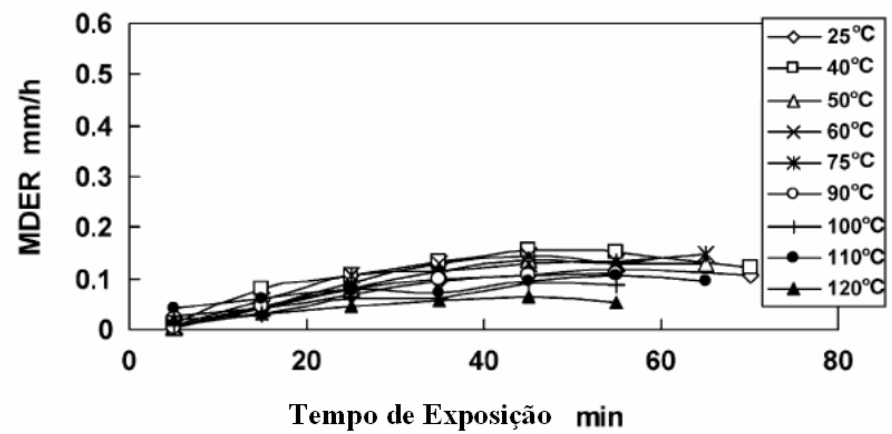

(a) $\sigma=0.015$

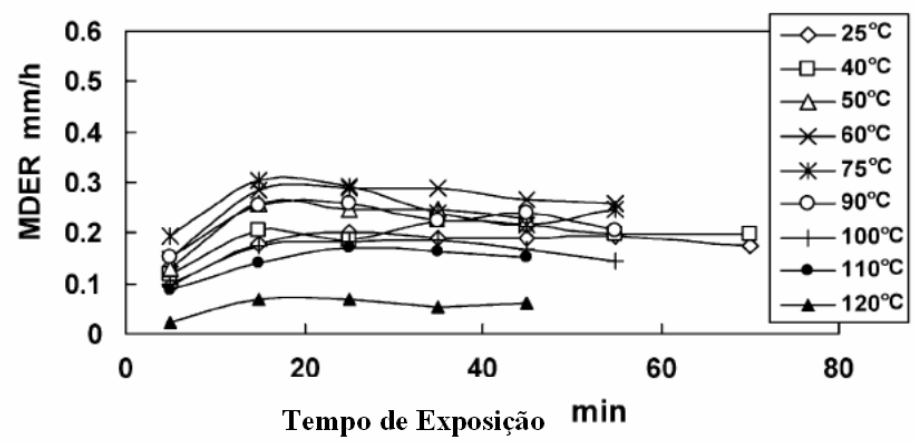

(b) $\sigma=0.02$

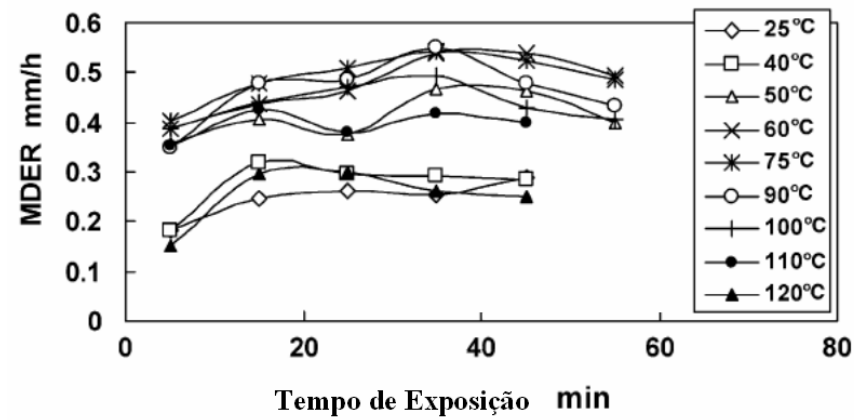

(c) $\sigma=0.025$

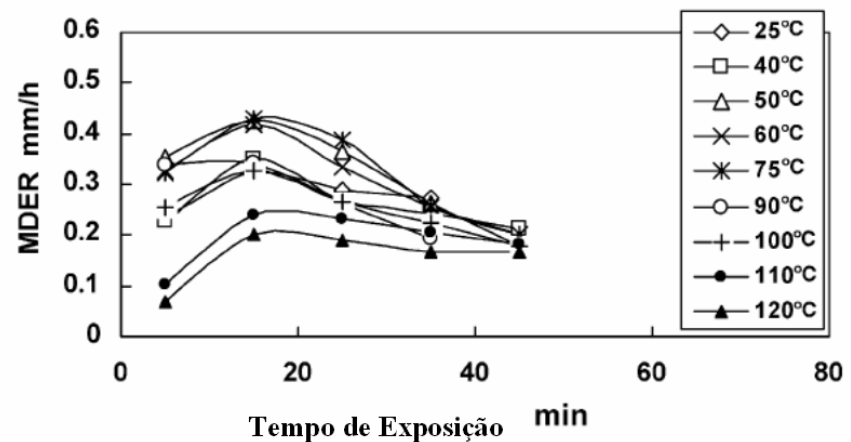

(a) $\sigma=0.03$

Figura 12 - Curvas de MDER - Hattori (2006). 
Para $\sigma=0,020$, a área erodida é próxima daquela encontrada para $\sigma=0,015$, entretanto o tempo para alcançar o pico é menor, em torno de 15 min. O tempo para alcançar o pico em $40^{\circ} \mathrm{C}$ é prolongado para algo próximo de 45 min. O MDER a $120^{\circ} \mathrm{C}$ é muito menor que o encontrado a $110^{\circ} \mathrm{C}$.

As Figuras 12 (c) e (d) mostram as curvas de MDER para os números de cavitação $\sigma=0,025$ e 0,030 , respectivamente. Para $\sigma=0,025$, o MDER alcança a taxa máxima após um período de $15 \mathrm{~min}$. Apesar de a curva apresentar flutuações, ela permanece constante até o período de $60 \mathrm{~min}$. O MDER aumenta com a temperatura do liquido até $90{ }^{\circ} \mathrm{C}$, e diminui quando acima de $100^{\circ} \mathrm{C}$.

Para $\sigma=0,030$, o MDER alcança o pico em $15 \mathrm{~min}$, seguido de uma diminuição. O MDER aumenta com temperaturas do liquido entre 50 e $75^{\circ} \mathrm{C}$, mas diminuí para temperaturas maiores. O MDER máximo foi definido como o valor máximo de uma curva de MDER mostrado na Figura 16.

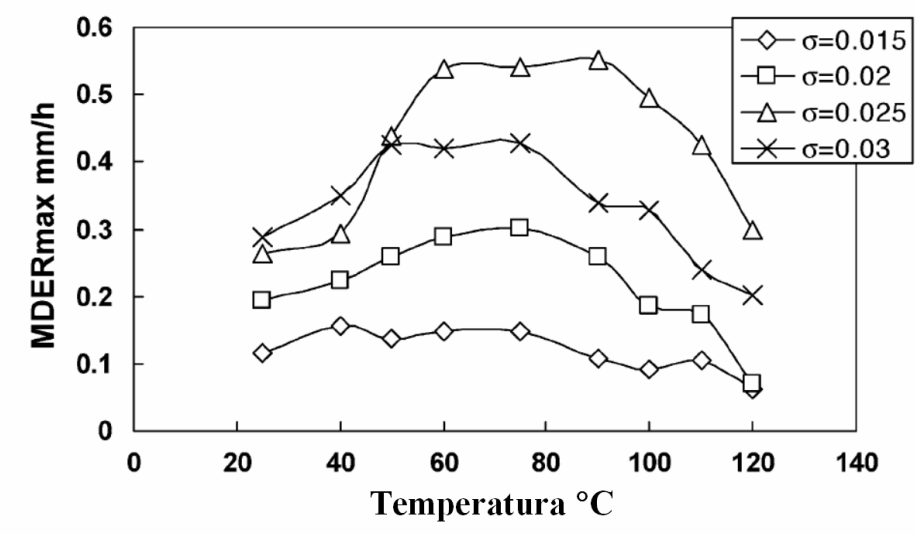

Figura 13 - Relação entre Temperatura e MDER máximo - Hattori (2006).

A Figura 13 mostra a relação entre a temperatura do líquido e o $M D E R_{\max }$. $O$ MDER max aumenta gradativamente com a temperatura, e alcança um pico a $75{ }^{\circ} \mathrm{C}$, seguido de um decréscimo gradual. $\mathrm{O}$ comportamento do MDER $R_{\max }$ 
para $\sigma=0,020$ é praticamente o mesmo que para $\sigma=0,015$, porém o valor para $\sigma=0,020$ é próximo do dobro do valor para $\sigma=0,015$. O pico de valor para $75{ }^{\circ} \mathrm{C}$ é 1.6 vezes maior que para $25{ }^{\circ} \mathrm{C}$. O comportamento do MDER $_{\text {max }}$ para $\sigma=0,025$, mostra uma tendência similar ao $\sigma=0,020$. O pico do $\mathrm{MDER}_{\max }$ muda para a temperatura de $90^{\circ} \mathrm{C}$. O pico para $\sigma=0,025$ é em torno de 1.8 vezes maior que para $\sigma=0,020$. O pico para $\sigma=0,030$ volta a cair para $75^{\circ} \mathrm{C}$, e é 0,78 vezes menor que para $\sigma=0,025$.

Hosny (2006), através de um método de monitoramento das emissões acústicas resultantes das implosões das bolhas durante o processo de cavitação, encontra um resultado similar ao de Hattori, ao avaliar a intensidade de cavitação, medida em dB, em função da temperatura. Hosny conduz o experimento utilizando água como fluido de teste, e seus resultados são mostrados na Figura 14.

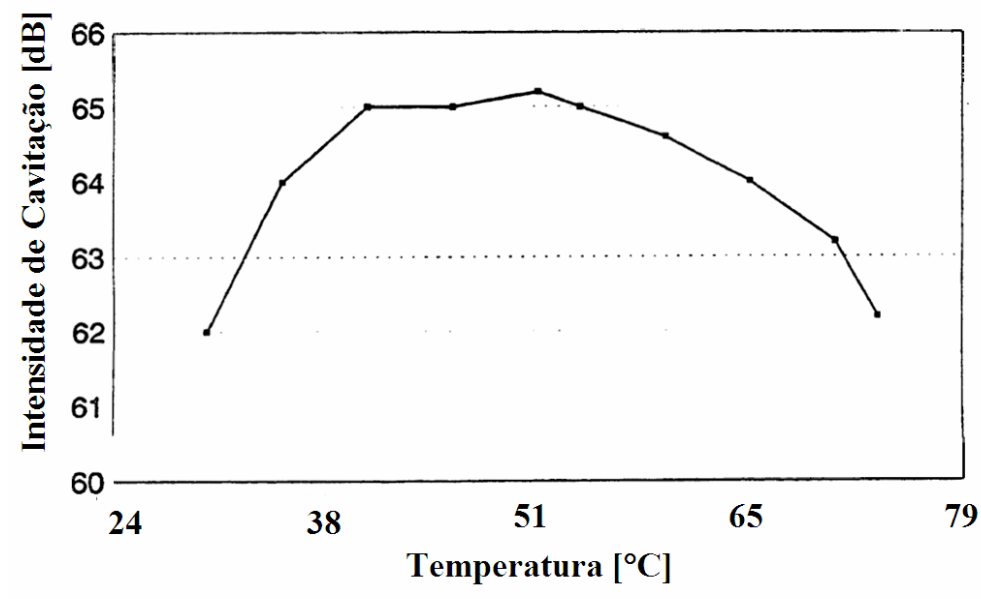

\section{Figura 14 - Intensidade de Cavitação em Função da Temperatura - Hosny (2006).}

Hosny (2006) estuda também a intensidade de cavitação e avalia a perda em massa de material, utilizando um corpo de prova de ferro fundido ao se 
trabalhar com condições iguais ás de cavitação, porém com diferentes concentrações da mistura água/etilenoglicol. Seus resultados são apresentados nas Figuras 15 e 16.

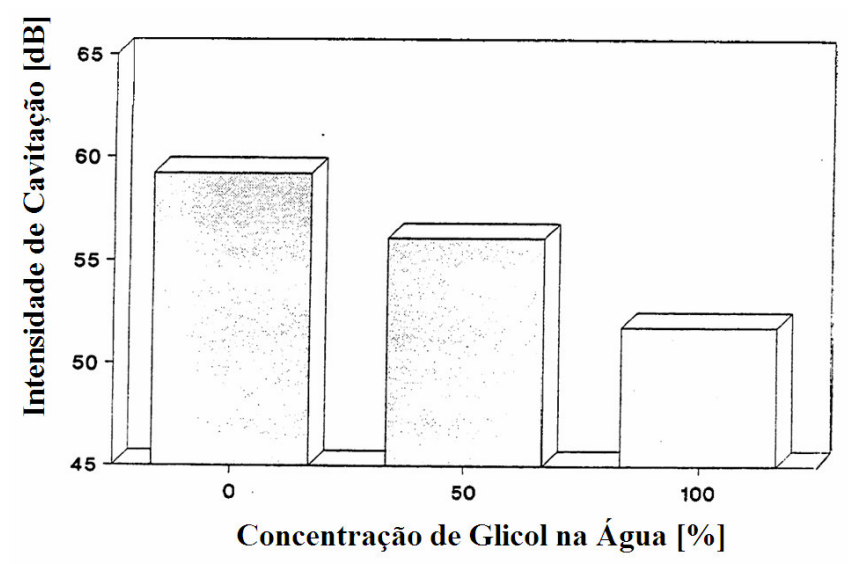

Figura 15- Intensidade de Cavitação em Função da Concentração de Etilenoglicol - Hosny (2006).

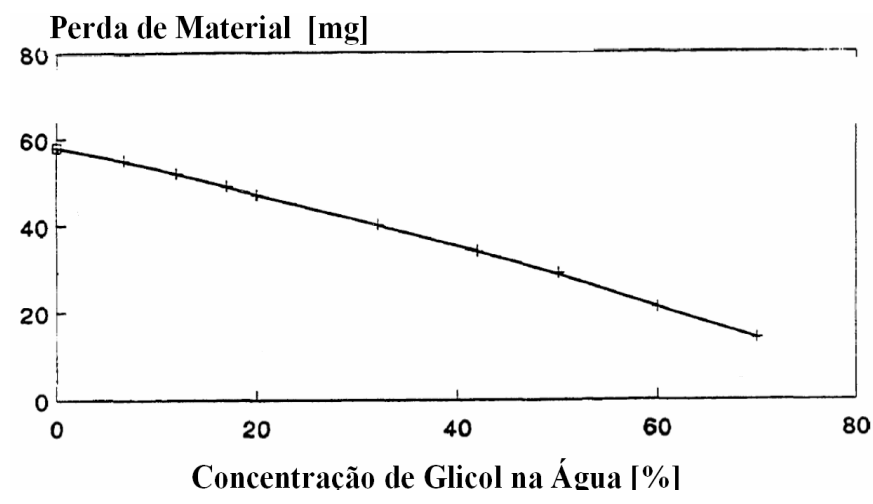

Figura 16 - Efeito da Concentração de Glicol na Erosão por Cavitação Hosny (2006).

Com o objetivo de estudar os critérios para a ocorrência e prevenção de cavitação em bombas d'água aplicadas no mercado automotivo, Kim (2000) realiza em bancada observações do fenômeno de cavitação ao se utilizar como fluido de arrefecimento, uma mistura de 50\% água e 50\% etilenoglicol. O teste foi conduzido controlando e avaliando varias pressões de sistema e temperaturas do fluido. $O$ estudo mostra a dependência desses dois fatores 
na ocorrência de cavitação e avalia o efeito da cavitação sobre a eficiência e o desempenho da bomba.

A temperatura de cavitação em uma bomba d'água aplicada no sistema de arrefecimento de um motor de combustão interna é estudada por Huang (2004), usando três fluidos diferentes: 100\% água, 50\% etilenoglicol e 50\% água, e 100\% etilenoglicol, em diversas rotações de trabalho. Seus testes concluem que apesar da excelente condutividade térmica, a água é muito mais propensa à formação de bolhas de vapor devido ao fato de ter um ponto de ebulição menor que o etilenoglicol. Com isso a temperatura de cavitação da bomba d'água trabalhando com esse fluido é relativamente baixa. Em contraste, o fluido de arrefecimento contendo $100 \%$ etilenoglicol tem uma condutividade térmica menor, mas é menos suscetível à formação de bolhas de vapor devido a seu maior ponto de ebulição. Dessa forma, tanto o ponto de ebulição, como a capacidade de prevenir cavitação aumentam com o percentual de etilenoglicol. Neste artigo, também se conclui que a mistura 50\% água e 50\% etilenoglicol tem um comportamento similar a $100 \%$ água, de forma que a temperatura de cavitação aumenta somente quando o percentual de etilenoglicol excede $50 \%$. O autor entende que a pressão do sistema é um dos principais fatores para a ocorrência de cavitação, porém ele não controla esse parâmetro em seu experimento, de forma que a mesma varia ao longo do teste em função das mudanças de temperatura.

Pode-se observar que diversos trabalhos já foram desenvolvidos visando estudar, de forma ampla ou específica, o comportamento e os efeitos do fenômeno de cavitação. Alguns trabalhos se dedicam ao estudo da influência da temperatura e da pressão do sistema na geração de cavitação, além de avaliar a sua influência sobre a eficiência e desempenho da bomba. Outros trabalhos têm como objetivo principal estudar o efeito de diferentes misturas de água e etilenoglicol no fenômeno de cavitação, ao se trabalhar em variadas temperaturas. Esses estudos avaliam os três fatores de maior 
influência no controle da cavitação em sistemas de arrefecimento. Estes fatores são a pressão de vapor do fluido, a pressão e a temperatura do sistema de arrefecimento. Entretanto, nenhum dos trabalhos encontrados apresenta resultados de ensaios onde esses três parâmetros são controlados simultaneamente. 


\section{AVALIAÇÃO EXPERIMENTAL DA INFLUÊNCIA DA CONCENTRAÇÃO DE ETILENOGLICOL NA TEMPERATURA DE CAVITAÇÃO}

\subsection{Medição dos Parâmetros de Trabalho do Sistema de Arrefecimento}

Com o intuito de analisar o comportamento e as variações de temperatura e pressão do sistema de arrefecimento, um veículo equipado com um motor $1,8 \mathrm{~L}$ e que utiliza gasolina como combustível foi instrumentado de acordo com o esquema da Figura 17.

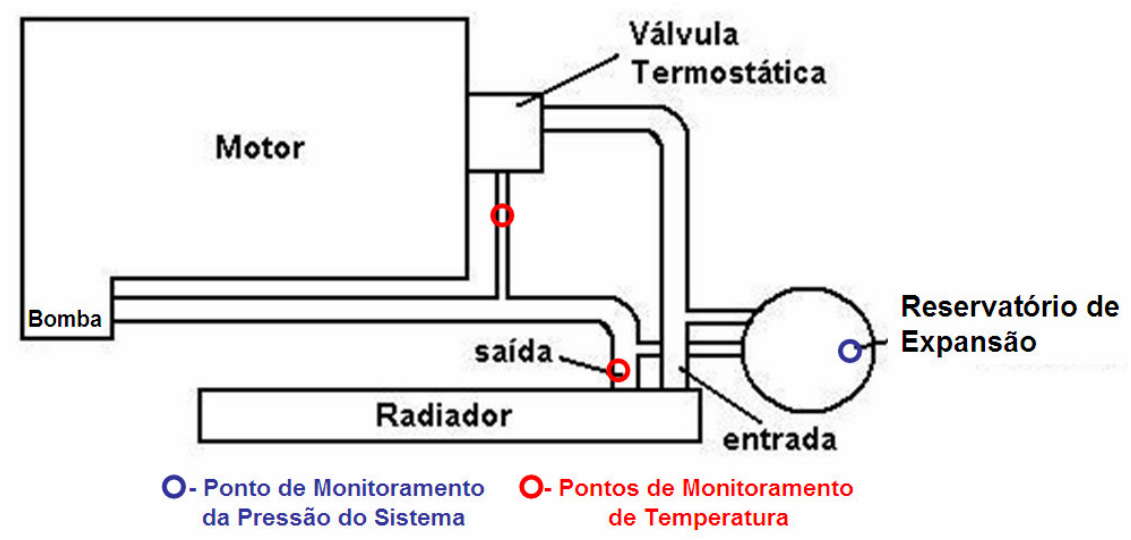

Figura 17 - Instrumentação do Motor

A válvula termostática tem a função de prover um aquecimento rápido do motor e de controlar a sua temperatura, fazendo com que o fluido de arrefecimento não circule pelo radiador até atingir uma temperatura em torno de $90{ }^{\circ} \mathrm{C}$. Como o sistema é fechado, sua pressão varia em função da variação de sua temperatura. 
Para a medição das temperaturas e pressões do sistema foram utilizados os seguintes equipamentos:

- Termopares tipo K, que operam em uma faixa de -200 a $1200{ }^{\circ} \mathrm{C}$, com uma exatidão de 99,6 \%, fabricados pela empresa lope Instrumentos de precisão Ltda. As Figuras 18 e 19 indicam respectivamente os termopares montados no by-pass da válvula termostática e na saída do radiador.

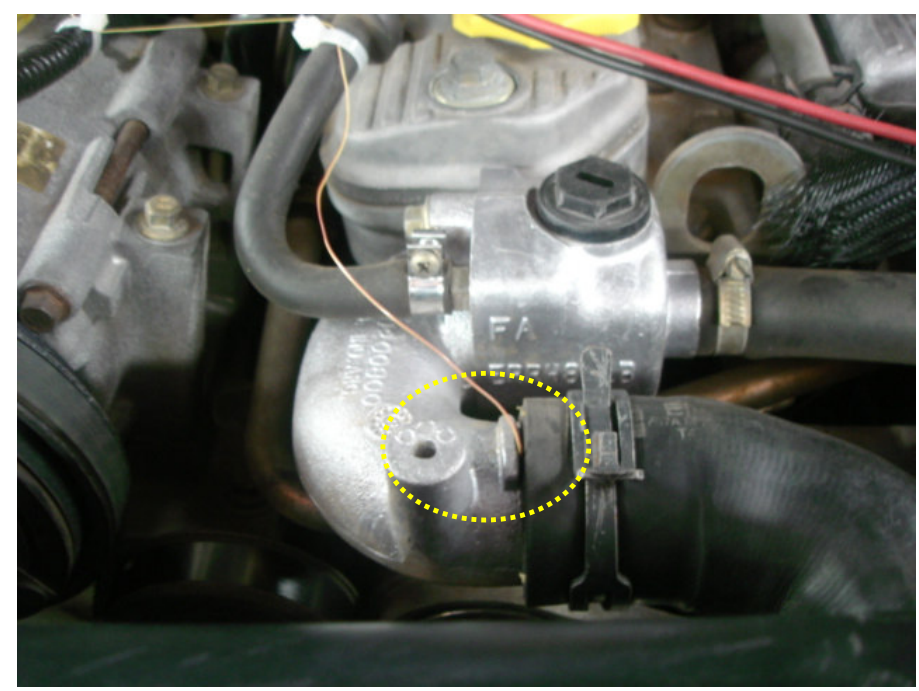

Figura 18 - Termopar Montado no By-Pass da Válvula Termostática

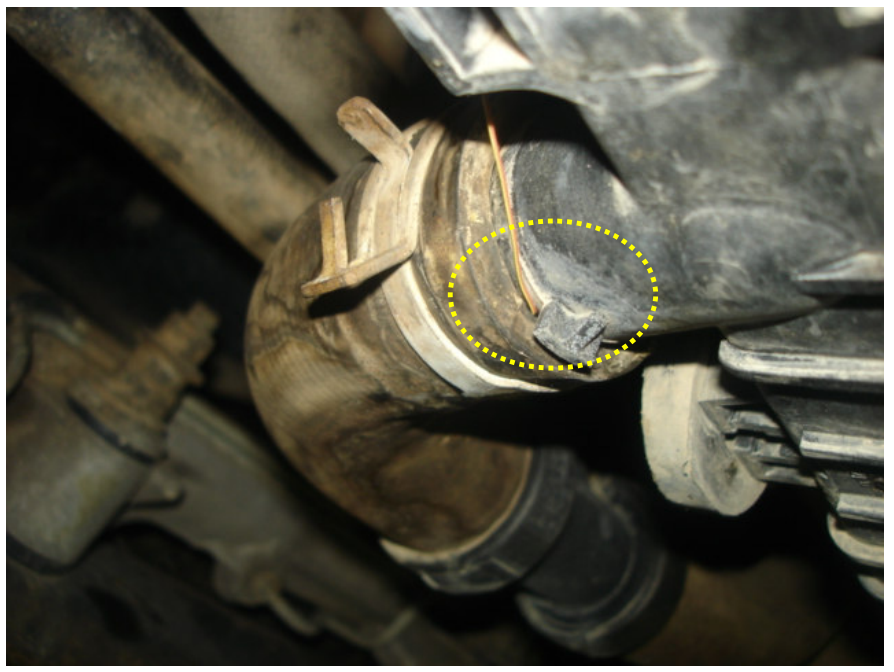

Figura 19 - Termopar Montado na Saída do Radiador 
- Transdutores de pressão piezoelétricos, com faixa de trabalho de -1 a 20 bar, exatidão de 99,7 \%, fabricados pela empresa Novus Eletronics. A Figura 20 ilustra o transdutor utilizado;

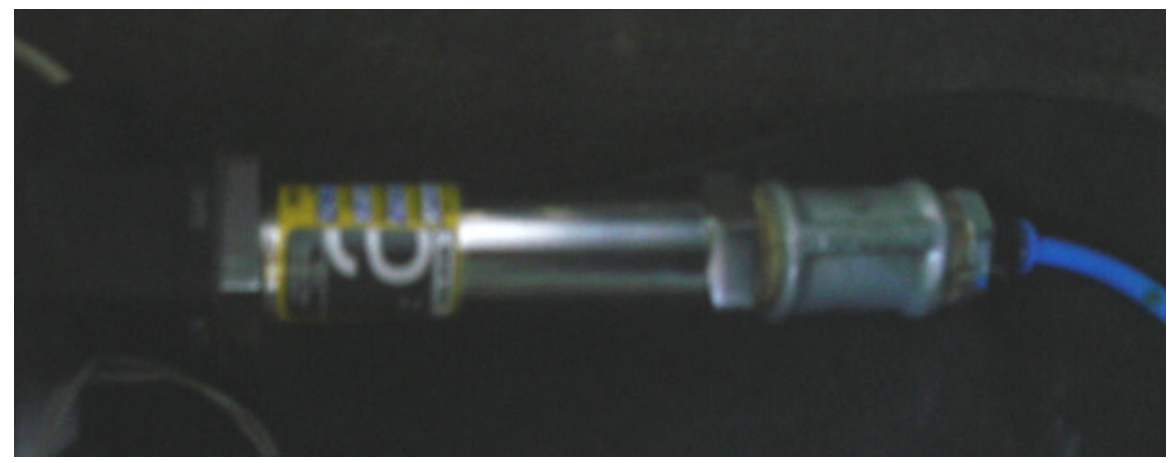

Figura 20 - Transdutor de Pressão

- Módulo FP-Al-110, fabricado pela National Instruments, que mede os sinais de 4 a $20 \mathrm{~mA}$ enviados pelo transdutor de pressão e retorna valores escalares para o software de monitoramento. Módulo FP-TC120, fabricado pela National Instruments, específico para receber sinais de termopares, e retorná-los como valores escalares para o software de monitoramento. A Figura 21 ilustra esses equipamentos.

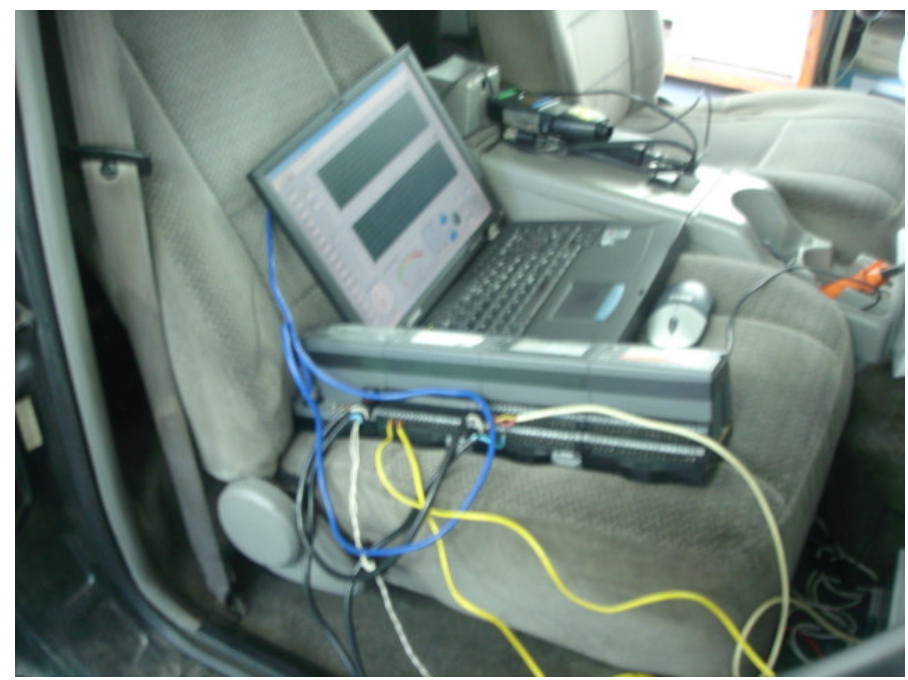

Figura 21 - Módulos para Conversão dos Sinais 
- Software de monitoramento LabView 7.1, desenvolvido pela National Instruments, que recebe, trata e apresenta os resultados.

A Figura 22 identifica com um circulo tracejado o ponto onde a pressão do sistema foi medida.

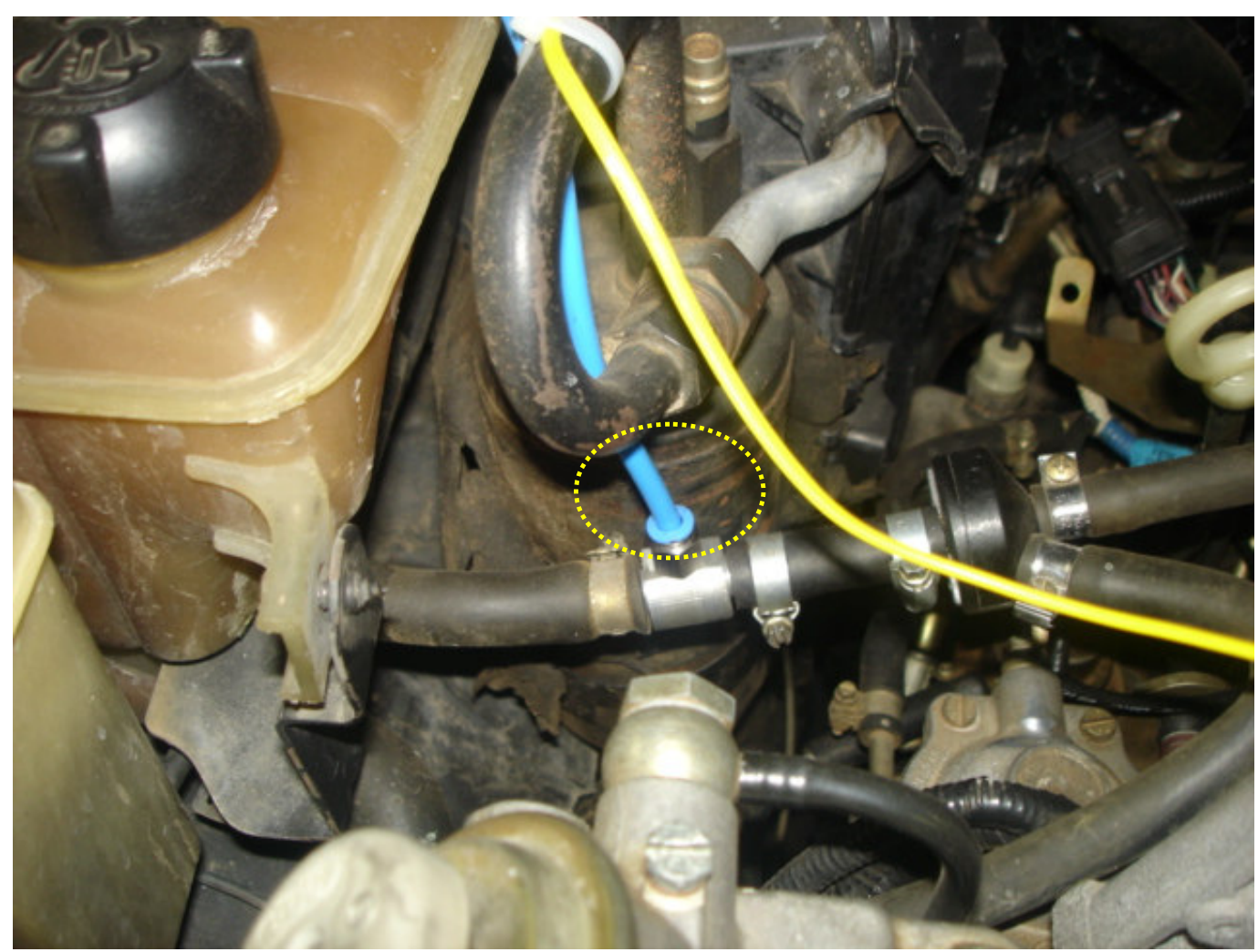

Figura 22 - Ponto de Tomada das Pressões do Sistema

A Figura 23 apresenta o resultado do monitoramento do veículo ao trafegar em condições variadas de estrada e cidade. As informações foram armazenadas a cada 5 segundos. 


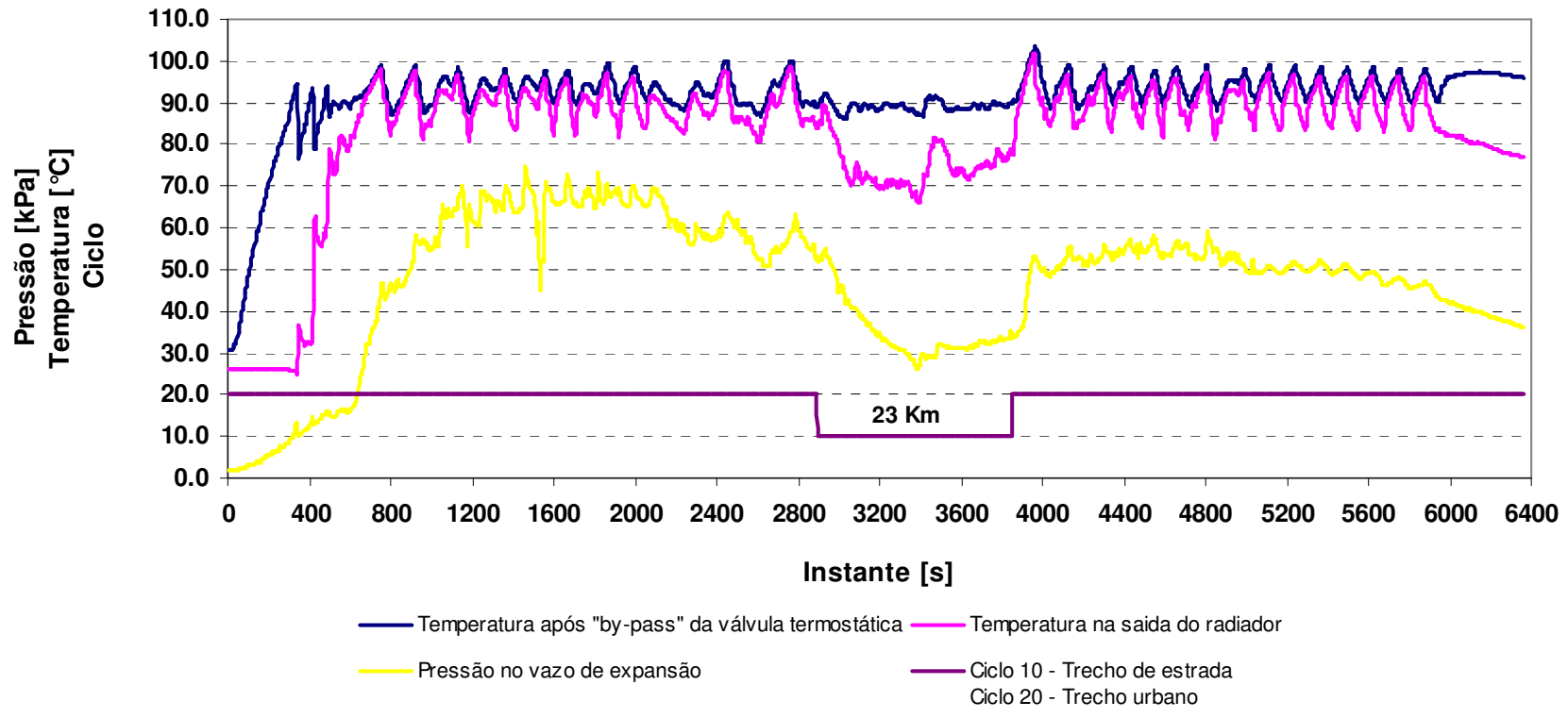

Figura 23 - Monitoramento de Pressões e Temperaturas do Sistema de Arrefecimento

As medições se iniciam poucos instantes após a partida do motor, quando a temperatura na saída da válvula termostática está em torno de $30{ }^{\circ} \mathrm{C}$, a temperatura na saída do radiador $26{ }^{\circ} \mathrm{C}$, e a pressão no reservatório de expansão é igual à atmosférica.

A temperatura na saída da válvula termostática aumenta continuamente até o instante $345 \mathrm{~s}$, quando a válvula se abre a $94^{\circ} \mathrm{C}$, liberando a passagem do fluido de arrefecimento.

A partir deste ponto a válvula passa a atuar abrindo e fechando a passagem para o radiador. No instante $757 \mathrm{~s}$ a temperatura na saída da válvula termostática se aproxima da temperatura do fluido de arrefecimento na saída do radiador e mantém um comportamento similar para o primeiro trecho de cidade, que corresponde ao instante 0 e 2900 , onde a temperatura no by pass da válvula termostática oscila por volta de 90 e $100{ }^{\circ} \mathrm{C}$, enquanto a temperatura na saída do radiador se alterna entre 80 e $100^{\circ} \mathrm{C}$. 
Até este ponto, a condição mais critica em termos de ocorrência de cavitação se dá no instante 342 s, ainda na fase de aquecimento do motor, quando a temperatura do fluido de arrefecimento chega a $94{ }^{\circ} \mathrm{C}$ e a pressão no sistema é de apenas $11 \mathrm{kPa}$.

A avaliação dos parâmetros de trabalho em estrada é feita em um percurso de 23 km, compreendido entre os instantes 757 e 3862 s. Para esta condição, a temperatura na saída do radiador se alterna entre 70 e $80^{\circ} \mathrm{C}$, aproximadamente, enquanto a temperatura na saída da válvula termostática oscila entre 85 e $90^{\circ} \mathrm{C}$, sendo que a menor pressão de sistema encontrada foi de $26 \mathrm{kPa}$.

Ao retornar ao perímetro urbano, monitora-se um pico de temperatura onde o fluido de arrefecimento, na saída do radiador e da válvula termostática, chega a $103^{\circ} \mathrm{C}$ no instante 3967 s, quando a pressão de sistema é $53 \mathrm{kPa}$. 


\subsection{Bancada de Teste}

A bancada de teste ilustrada na Figura 24 é constituída de:

a) um reservatório de aço inoxidável ligado a uma linha de ar comprimido, uma linha de água refrigerada e resistências elétricas, que têm respectivamente a função de pressurizar o sistema e controlar a temperatura do fluido de teste. $O$ reservatório tem capacidade total de 80 litros, e opera com $75 \%$ de sua capacidade ocupada com liquido de arrefecimento e os outros $25 \%$ com ar pressurizado. A proporção é controlada através do indicador de nível.
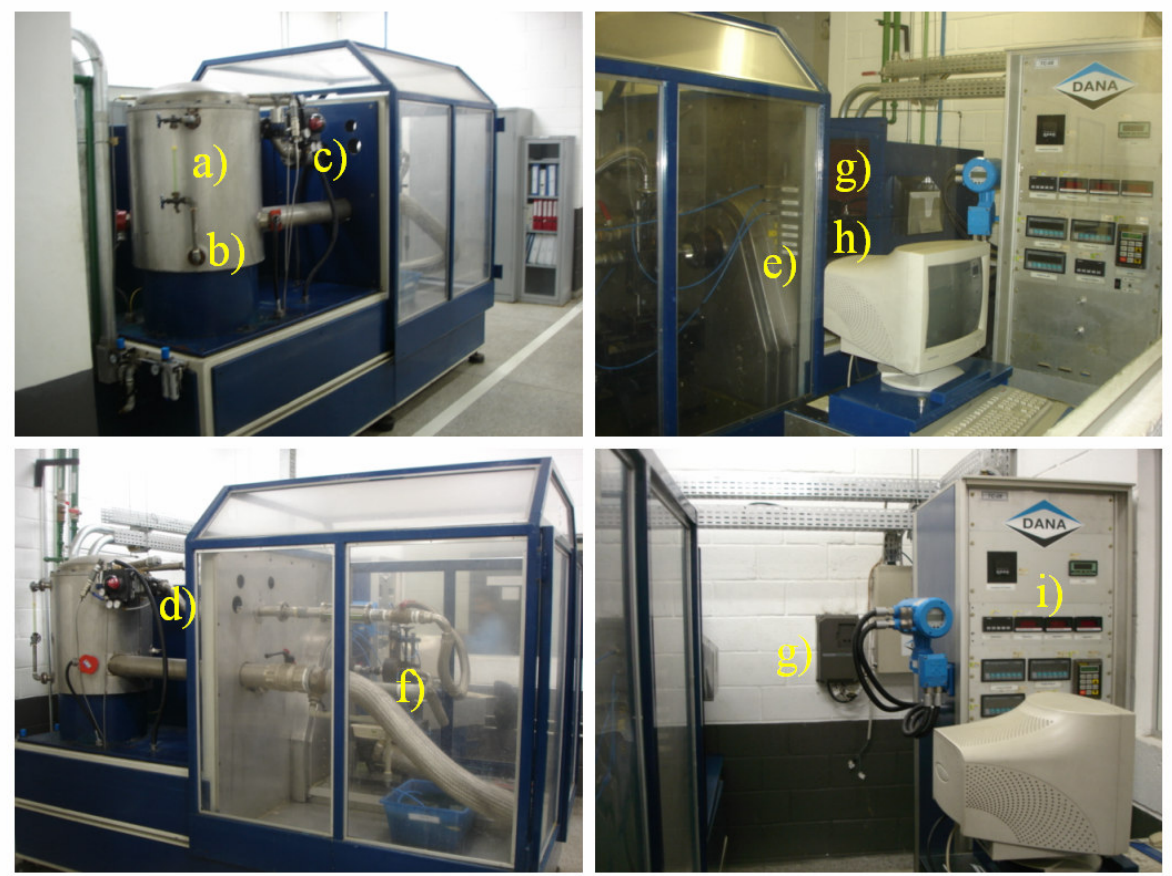

Figura 24 - Bancada de Teste

b) As resistências elétricas são flangeadas e construídas com elementos tubulares blindados de aço inoxidável, isolados internamente com óxido de 
magnésio, com o filamento resistivo feito de $\mathrm{Nl}-\mathrm{Cr} 80 / 20$, encapsulados em tubos de aço inoxidável. Cada resistência tem uma potência de $450 \mathrm{~W}$, e são alimentadas com corrente alternada a uma tensão de $230 \mathrm{~V}$ e $60 \mathrm{~Hz}$.

c) Uma válvula proporcional eletro-pneumática, posicionada após o medidor de vazão, como ilustrado na Figura 25 , tem como sinal de entrada $4 \mathrm{~mA}$ (totalmente aberta) a $20 \mathrm{~mA}$ (totalmente fechada).

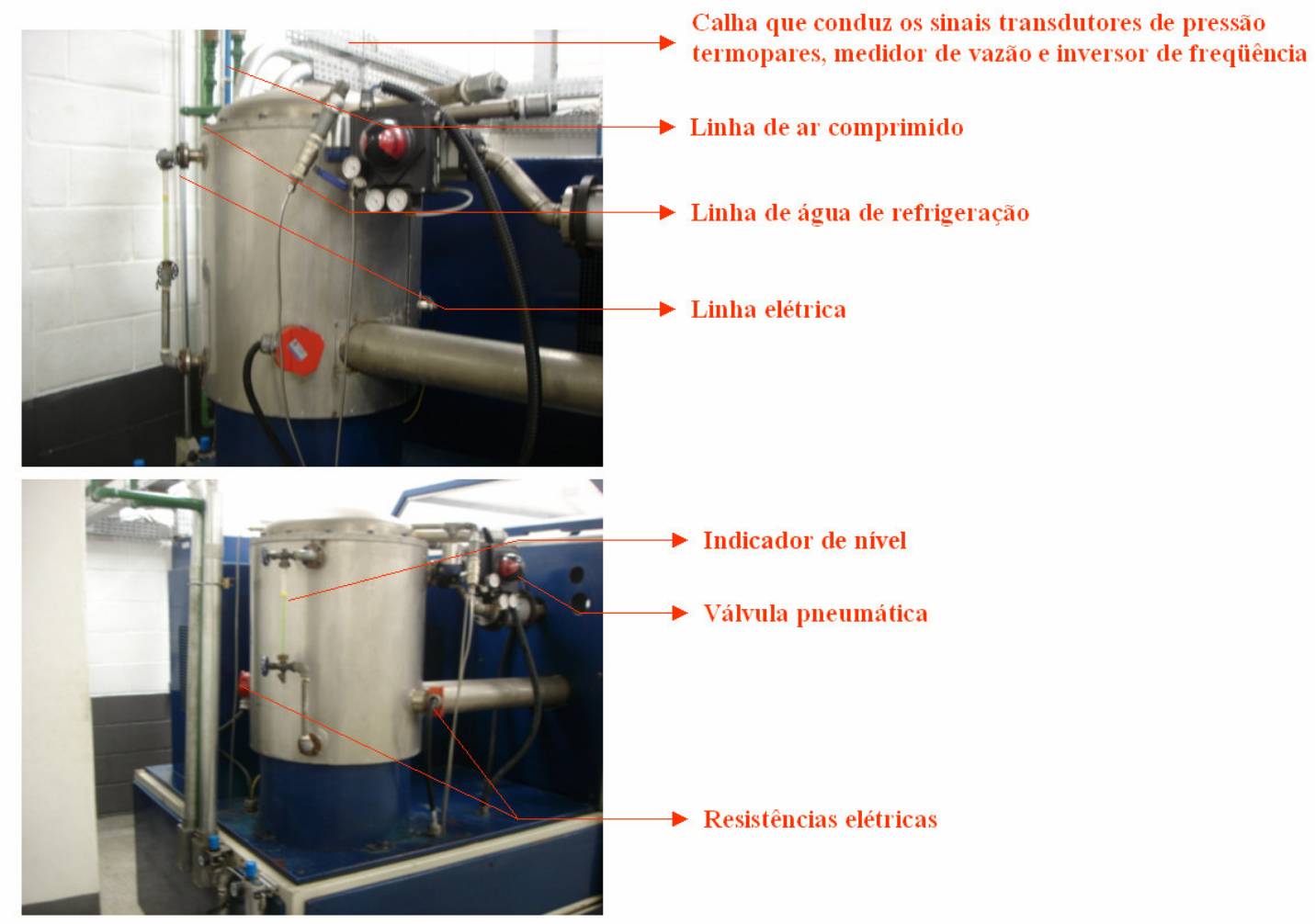

Figura 25 - Reservatório, Válvula Proporcional, Resistências, e Linhas de Ar Comprimido e Água de Refrigeração.

d) Um medidor de vazão eletromagnético de 3" e capacidade máxima de $9.46 \mathrm{l} / \mathrm{s}$, está montado a uma distancia de $2 \mathrm{~m}$ da saída da bomba (aproximadamente 25 vezes o diâmetro da tubulação). Ele possui um 
revestimento interno de PTFE, e suporta temperaturas de até $120^{\circ} \mathrm{C}$. A Figura 26 detalha o medidor de vazão.

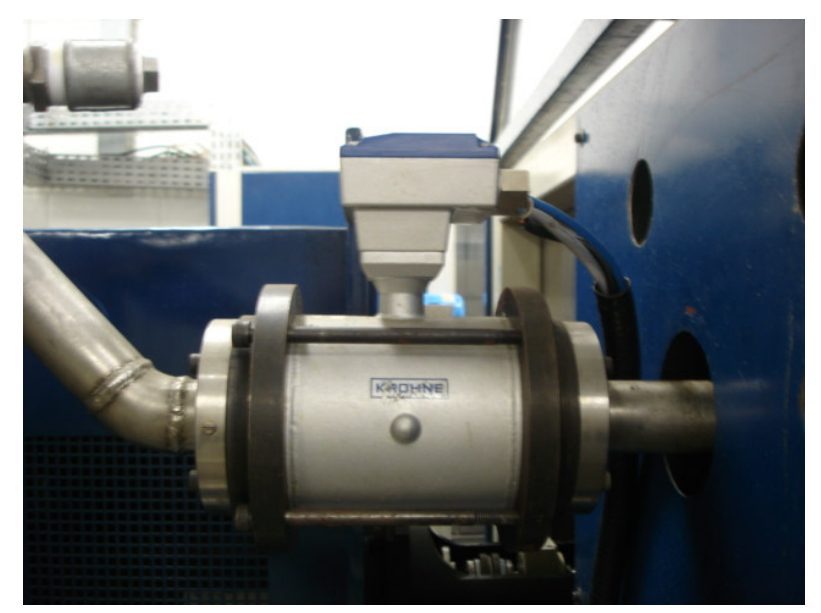

Figura 26 - Medidor de Vazão Eletromagnético

e) Três termopares tipo K, com a perna positiva feita em níquel-cromo, e a negativa em níquel-alumínio, tem a faixa de trabalho de -200 a $1200^{\circ} \mathrm{C}$ e podem operar tanto em meios oxidantes como inertes. Três transdutores de pressão que trabalham de -1 a 20 bar, com um sinal de saída de 4 a 20mA respectivamente. A Figura 30 mostra o posicionamento dos termopares e dos transdutores de pressão. A imagem do lado esquerdo mostra a frente enquanto a do lado mostra o fundo do equipamento

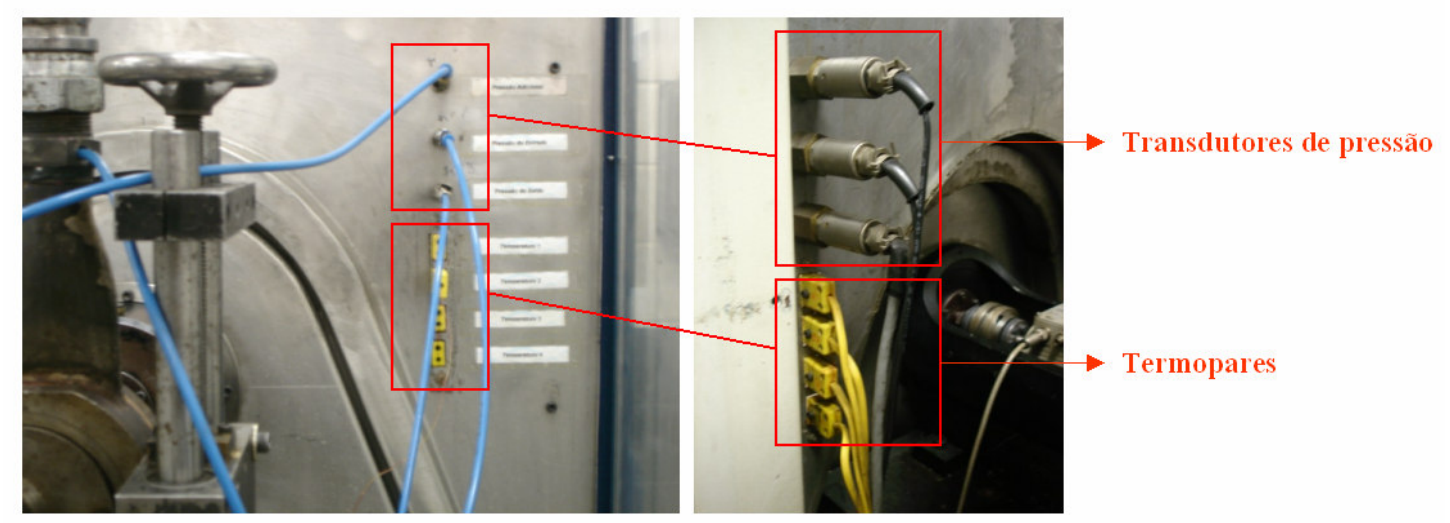

Figura 27 - Termopares e Transdutores de Pressão 
f) Um dispositivo de alumínio usinado com as mesmas dimensões do bloco do motor, de forma a refletir a interface da bomba com o motor. A Figura 28 mostra tal dispositivo.
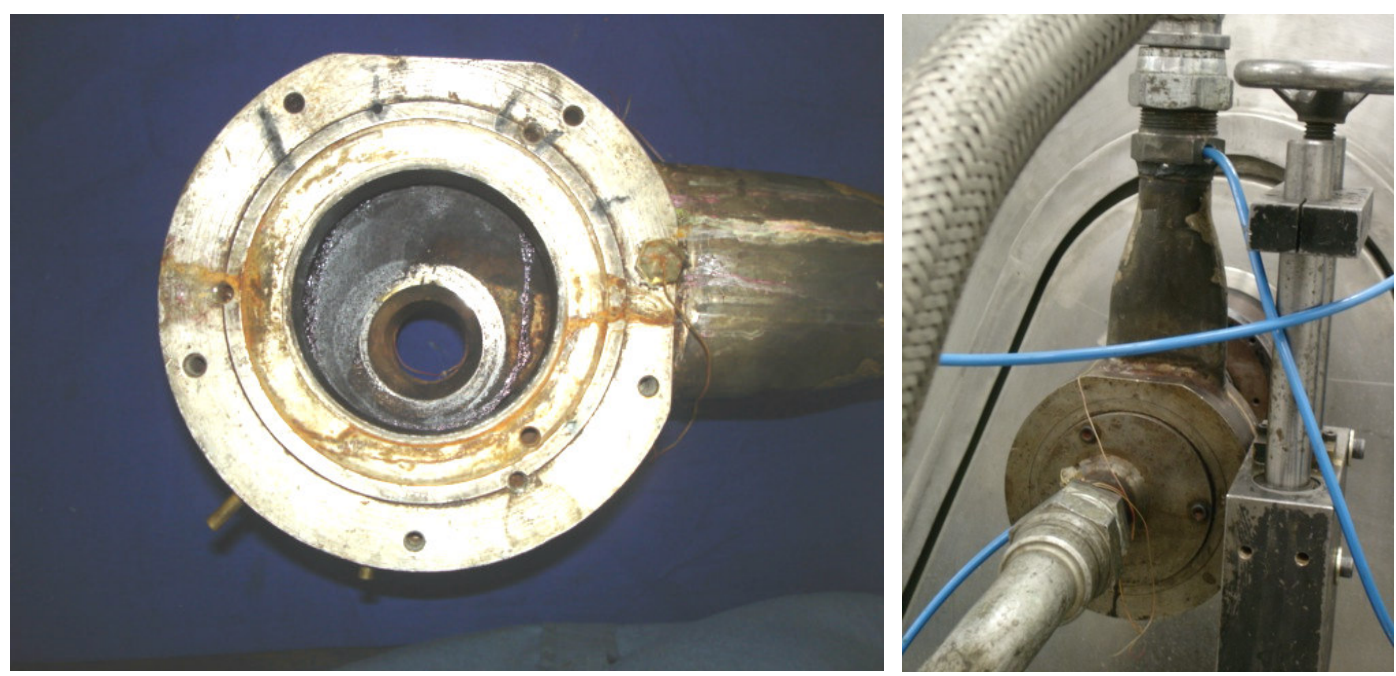

Figura 28 - Dispositivo de Interface

g) Um motor elétrico de 7,5 kW de potência, e rotação máxima de $8000 \mathrm{rpm}$ quando alimentado a $230 \mathrm{~V}, 60 \mathrm{~Hz}$ e 19,9A, utilizado para acionar a bomba. Sua rotação é controlada através de um inversor de freqüência, que trabalha com sinais PWM convertendo corrente alternada em corrente continua. Então a corrente continua é modulada e transformada em três fases de corrente alternada para o motor. Dessa forma o inversor converte um sinal fixo de freqüência em uma saída variável de freqüência que causa a variação na rotação operacional do motor. A Figura 29 apresenta o motor no lado esquerdo e o inversor de freqüência à direita. 

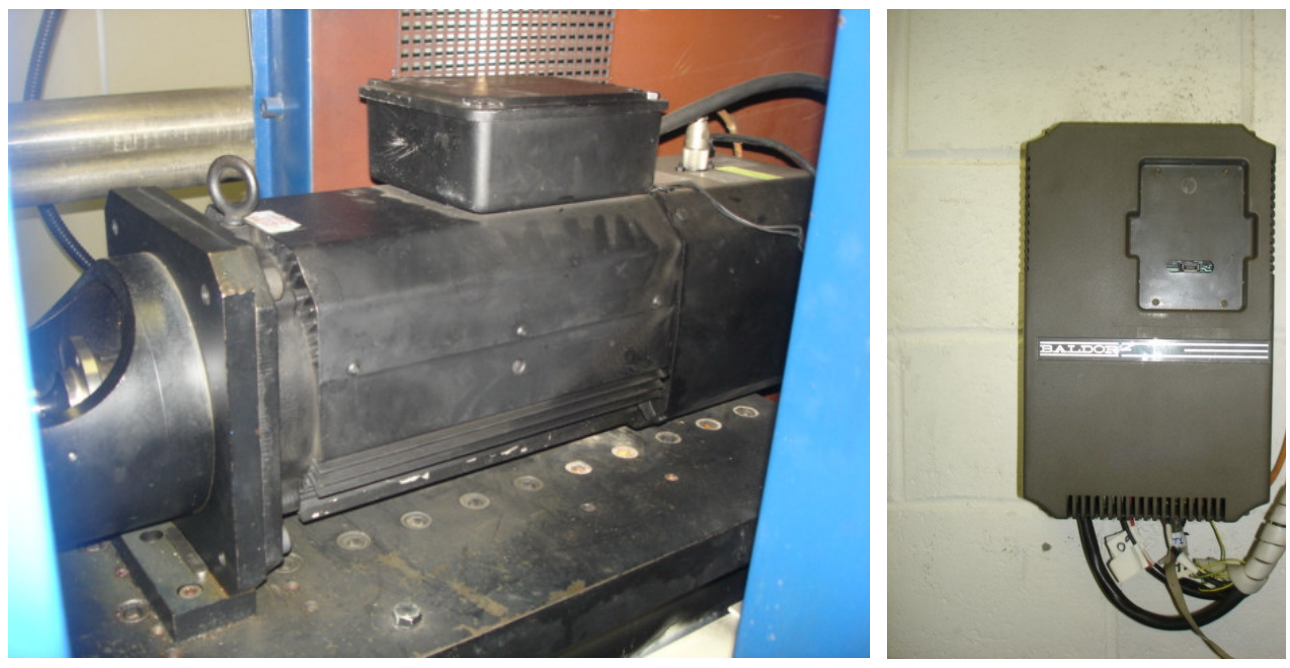

Figura 29 - Motor e Inversor de Freqüência

h) Uma célula de torque com limite de $20 \mathrm{~N} . \mathrm{m}$, com sinal de saída de $5 \mathrm{kHz}$ a $15 \mathrm{khz}$, onde $5 \mathrm{kHz}$ corresponde a um sinal de torque negativo, $10 \mathrm{kHz}$ corresponde a zero e $15 \mathrm{kHz}$ a um sinal de torque positivo. A célula é montada entre o motor e a bomba, e é utilizada para medir o torque de acionamento e a potência consumida. A Figura 30 ilustra o componente.

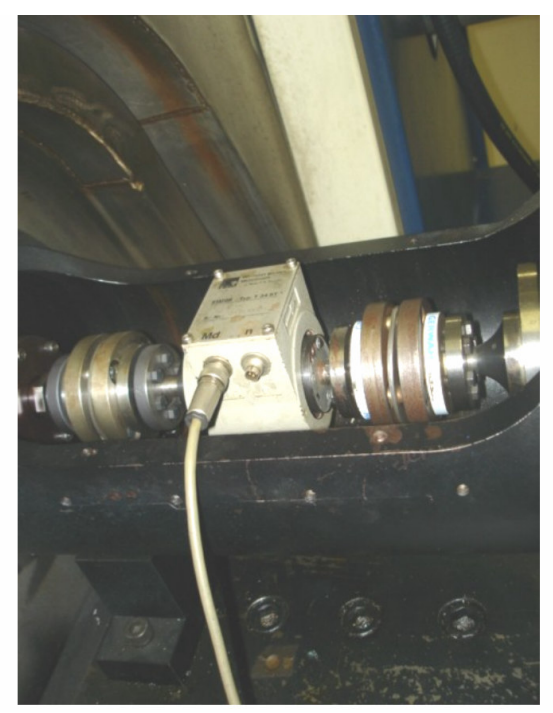

Figura 30 - Célula de Torque 
i) Um painel, mostrado na Figura 31, com controladores digitais que processam os sinais dos equipamentos descritos acima, e geram como saída, valores expressos em unidades de medidas físicas.
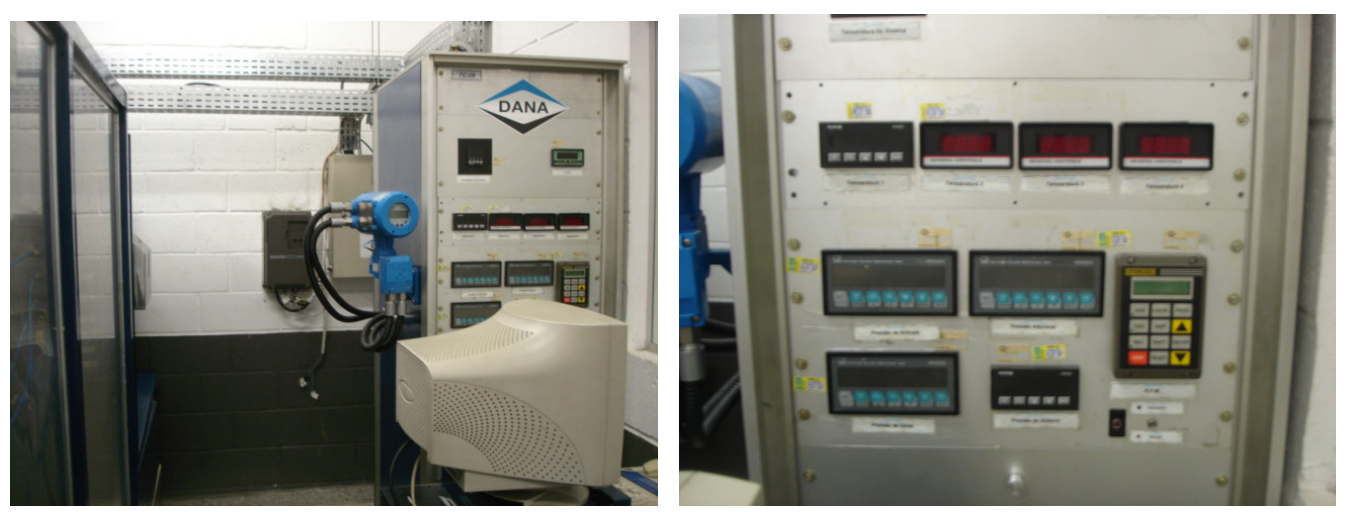

Figura 31- Controladores 


\subsection{Simulação em bancada da temperatura de cavitação do sistema de arrefecimento}

\subsubsection{Condições de Teste}

Este estudo examina a temperatura de cavitação de uma bomba d'água para concentrações distintas da mistura etilenoglicol / água, simulando diferentes condições de pressurização do sistema. As associações entre as concentrações da mistura etilenoglicol / água, pressão do sistema e rotação da bomba, testadas neste experimento, são descritas na Tabela 1.

Tabela 1 - Associações Testadas

\begin{tabular}{|c|c|c|}
\hline $\begin{array}{l}\text { Concentração das mistura etilenoglicol / água } \\
{[\%]}\end{array}$ & $\begin{array}{l}\text { Pressão do } \\
\text { Sistema [kPa] }\end{array}$ & $\begin{array}{l}\text { Rotação da } \\
\text { Bomba [rpm] }\end{array}$ \\
\hline $50 / 50$ & 50 & $\begin{array}{l}1000 \\
2000 \\
3000 \\
4000 \\
5000\end{array}$ \\
\hline $50 / 50$ & 15 & $\begin{array}{l}1000 \\
2000 \\
3000 \\
4000 \\
5000\end{array}$ \\
\hline $30 / 70$ & 50 & $\begin{array}{l}1000 \\
2000 \\
3000 \\
4000 \\
5000\end{array}$ \\
\hline $30 / 70$ & 15 & $\begin{array}{l}1000 \\
2000 \\
3000 \\
4000 \\
5000\end{array}$ \\
\hline $0 / 100$ & 50 & $\begin{array}{l}1000 \\
2000 \\
3000 \\
4000 \\
5000\end{array}$ \\
\hline $0 / 100$ & 15 & $\begin{array}{l}1000 \\
2000 \\
3000 \\
4000 \\
5000\end{array}$ \\
\hline
\end{tabular}


As condições de pressurização do sistema foram determinadas através do resultado do estudo de campo descrito no parágrafo 3.1, ou seja, a pressão de $15 \mathrm{kPa}$ representa a condição de operação, onde a válvula termostática do sistema de arrefecimento está prestes a abrir e a temperatura do fluido no by pass chega a algo em torno de $93^{\circ} \mathrm{C}$. Porém como o fluido ainda não circula pelo radiador, a pressão do sistema é baixa, propiciando condições favoráveis à ocorrência de cavitação (alta temperatura em condições de baixa pressão). A pressão de $50 \mathrm{kPa}$ representa a condição do sistema para a maior temperatura de trabalho, aproximadamente $104^{\circ} \mathrm{C}$, quando o veiculo saiu da estrada e voltou a trafegar em área urbana. Esse cenário pode ser observado, e melhor compreendido, através da análise da Figura 23

Todas as associações entre a concentração da mistura e a pressão do sistema foram testadas com a bomba trabalhando entre 1000 e $5000 \mathrm{rpm}$, com um passo de $1000 \mathrm{rpm}$. É importante lembrar que quanto maior for a rotação da bomba, menor é a pressão em sua entrada, e conseqüentemente, maior é a propensão à ocorrência de cavitação.

\subsubsection{Análise de incertezas}

O objetivo deste tópico é identificar e classificar os valores numéricos das incertezas associadas aos instrumentos utilizados no trabalho. A análise detalhada e os resultados obtidos são apresentados no Anexo A.

As incertezas são classificadas pela forma com a qual o valor numérico é determinado. Se dados estatísticos estão disponíveis, a incerteza é classificada como Tipo A, e como Tipo B quando não existe tal informação. Neste trabalho, todas as incertezas são classificadas como Tipo B, pois não existem dados estatísticos disponíveis e definidas por: 
onde $\pm \alpha$ define limites dentre os quais se espera encontrar 0 valor verdadeiro.

A incerteza expressa com um determinado nível de confiança é dado por k.u, onde k é o fator de abrangência. Valores numéricos de k são dados na tabela 2.

Tabela 2 - Fator de Abrangência

\begin{tabular}{|c|c|}
\hline Nível de Confiança (\%) & Fator de Abrangência - k \\
\hline 68,27 & 1,000 \\
\hline 90,00 & 1,645 \\
\hline 95,00 & 1,960 \\
\hline 95,45 & 2,000 \\
\hline 99,00 & 2,576 \\
\hline 99,73 & 3,000 \\
\hline
\end{tabular}

3.4.1 Incertezas Associadas ao Mapeamento dos Parâmetros de Funcionamento do Sistema de Arrefecimento.

Termopar tipo $\mathrm{K}$ :

$u_{T}=0,580 \times 0,400 \%=0,232 \%$

Então para um nível de confiança de $95 \%$, a incerteza será

$\pm 1,96 \times 0,232 \%=0,455 \%$

Transdutores de pressão:

$u_{p}=0,580 \times 0,300 \%=0,174 \%$

Então para um nível de confiança de $95 \%$, a incerteza será $\pm 1,96 \times 0,174 \%=0,341 \%$ 


\subsubsection{Incertezas Associadas a Simulação em Bancada}

Termopar tipo K:

$u_{T}=0,580 \times 0,400 \%=0,232 \%$

Então para um nível de confiança de $95 \%$, a incerteza será

$\pm 1,96 \times 0,232 \%=0,455 \%$

Transdutores de pressão:

$u_{p}=0,580 \times 0,300 \%=0,174 \%$

Então para um nível de confiança de $95 \%$, a incerteza será

$\pm 1,96 \times 0,174 \%=0,341 \%$

Incerteza Composta Associada à Medição do Diferencial de Pressão:

$D p=P_{2}-P_{1}$

Um coeficiente de sensibilidade deve ser determinado para cada uma das duas variáveis $P_{1}$ e $P_{2}$, porque os componentes de incerteza são associados no procedimento de analise de DP. A definição matemática de um componente de sensibilidade pode ser dado, considerando que uma determinada saída y, que é função de $n$ entradas:

$Y=y(x 1, x 2, x 3, x 4, \ldots, x n)$

O coeficiente de sensibilidade é dado por

$S_{x i}=\left(\frac{\partial y}{\partial x_{i}}\right)\left(\frac{x_{i}}{y}\right)$

O coeficiente de sensibilidade pode ser interpretado como a mudança percentual em DP que resulta da mudança de $1 \%$ na variável. 
Então o coeficiente de sensibilidade para $\mathrm{P}_{1}$ é:

$S_{P_{1}}=\left(\frac{\partial D P}{\partial P_{1}}\right)\left(\frac{P_{1}}{D P}\right)=-\frac{P_{1}}{P_{2}-P_{1}}$

e para P2 é:

$S_{P_{2}}=\left(\frac{\partial D P}{\partial P_{2}}\right)\left(\frac{P_{2}}{D P}\right)=\frac{P_{2}}{P_{2}-P_{1}}$

a incerteza combinada é dada pela equação:

$\left(\frac{u(y)}{y}\right)^{2}=\sum_{i=1}^{n} S_{x_{i}}^{2} u^{2}\left(x_{i}\right)$

\subsection{Resultados}

As figuras a seguir mostram curvas que expressam a diferença de pressão entre a saída e a entrada da bomba $(\Delta P)$ em função da temperatura do fluido. Para a realização do experimento, a válvula proporcional detalhada no item "c)" do parágrafo 3.2 é ajustada em $50 \%$ de sua abertura total e assim mantida em todas as condições de teste. Então, para cada rotação existe uma diferença de pressão $\Delta P$ que identifica a ocorrência de cavitação quando esta sofre uma queda de $5 \%$. Esta condição é representada nos gráficos como o ponto DP5\%. Algumas curvas trazem também um ponto chamado DP10\%, que representa uma queda de $10 \%$ no diferencial de pressão e indica uma condição de cavitação mais severa.

É importante salientar que o fenômeno de cavitação é função apenas da rotação de trabalho da bomba, da temperatura do fluido, e da concentração de etilenoglicol na água, que impacta diretamente na pressão de vapor da 
mistura. Ou seja, não importa se a bomba trabalha com um diferencial de pressão de 100 ou $10 \mathrm{kPa}$, a suscetibilidade à cavitação dependerá apenas dos fatores citados acima.

A máxima temperatura experimentada foi $110^{\circ} \mathrm{C}$, por limitação da bancada, e por ter sido evidenciado no parágrafo 3.1 que as temperaturas em um sistema de arrefecimento convencional não superam os $105^{\circ} \mathrm{C}$.

A Figura 32 expressa os resultados da simulação do sistema de arrefecimento em bancada trabalhando com uma mistura $50 \%$ etilenoglicol e $50 \%$ água, com pressão de sistema de $50 \mathrm{kPa}$. Pode-se ver que mesmo aumentando a temperatura do fluido para $110^{\circ} \mathrm{C}$, a cavitação não ocorre para rotações de até $3000 \mathrm{rpm}$. Porém, evidencia-se a ocorrência do fenômeno para as rotações de 4000 e 5000 rpm, sendo que as temperaturas de cavitação foram respectivamente 107,6 e 107,0 C.

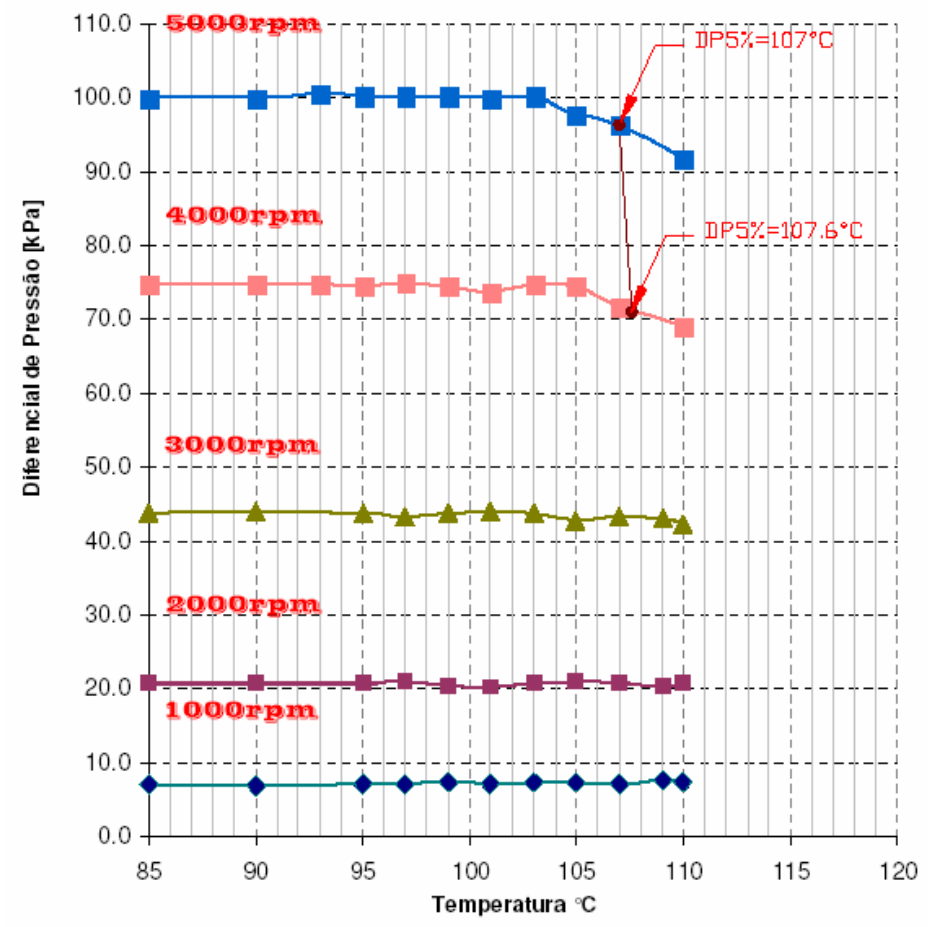

Figura 32 - Mistura de $\mathbf{5 0 \%}$ Etilenoglicol e $\mathbf{5 0 \%}$ água, com pressão de sistema de $50 \mathrm{kPa}$ 
É esperado que a temperatura de cavitação para 4000 rpm seja maior que para $5000 \mathrm{rpm}$. Porém, para $5000 \mathrm{rpm}$ com a mistura de $50 \%$ etilenoglicol e $50 \%$ água e $50 \mathrm{kPa}$ de pressão de sistema a incerteza combinada no ponto DP5\% é de $2,6 \%$, o que indica que o diferencial de pressão medido (96,3 $\mathrm{kPa}$ ) pode variar entre $97,7 \mathrm{kPa}$ e $95,1 \mathrm{kPa}$, o que reflete em uma variação na temperatura de cavitação de $105{ }^{\circ} \mathrm{C}$ a $108{ }^{\circ} \mathrm{C}$ aproximadamente. Seguindo o mesmo raciocínio para a rotação de 4000 rpm, a temperatura de cavitação varia entre 101 e $109^{\circ} \mathrm{C}$, onde para este ponto a incerteza combinada é $3,1 \%$. A Figura 33 mostra a sobreposição das faixas onde a temperatura de cavitação pode se encontrar, para as rotações de $4000 \mathrm{e}$ $5000 \mathrm{rpm}$. É importante salientar que a "espessura da faixa" depende do valor da incerteza como também do gradiente de DP em função da temperatura.

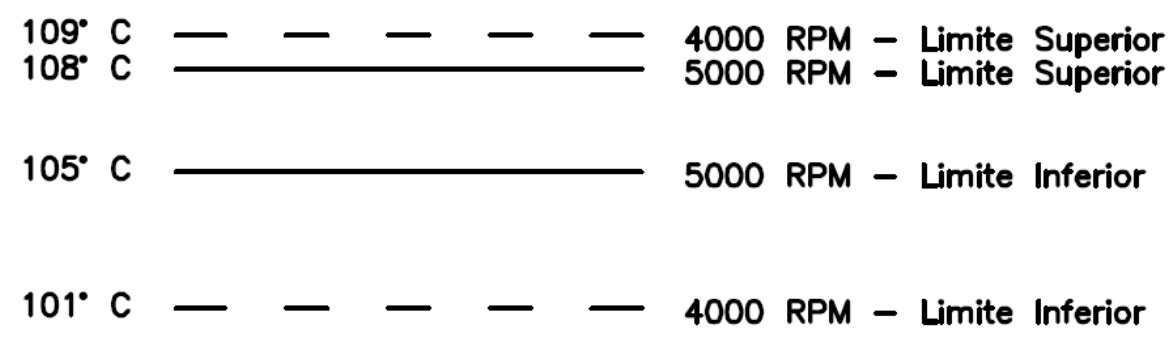

Figura 33 - Temperatura de cavitação para a mistura 50\% etilenoglicol e $50 \%$ água, a $50 \mathrm{kPa}$ de pressão de sistema, ponderando as incertezas

A Figura 34 traz o resultado da simulação para a mesma concentração de $50 \%$ etilenoglicol e 50\% água, porém com a pressão de sistema de $15 \mathrm{kPa}$. Pode-se verificar que para esta condição a temperatura de cavitação cai consideravelmente para as rotações de 5000 e $4000 \mathrm{rpm}$, e que para 3000 rpm temos agora a ocorrência de cavitação, fato não verificado quando trabalhando com a pressão de sistema de $50 \mathrm{kPa}$. Pode-se ainda verificar que para esta condição chega-se facilmente ao ponto DP10\%, algo não atingido anteriormente. A diferença entre a temperatura de cavitação (DP5\%) para as diferentes faixas de rotação também aumentou, ficando $2^{\circ} \mathrm{C}$ 
entre 5000 e 4000rpm e $4^{\circ} \mathrm{C}$ para 4000 e 3000 rpm. Não foi evidenciada a ocorrência de cavitação para as rotações de 2000 e 1000 rpm.

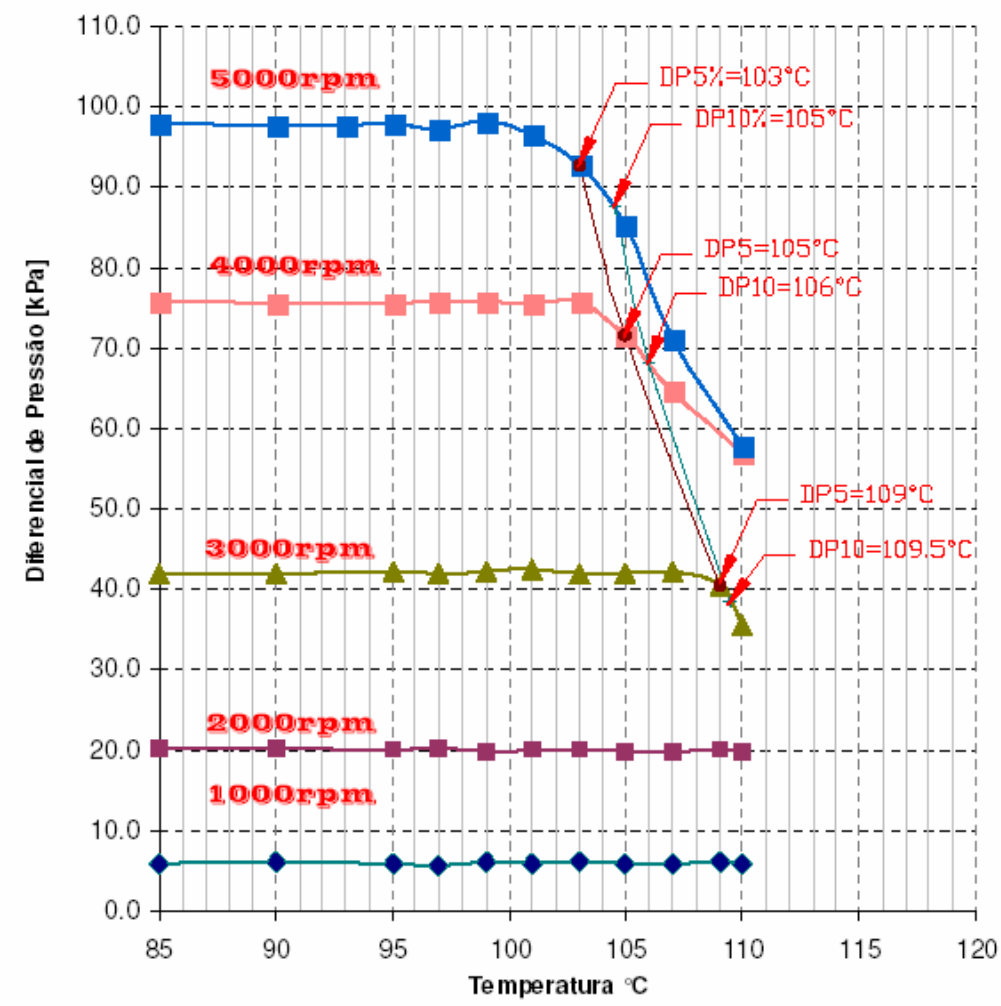

Figura 34 - Mistura de $50 \%$ Etilenoglicol e $\mathbf{5 0 \%}$ água, com pressão de sistema de $15 \mathrm{kPa}$

Para a mistura $50 \%$ etilenoglicol e $50 \%$ água, a $15 \mathrm{kPa}$ de pressão de sistema a $5000 \mathrm{rpm}$, o ponto DP5\% tem incerteza combinada de 2,0\%, o que significa que a temperatura de cavitação para esta condição pode estar entre $102^{\circ} \mathrm{C}$ e $104^{\circ} \mathrm{C}$. Na rotação de $4000 \mathrm{rpm}$, o ponto DP5\% tem incerteza combinada de $2,0 \%$, ou seja, temperatura de cavitação entre $105^{\circ} \mathrm{C}$ e $107^{\circ}$ C. Em $3000 \mathrm{rpm}$, o ponto DP5\% tem incerteza combinada de 2,5\%, refletindo em uma temperatura de cavitação entre $105^{\circ} \mathrm{C}$ e $109^{\circ}$. A Figura 35 mostra a sobreposição das faixas de incerteza. 


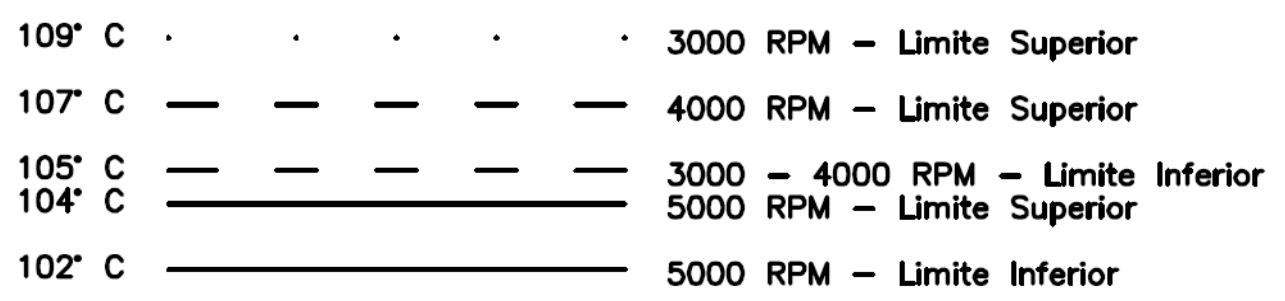

Figura 35 - Temperatura de cavitação para a mistura $50 \%$ etilenoglicol e $50 \%$ água, a $15 \mathrm{kPa}$ de pressão de sistema, ponderando as incertezas

Os resultados da simulação para a mistura de $30 \%$ etilenoglicol e $70 \%$ água com a pressão de sistema de $50 \mathrm{kPa}$ são mostrados na Figura 36. Esta configuração mostrou-se menos suscetível a ocorrência de cavitação que a concentração de 50 \% etilenoglicol e 50 \% água com pressão de sistema de $15 \mathrm{kPa}$, porém mais propensa à ocorrência do fenômeno que a concentração de $50 \%$ etilenoglicol e $50 \%$ água com pressão de sistema de $50 \mathrm{kPa}$. A cavitação ocorreu apenas para as rotações de 5000 e $4000 \mathrm{rpm}$, alcançando o ponto DP10\% em ambos os casos. A diferença para a temperatura de cavitação entre as duas rotações ficou em $2^{\circ} \mathrm{C}$. 


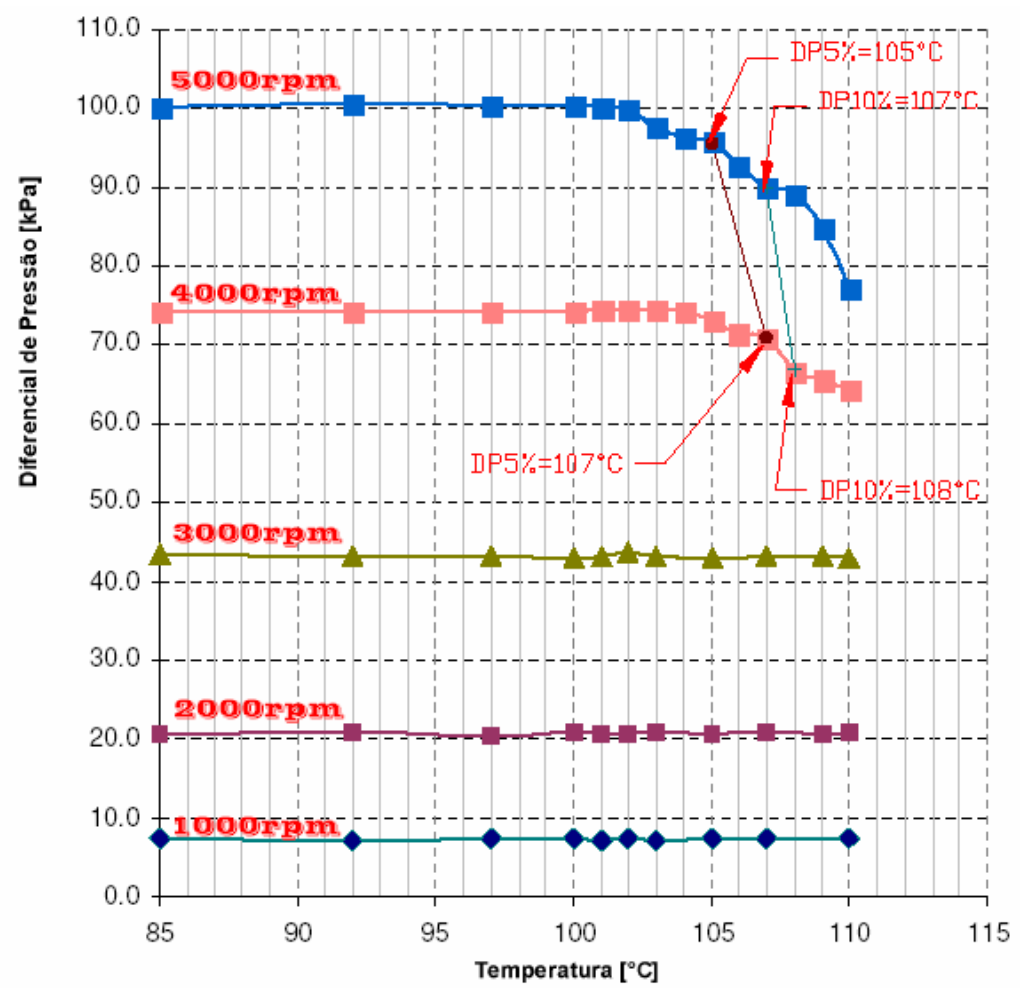

Figura 36 - Mistura de $30 \%$ Etilenoglicol e $70 \%$ água, com pressão de sistema de $50 \mathrm{kPa}$

Com a mistura $30 \%$ etilenoglicol e $70 \%$ água, a $50 \mathrm{kPa}$ de pressão de sistema a $5000 \mathrm{rpm}$, o ponto DP5\% tem incerteza combinada de $2,6 \%$, o que significa que a temperatura de cavitação para esta condição pode estar entre $103^{\circ} \mathrm{C}$ e $106^{\circ} \mathrm{C}$. Na rotação de $4000 \mathrm{rpm}$, o ponto DP5\% tem incerteza combinada de $3,0 \%$, ou seja, temperatura de cavitação entre $105^{\circ} \mathrm{C}$ e $108^{\circ}$ C. A Figura 37 mostra a sobreposição das faixas de incerteza.

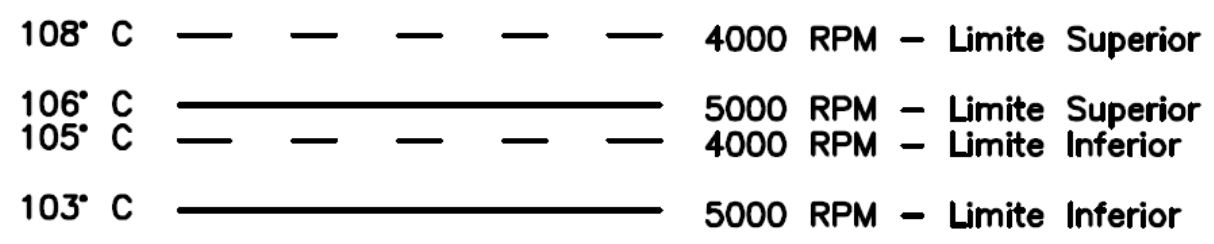

Figura 37 - Temperatura de cavitação para a mistura $30 \%$ etilenoglicol e $70 \%$ água, a $50 \mathrm{kPa}$ de pressão de sistema, ponderando as incertezas. 
A Figura 38 apresenta os resultados da simulação para a mistura de $30 \%$ etilenoglicol e $70 \%$ água com a pressão de sistema de $15 \mathrm{kPa}$. O ponto DP10\% foi atingido para as rotações de 5000, 4000 e 3000 rpm, sendo que a temperatura de cavitação, representada pelo ponto DP5\%, se deu respectivamente a 98,102 e $108^{\circ} \mathrm{C}$, ou seja, uma diferença de $4^{\circ} \mathrm{C}$ entre 5000 e $4000 \mathrm{rpm}$ e $6^{\circ} \mathrm{C}$ entre 4000 e $3000 \mathrm{rpm}$. Não houve cavitação para as rotações de 2000 e $1000 \mathrm{rpm}$.

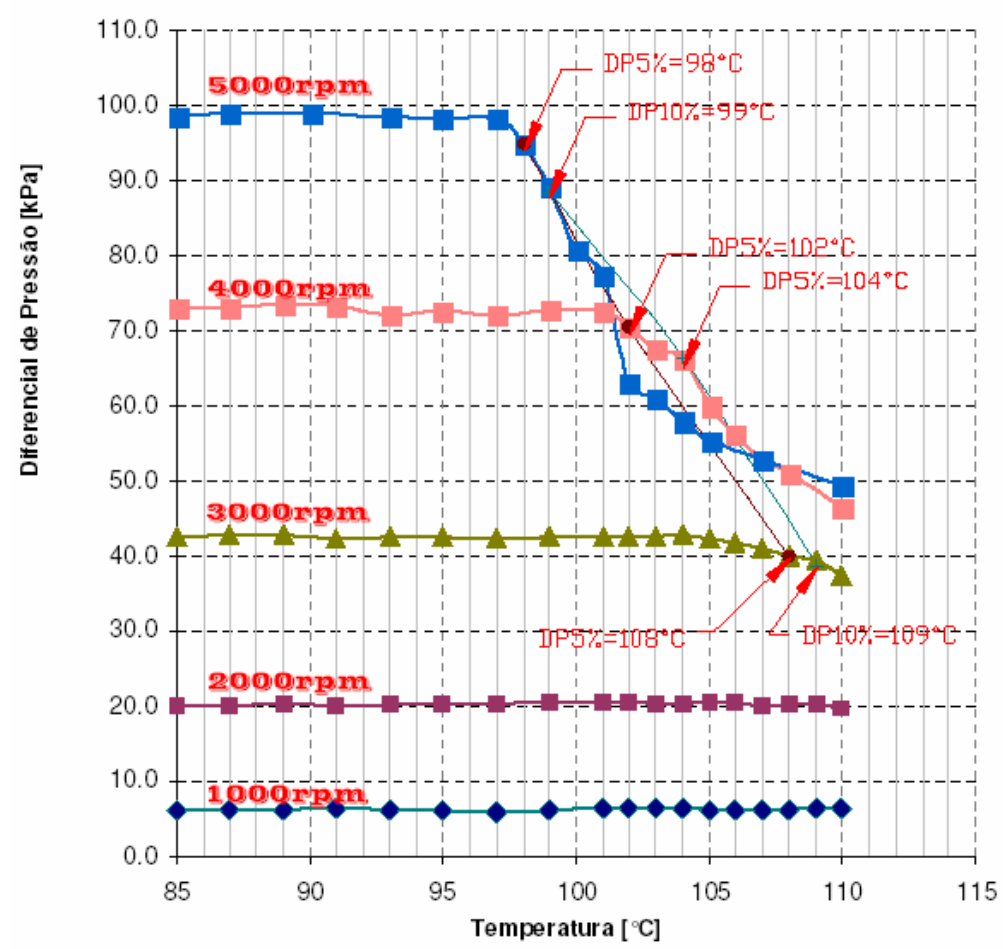

Figura 38- Mistura de 30\% Etilenoglicol e 70\% água, com pressão de sistema de $15 \mathrm{kPa}$

Na mistura 30\% etilenoglicol e $70 \%$ água, a $15 \mathrm{kPa}$ de pressão de sistema a $5000 \mathrm{rpm}$, o ponto DP5\% tem incerteza combinada de 1,9\%, o que significa que a temperatura de cavitação para esta condição pode estar entre $97^{\circ} \mathrm{C} \mathrm{e}$ $98^{\circ} \mathrm{C}$. Na rotação de $4000 \mathrm{rpm}$, o ponto DP5\% tem incerteza combinada de $2,1 \%$, ou seja, temperatura de cavitação entre $97^{\circ} \mathrm{C}$ e $102^{\circ} \mathrm{C}$. Em 3000 rpm, o ponto DP5\% tem incerteza combinada de $2,3 \%$, refletindo em uma 
temperatura de cavitação entre $107^{\circ} \mathrm{C}$ e $109^{\circ}$. A Figura 39 mostra a sobreposição das faixas de incerteza.

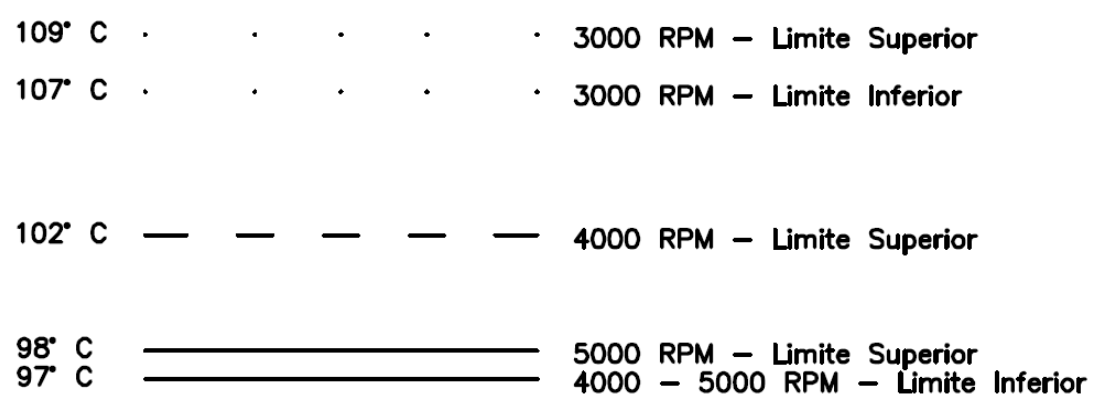

Figura 39 - Temperatura de cavitação para a mistura $30 \%$ etilenoglicol e $70 \%$ água, a $15 \mathrm{kPa}$ de pressão de sistema, ponderando as incertezas.

A Figura 40 traz os resultados dos testes feitos com $100 \%$ de água e pressão de sistema de $50 \mathrm{kPa}$. Cavitação ocorreu apenas paras as rotações de 5000, 4000 e 3000 rpm, e a temperatura de cavitação foi respectivamente 98, 104 e $107^{\circ} \mathrm{C}$. As diferenças de temperatura foram de $6^{\circ} \mathrm{C}$ entre as rotações de 5000 e $4000 \mathrm{rpm}$, e $3^{\circ} \mathrm{C}$ entre as rotações de 4000 e 3000 rpm. 


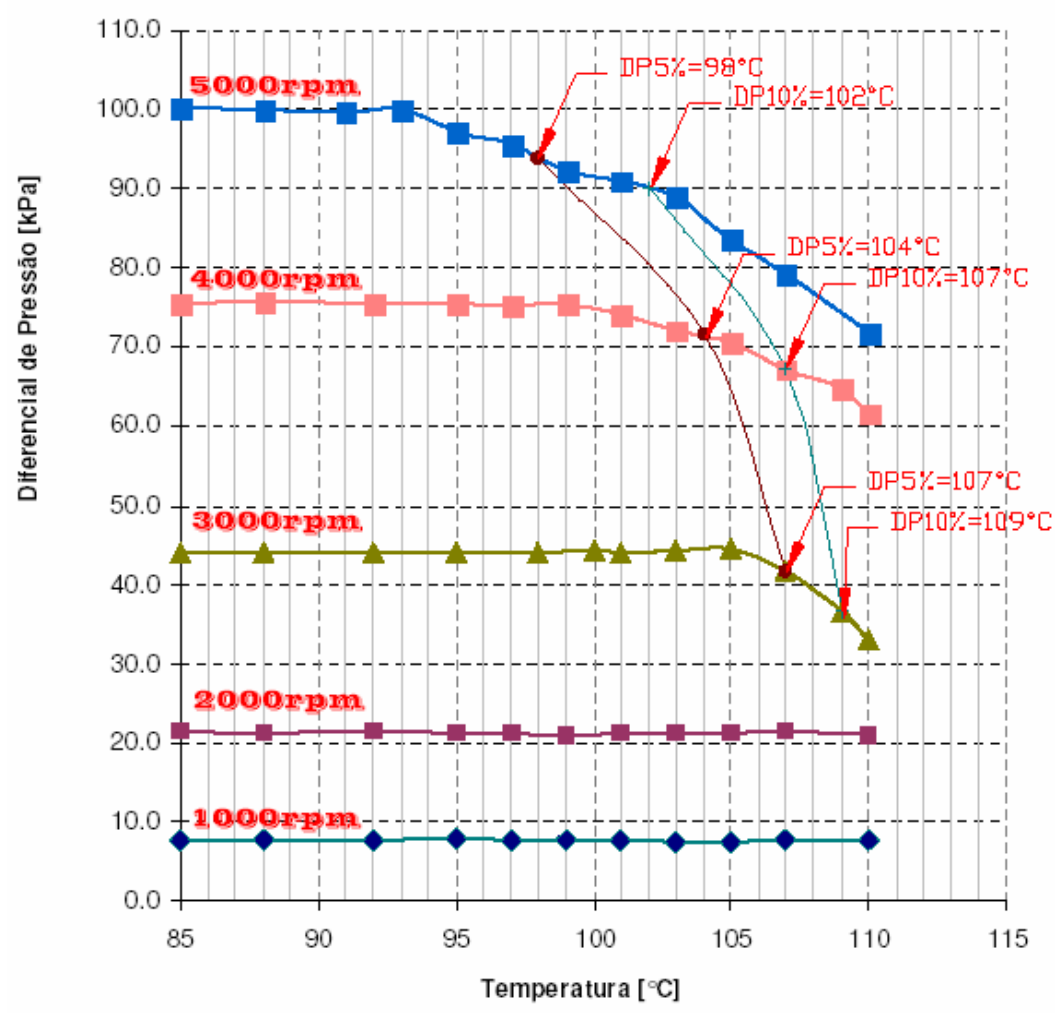

Figura 40-100\% água, com pressão de sistema de $50 \mathrm{kPa}$

Para $100 \%$ de água, com $50 \mathrm{kPa}$ de pressão de sistema a $5000 \mathrm{rpm}$, o ponto DP5\% tem incerteza combinada de 2,7\%, o que significa que a temperatura de cavitação para esta condição pode estar entre $97^{\circ} \mathrm{C}$ e $102^{\circ} \mathrm{C} . \mathrm{Na}$ rotação de 4000 rpm, o ponto DP5\% tem incerteza combinada de 2,9\%, ou seja, temperatura de cavitação entre $103^{\circ} \mathrm{C}$ e $107^{\circ} \mathrm{C}$. Em $3000 \mathrm{rpm}$, o ponto DP5\% tem incerteza combinada de 3,9\%, refletindo em uma temperatura de cavitação entre $105^{\circ} \mathrm{C}$ e $107^{\circ} \mathrm{C}$. A Figura 41 mostra a sobreposição das faixas de incerteza. 


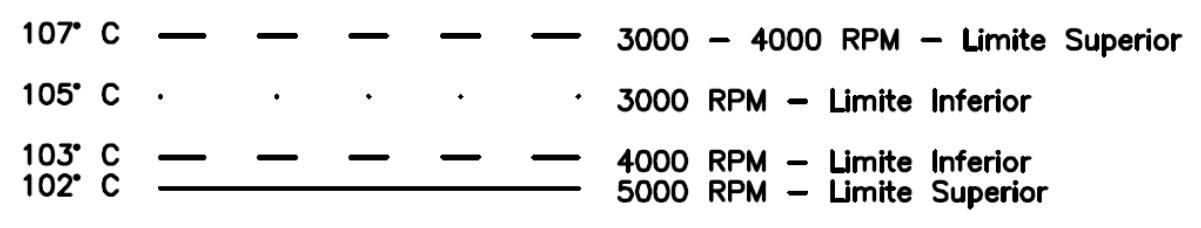

$97^{\circ} \mathrm{C} \longrightarrow 5000$ RPM - Limite Inferior

Figura 41 - Temperatura de cavitação para 100\% água, a $50 \mathrm{kPa}$ de pressão de sistema, ponderando as incertezas.

A Figura 42 representa os resultados para $100 \%$ de água e pressão de sistema de $15 \mathrm{kPa}$. Não houve cavitação para as rotações de 2000 e 1000 rpm. A temperatura de cavitação para as rotações de 5000, 4000 e 3000 rpm foram respectivamente 92,94 e $105^{\circ} \mathrm{C}$, e as diferenças de temperatura foram $2^{\circ} \mathrm{C}$ entre 5000 e 4000 , e $11^{\circ} \mathrm{C}$ entre 4000 e $3000 \mathrm{rpm}$.

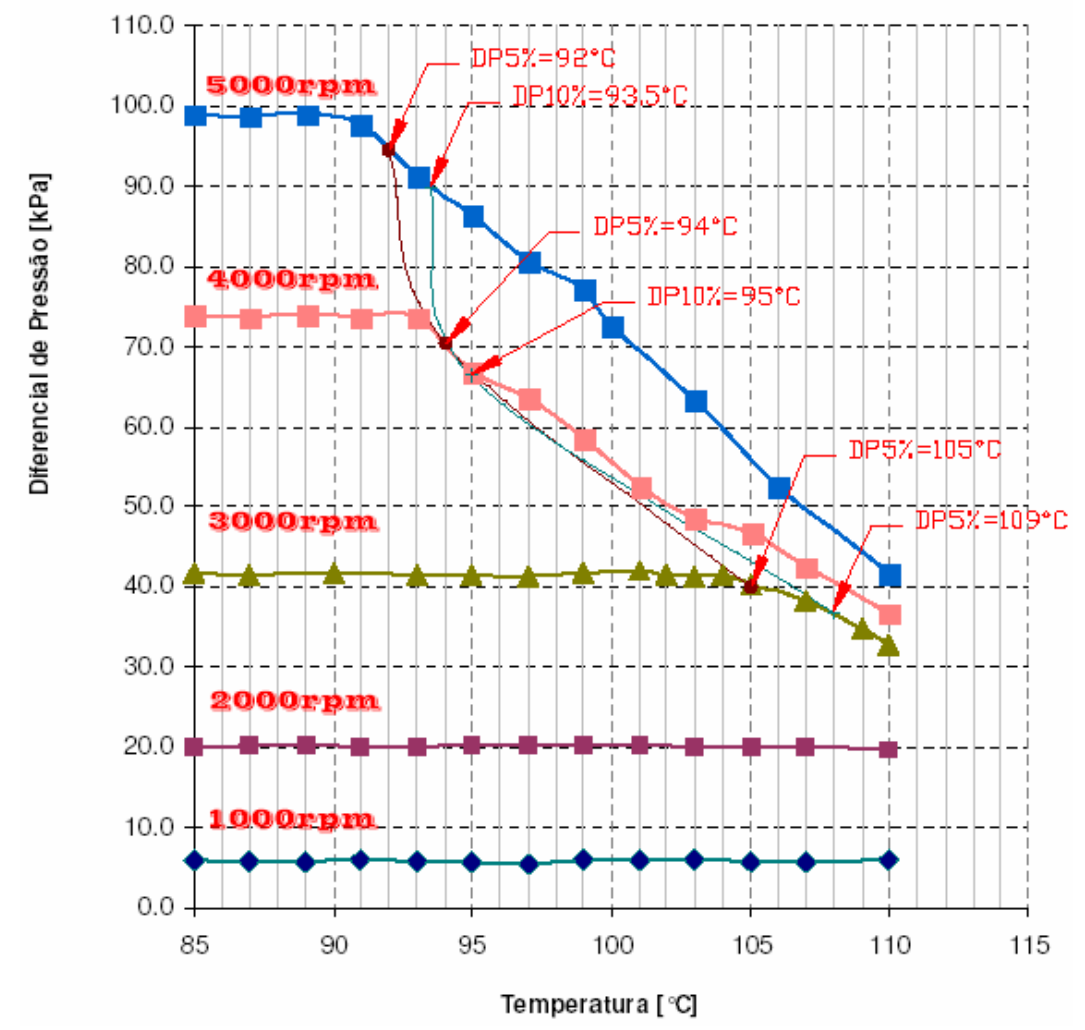

Figura $42-100 \%$ água, com pressão de sistema de $15 \mathrm{kPa}$ 
E com $100 \%$ de água, e $15 \mathrm{kPa}$ de pressão de sistema a $5000 \mathrm{rpm}$, o ponto DP5\% tem incerteza combinada de 1,9\%, o que significa que a temperatura de cavitação para esta condição pode estar entre $89^{\circ} \mathrm{C}$ e $92^{\circ} \mathrm{C}$. Na rotação de $4000 \mathrm{rpm}$, o ponto DP5\% tem incerteza combinada de 2,1\%, ou seja, temperatura de cavitação entre $94^{\circ} \mathrm{C}$ e $95^{\circ} \mathrm{C}$. Em $3000 \mathrm{rpm}$, o ponto DP5\% tem incerteza combinada de 2,3\%, refletindo em uma temperatura de cavitação entre $99^{\circ} \mathrm{C}$ e $106^{\circ} \mathrm{C}$. A Figura 43 mostra a sobreposição das faixas de incerteza.

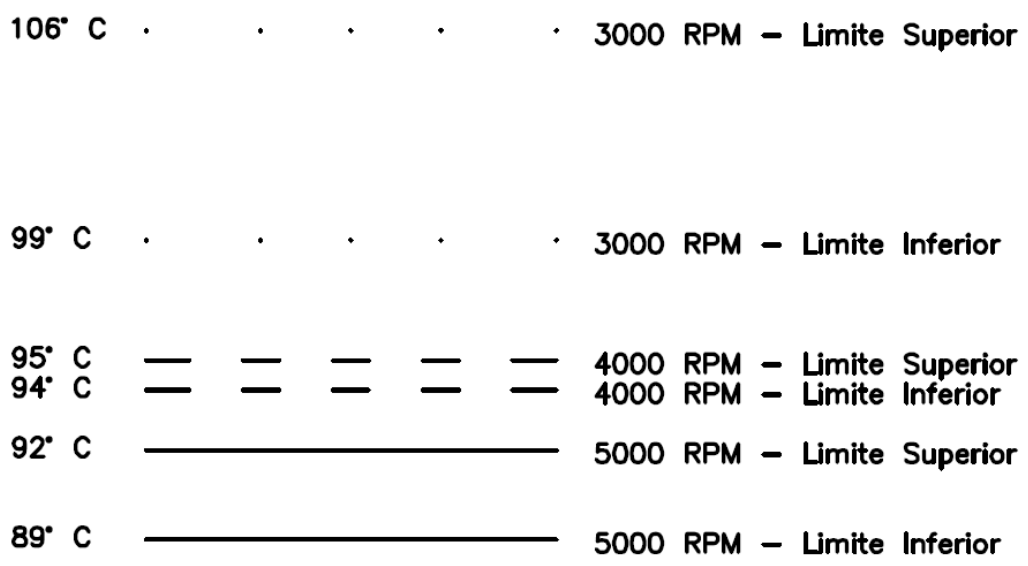

Figura 43 - Temperatura de cavitação para $100 \%$ água, a $50 \mathrm{kPa}$ de pressão de sistema, ponderando as incertezas.

A Figura 44 traz a comparação e identificação da temperatura de cavitação (DP5\%) para as concentrações de 50\% etilenoglicol e 50\% água, 30\% etilenoglicol e $70 \%$ água, e $100 \%$ água, com a bomba trabalhando a 5000 rpm e o sistema com pressão de $50 \mathrm{kPa}$. 


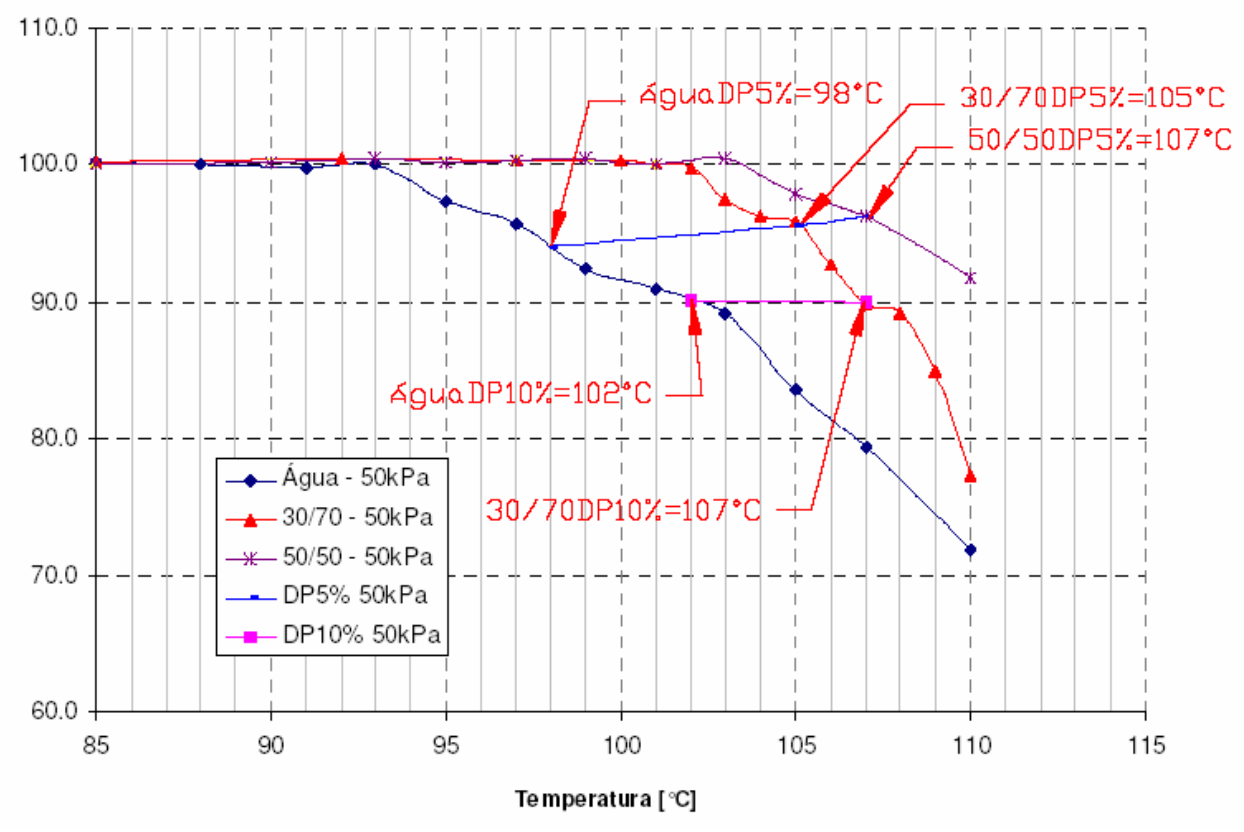

Figura 44 - Bomba a 5000 rpm e sistema com 50 kPa

A Figura 45 traz a comparação e identificação da temperatura de cavitação para as concentrações de 50\% etilenoglicol e 50\% água, 30\% etilenoglicol e $70 \%$ água, e 100\% água, com a bomba trabalhando a 5000 rpm e o sistema com pressão de $15 \mathrm{kPa}$.

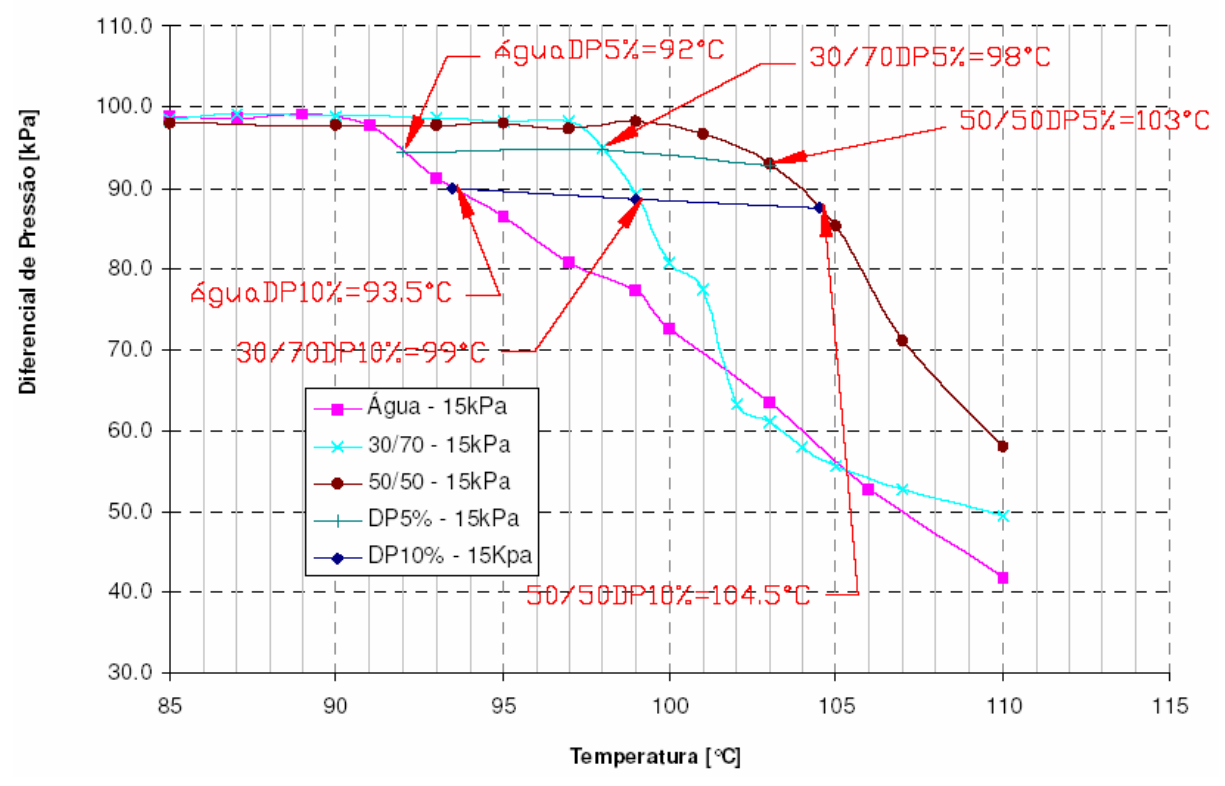

Figura 45 - Bomba a 5000 rpm e sistema com 15 kPa 
A Figura 49 traz a comparação e identificação da temperatura de cavitação para as concentrações de 50\% etilenoglicol e 50\% água, 30\% etilenoglicol e $70 \%$ água, e $100 \%$ água, com a bomba trabalhando a 4000 rpm e o sistema com pressão de $50 \mathrm{kPa}$.

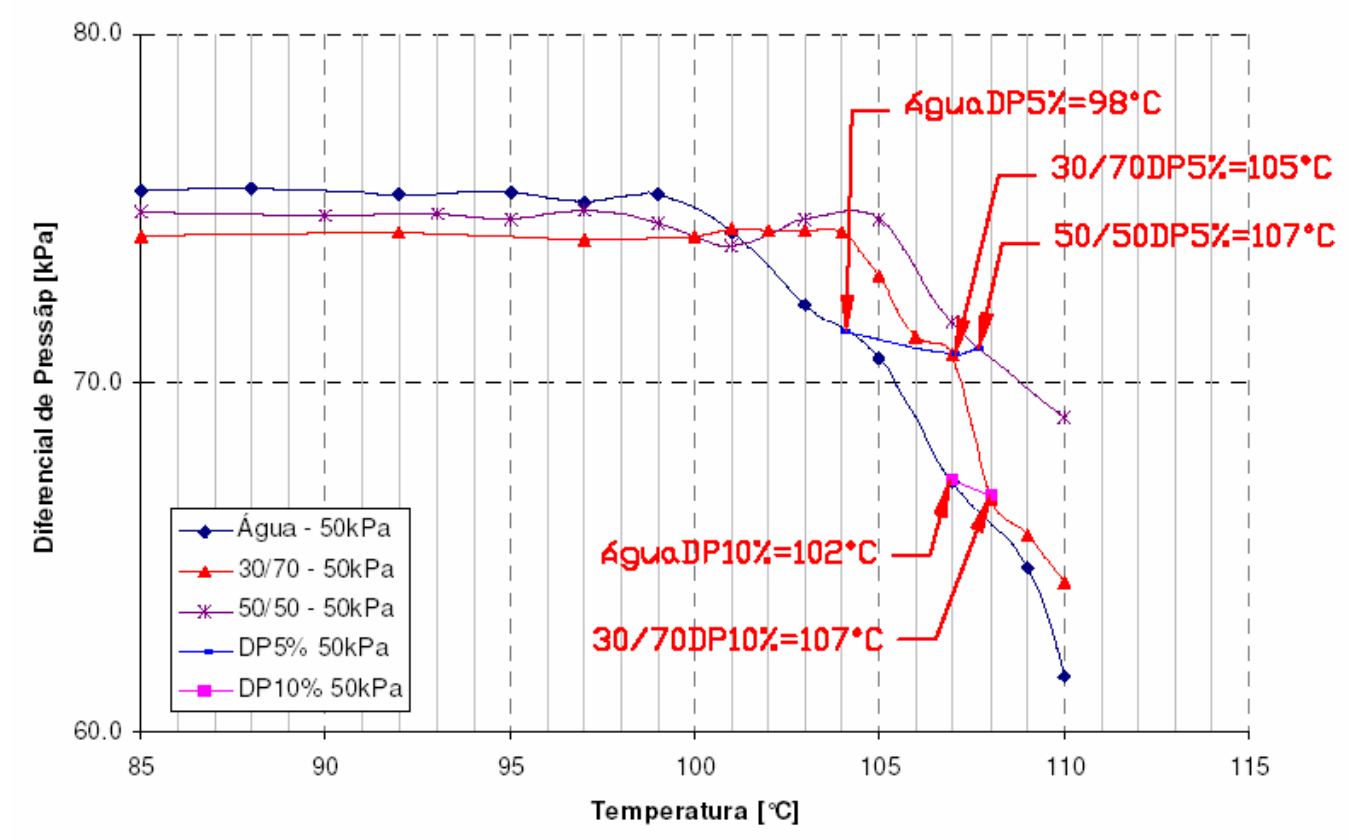

Figura 46 - Bomba a $4000 \mathrm{rpm}$ e sistema com $50 \mathrm{kPa}$

A Figura 47 traz a comparação e identificação da temperatura de cavitação para as concentrações de 50\% etilenoglicol e 50\% água, 30\% etilenoglicol e $70 \%$ água, e $100 \%$ água, com a bomba trabalhando a 4000 rpm e o sistema com pressão de $15 \mathrm{kPa}$. 


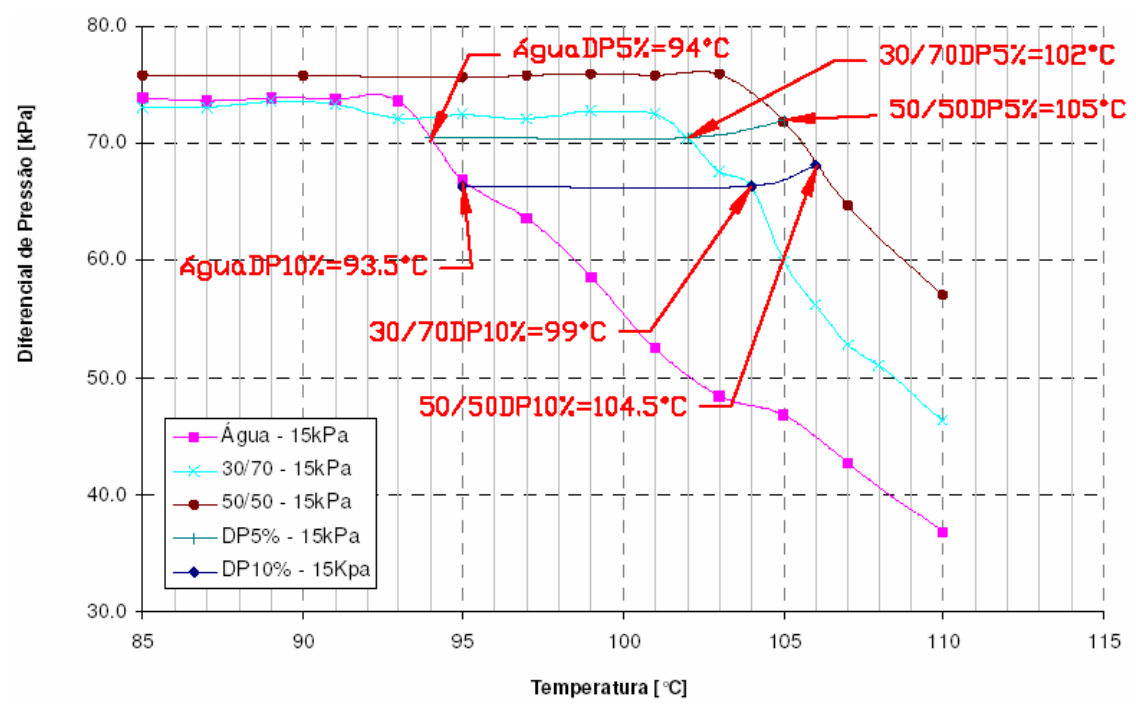

Figura 47 - Bomba a $4000 \mathrm{rpm}$ e sistema com $15 \mathrm{kPa}$

A Figura 48 traz a comparação e identificação da temperatura de cavitação para as concentrações de 50\% etilenoglicol e 50\% água, 30\% etilenoglicol e $70 \%$ água, e $100 \%$ água, com a bomba trabalhando a 3000 rpm e o sistema com pressão de $50 \mathrm{kPa}$.

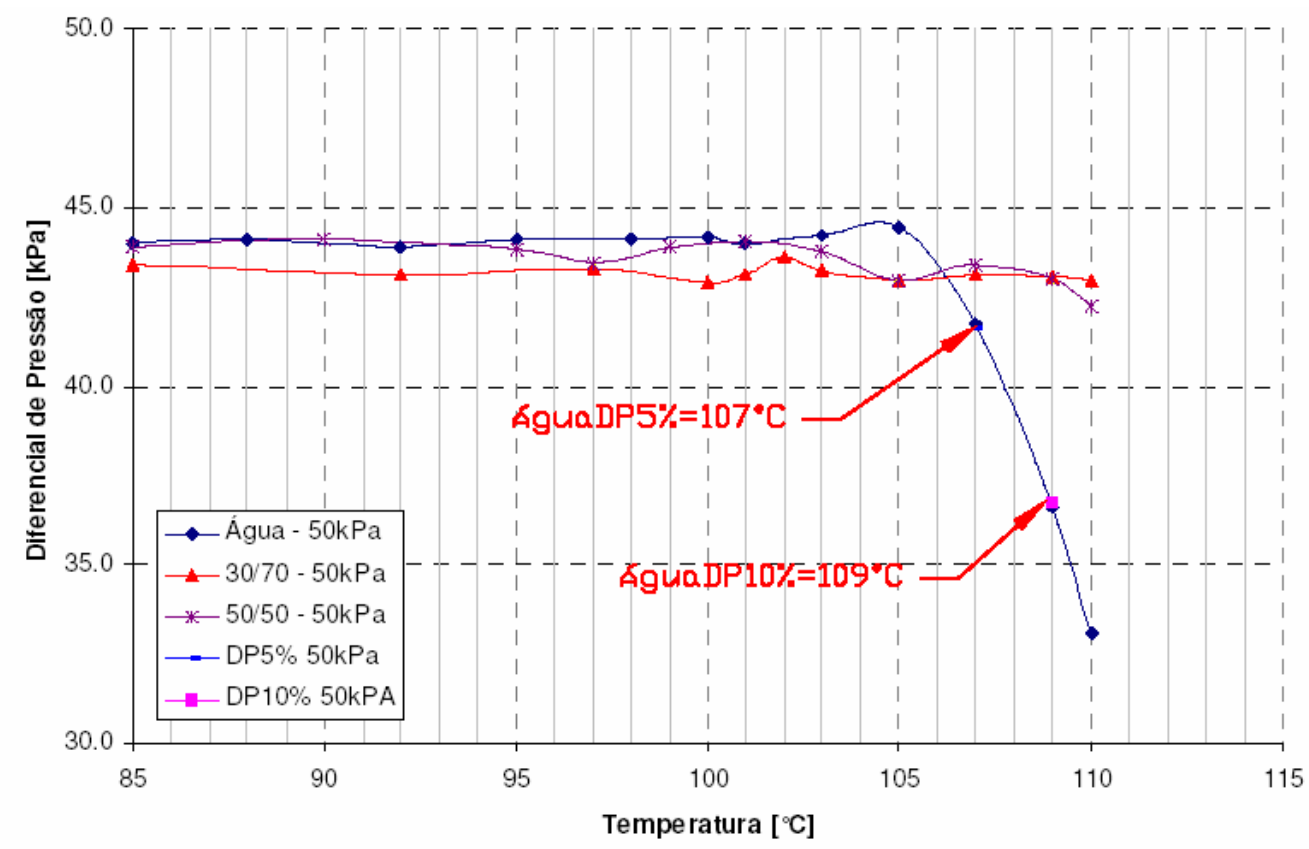

Figura 48 - Bomba a 3000 rpm e sistema com 50 kPa 
A Figura 49 traz a comparação e identificação da temperatura de cavitação para as concentrações de 50\% etilenoglicol e 50\% água, 30\% etilenoglicol e $70 \%$ água, e 100\% água, com a bomba trabalhando a 3000 rpm e o sistema com pressão de $15 \mathrm{kPa}$.

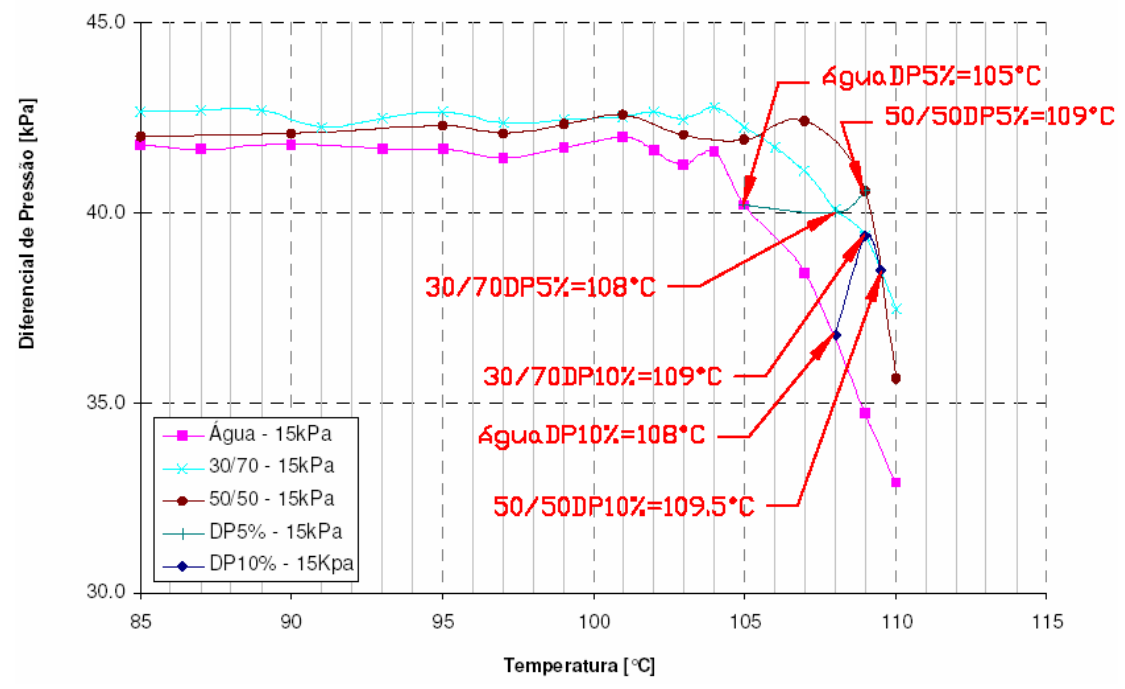

Figura 49 - Bomba a 3000 rpm e sistema com $15 \mathrm{kPa}$ 


\section{CONCLUSÕES}

Este trabalho examina a ocorrência de cavitação em sistemas de arrefecimento, estudando as condições e a intensidade com que o fenômeno ocorre ao se variar os regimes e as circunstâncias de trabalho. O estudo se dá através da medição das variações dos parâmetros de trabalho do sistema de arrefecimento de um veiculo ao trafegar em trânsito urbano e estrada, para então simular essas condições em bancada, de forma a identificar em que condições o fenômeno cavitação ocorre, e também ponderar a influência das variáveis envolvidas no processo.

A simulação é desenvolvida com três diferentes fluidos de arrefecimento:

Mistura 50\% etilenoglicol / 50\% água, mistura 30\% etilenoglicol / 70\% água, e 100\% água. Três fatores têm maior influência na ocorrência do fenômeno cavitação em sistemas de arrefecimento. Eles são a pressão de vapor do fluido bombeado, a temperatura do fluido, e a pressão na entrada da bomba, que varia em função da rotação da bomba.

Os testes em bancada nas três diferentes concentrações da mistura etilenoglicol e água avaliaram fluidos com três diferentes condições de pressão de vapor.

A pressão na entrada foi variada, associando-se diferentes pressões de sistema e diferentes rotações de trabalho da bomba. A determinação dos valores das pressões de sistema para a realização da simulação em bancada se deu através do monitoramento das pressões no vaso de expansão do veiculo testado.

Os resultados dos testes foram ao encontro das conclusões de Hosny (2006), revelando que quão maior for a concentração de etilenoglicol na mistura, menor será a suscetibilidade do sistema à ocorrência de cavitação, 
devido à elevação da temperatura necessária para alcançar a pressão de vapor.

Em contrapartida, constatou-se em todas as misturas testadas, que quão maior for a rotação da bomba, mais propenso à ocorrência de cavitação será o sistema de arrefecimento, devido à diminuição de pressão na entrada da bomba. Dessa forma complementa-se o trabalho de Kim (2000), que avaliou a ocorrência de cavitação para diferentes pressões de sistema, utilizando apenas a mistura de $50 \%$ água e $50 \%$ etilenoglicol.

Ao comparar os resultados das simulações feitas em bancada, expressos no parágrafo 3.3.3. com as medições dos parâmetros de funcionamento, experimentadas no parágrafo 3.1, conclui-se que ao se utilizar a mistura de $50 \%$ etilenoglicol com $50 \%$ água, como fluido de arrefecimento, a cavitação não ocorre mesmo para as rotações mais elevadas, pois a temperatura de cavitação não é alcançada para esta condição.

Com a mistura de $30 \%$ etilenoglicol com $70 \%$ água, a bomba estaria na eminência de ocorrência de cavitação, quando trabalhando a uma rotação igual ou superior a 5000 rpm, e o motor já tenha concluído sua fase de aquecimento.

Para a condição onde água pura é utilizada como fluido de arrefecimento, a bomba cavita para rotações superiores a $4000 \mathrm{rpm}$, na fase de aquecimento do motor, onde a pressão do sistema é menor e a temperatura do fluido chega a algo em torno de $94^{\circ} \mathrm{C}$. Par rotações superiores a $5000 \mathrm{rpm}$ a bomba está em freqüente estado de cavitação para praticamente todas as condições de operação do motor.

A análise das incertezas mostrou que quanto maior for a rotação, menor será a influência da medição da pressão de entrada na incerteza combinada, ou seja, quanto maior a rotação, menor será a incerteza. Ao se avaliar as 
incertezas combinadas, verifica-se a ocorrência de sobreposições entre as faixas onde a temperatura de cavitação é esperada de se encontrar.

A temperatura de cavitação em uma bomba d'água aplicada no sistema de arrefecimento de um motor de combustão interna foi estudada também por Huang (2004), usando três fluidos diferentes: 100\% água, 50\% etilenoglicol e $50 \%$ água, e 100\% etilenoglicol, em diversas rotações de trabalho, o trabalho apresentado aqui apresentado, contribui acrescentando resultados para as misturas $70 \%$ água e $30 \%$ etilenoglicol, e estudando diferentes pressões de trabalho.

Ao correlacionar os resultados dos experimentos realizados neste trabalho, com os resultados de Hattori (2006) e Hosny (2006), que estudaram a influencia da temperatura na intensidade de cavitação, conclui-se que a faixa de temperatura mais crítica em termos de erosão encontra-se entre 50 e $85^{\circ}$ C. Isto indica que um sistema de arrefecimento, no caso de ocorrência de cavitação, estará em uma faixa mais amena, pois mesmo operando na condição mais critica, a 5000 rpm com 100\% de água no sistema, a cavitação só ocorre a partir de $89^{\circ} \mathrm{C}$. 


\section{ANEXO A \\ Valores das incertezas combinadas}

A Tabela A1 mostra o cálculo da incerteza combinada para as medições feitas a $1000 \mathrm{rpm}$, com pressão de sistema de $15 \mathrm{kPa}$, utilizando água como fluido.

Tabela A1 - Incerteza combinada para $1000 \mathrm{rpm}$, água com $15 \mathrm{kPa}$ de pressão de sistema.

\begin{tabular}{|c|c|c|c|c|c|}
\hline Índice & $\mathbf{T}\left[{ }^{\circ} \mathbf{C}\right]$ & $\mathbf{P} 1[\mathbf{k P a}]$ & $\mathbf{P} 2[\mathbf{k P a}]$ & $\mathbf{D P}[\mathbf{k P a}]$ & $\begin{array}{c}\mathbf{u}_{\mathbf{c}} \\
{[\%]}\end{array}$ \\
\hline 1 & 85 & 11,5 & 17,6 & 6,1 & 6,0 \\
\hline 2 & 87 & 11,4 & 17,3 & 5,9 & 6,1 \\
\hline 3 & 89 & 11,6 & 17,3 & 5,7 & 6,3 \\
\hline 4 & 91 & 11,2 & 17,1 & 5,9 & 6,0 \\
\hline 5 & 93 & 11,3 & 17,0 & 5,7 & 6,2 \\
\hline 6 & 95 & 11,7 & 17,4 & 5,7 & 6,4 \\
\hline 7 & 97 & 11,8 & 17,4 & 5,6 & 6,5 \\
\hline 8 & 99 & 10,8 & 16,8 & 6,0 & 5,8 \\
\hline 9 & 101 & 11,5 & 17,4 & 5,9 & 6,2 \\
\hline 10 & 103 & 11,2 & 17,3 & 6,1 & 5,9 \\
\hline 11 & 105 & 11,6 & 17,3 & 5,7 & 6,3 \\
\hline 12 & 107 & 11,2 & 16,9 & 5,7 & 6,2 \\
\hline 13 & 110 & 11,5 & 17,4 & 5,9 & 6,2 \\
\hline
\end{tabular}

As Tabelas A2 e A3 apresentam o cálculo dos coeficientes de sensibilidade das medições das pressões de entrada e saída, respectivamente. A primeira coluna referencia a seqüência de medição. $A$ segunda, terceira e quarta coluna contém a informação para cada um dos componentes da Eq. 13. A quinta coluna traz a contribuição para a incerteza combinada. 
Tabela A2 - Coeficientes de sensibilidade para a pressão de entrada a 1000 rpm, água com $15 \mathrm{kPa}$ de pressão de sistema.

\begin{tabular}{|c|c|c|c|c|}
\hline Índice & $\mathbf{S}_{\mathbf{P} \mathbf{1}}$ & $\mathbf{S}_{\mathbf{P} \mathbf{1} \mathbf{x}} \mathbf{u}_{\mathbf{p}}$ & $\begin{array}{c}\mathbf{1 0 0} \mathbf{x}\left(\mathbf{S}_{\mathbf{P} \mathbf{x}}\right. \\
\left.\mathbf{u}_{\mathbf{p}}\right)^{2}\end{array}$ & $\begin{array}{c}\text { Contribuição do } \\
\text { Transdutor de Pressão da } \\
\text { Entrada[\%] }\end{array}$ \\
\hline 1 & $-1,9$ & $-0,3$ & 10,8 & 29,9 \\
\hline 2 & $-1,9$ & $-0,3$ & 11,5 & 30,4 \\
\hline 3 & $-2,0$ & $-0,4$ & 12,4 & 31,0 \\
\hline 4 & $-1,9$ & $-0,3$ & 10,8 & 30,0 \\
\hline 5 & $-2,0$ & $-0,3$ & 11,8 & 30,6 \\
\hline 6 & $-2,1$ & $-0,4$ & 12,8 & 31,1 \\
\hline 7 & $-2,1$ & $-0,4$ & 13,4 & 31,5 \\
\hline 8 & $-1,8$ & $-0,3$ & 10,0 & 29,4 \\
\hline 9 & $-1,9$ & $-0,3$ & 11,5 & 30,4 \\
\hline 10 & $-1,8$ & $-0,3$ & 10,2 & 29,5 \\
\hline 11 & $-2,0$ & $-0,4$ & 12,4 & 31,0 \\
\hline 12 & $-2,0$ & $-0,3$ & 11,6 & 30,5 \\
\hline 13 & $-1,9$ & $-0,3$ & 11,5 & 30,4 \\
\hline
\end{tabular}

Tabela A3 - Coeficientes de sensibilidade para a pressão de saída a 1000 rpm, água com 15 kPa de pressão de sistema.

\begin{tabular}{|c|c|c|c|c|}
\hline Índice & $S_{\mathrm{P} 2}$ & $\mathrm{~S}_{\mathrm{P} 2 \times} \mathrm{u}_{\mathrm{p}}$ & $100 \times\left(\mathrm{S}_{\mathrm{P} 2 \times} \mathrm{u}_{\mathrm{p}}\right)^{2}$ & $\begin{array}{c}\text { Contribuição do Transdutor } \\
\text { de Pressão da Saída [\%] }\end{array}$ \\
\hline 1 & 2,9 & 0,5 & 25,2 & 70,1 \\
\hline 2 & 2,9 & 0,5 & 26,3 & 69,6 \\
\hline 3 & 3,0 & 0,5 & 27,7 & 69,0 \\
\hline 4 & 2,9 & 0,5 & 25,3 & 70,0 \\
\hline 5 & 3,0 & 0,5 & 26,8 & 69,4 \\
\hline 6 & 3,1 & 0,5 & 28,2 & 68,9 \\
\hline 7 & 3,1 & 0,5 & 29,2 & 68,5 \\
\hline 8 & 2,8 & 0,5 & 24,0 & 70,6 \\
\hline 9 & 2,9 & 0,5 & 26,3 & 69,6 \\
\hline 10 & 2,8 & 0,5 & 24,4 & 70,5 \\
\hline 11 & 3,0 & 0,5 & 27,7 & 69,0 \\
\hline 12 & 3,0 & 0,5 & 26,5 & 69,5 \\
\hline 13 & 2,9 & 0,5 & 26,3 & 69,6 \\
\hline
\end{tabular}

A Tabela A4 trás o cálculo da incerteza combinada para as medições feitas a 1000 rpm, com pressão de sistema de $15 \mathrm{kPa}$, utilizando água como fluido. 
Tabela A4 - Incerteza combinada para $2000 \mathrm{rpm}$, água com $15 \mathrm{kPa}$ de pressão de sistema.

\begin{tabular}{|c|c|c|c|c|c|}
\hline Índice & $\mathbf{T}\left[{ }^{\circ} \mathbf{C}\right]$ & $\mathbf{P 1}[\mathbf{k P a}]$ & $\mathbf{P 2}[\mathbf{k P a}]$ & $\begin{array}{c}\mathbf{D P} \\
{[\mathbf{k P a}]}\end{array}$ & $\begin{array}{c}\mathbf{u}_{\mathbf{c}} \\
{[\%]}\end{array}$ \\
\hline 1 & 85 & 11,3 & 31,3 & 20,0 & 2,9 \\
\hline 2 & 87 & 11,0 & 31,3 & 20,3 & 2,8 \\
\hline 3 & 89 & 11,2 & 31,5 & 20,3 & 2,9 \\
\hline 4 & 91 & 11,9 & 31,9 & 20,0 & 3,0 \\
\hline 5 & 93 & 11,0 & 31,1 & 20,1 & 2,9 \\
\hline 6 & 95 & 11,2 & 31,5 & 20,3 & 2,9 \\
\hline 7 & 97 & 11,7 & 31,9 & 20,2 & 2,9 \\
\hline 8 & 99 & 11,0 & 31,3 & 20,3 & 2,8 \\
\hline 9 & 101 & 11,2 & 31,4 & 20,2 & 2,9 \\
\hline 10 & 103 & 11,1 & 31,1 & 20,0 & 2,9 \\
\hline 11 & 105 & 11,4 & 31,3 & 19,9 & 2,9 \\
\hline 12 & 107 & 11,3 & 31,3 & 20,0 & 2,9 \\
\hline 13 & 110 & 11,4 & 31,3 & 19,9 & 2,9 \\
\hline
\end{tabular}

As Tabelas A5 e A6 apresentam o cálculo dos coeficientes de sensibilidade das medições das pressões de entrada e saída, respectivamente. A primeira coluna referencia a seqüência de medição. A segunda, terceira e quarta coluna contém a informação para cada um dos componentes da Eq. 13. A quinta coluna traz a contribuição para a incerteza combinada.

Tabela A5 - Coeficientes de sensibilidade para a pressão de entrada a $2000 \mathrm{rpm}$, água com $15 \mathrm{kPa}$ de pressão de sistema.

\begin{tabular}{|c|c|c|c|c|}
\hline Índice & $\mathbf{S}_{\mathbf{P} \mathbf{1}}$ & $\mathbf{S}_{\mathbf{P} \mathbf{1} \mathbf{x}} \mathbf{u}_{\mathbf{p}}$ & $\mathbf{1 0 0} \mathbf{x}\left(\mathbf{S}_{\mathbf{p} \mathbf{x}} \mathbf{\mathbf { u } _ { \mathbf { p } }}\right)^{\mathbf{2}}$ & $\begin{array}{c}\text { Contribuição do Transdutor } \\
\text { de Pressão da Entrada[\%] }\end{array}$ \\
\hline 1 & $-0,6$ & $-0,1$ & 1,0 & 11,5 \\
\hline 2 & $-0,5$ & $-0,1$ & 0,9 & 11,0 \\
\hline 3 & $-0,6$ & $-0,1$ & 0,9 & 11,2 \\
\hline 4 & $-0,6$ & $-0,1$ & 1,1 & 12,2 \\
\hline 5 & $-0,5$ & $-0,1$ & 0,9 & 11,1 \\
\hline 6 & $-0,6$ & $-0,1$ & 0,9 & 11,2 \\
\hline 7 & $-0,6$ & $-0,1$ & 1,0 & 11,9 \\
\hline 8 & $-0,5$ & $-0,1$ & 0,9 & 11,0 \\
\hline 9 & $-0,6$ & $-0,1$ & 0,9 & 11,3 \\
\hline 10 & $-0,6$ & $-0,1$ & 0,9 & 11,3 \\
\hline 11 & $-0,6$ & $-0,1$ & 1,0 & 11,7 \\
\hline 12 & $-0,6$ & $-0,1$ & 1,0 & 11,5 \\
\hline 13 & $-0,6$ & $-0,1$ & 1,0 & 11,7 \\
\hline
\end{tabular}


Tabela A6 - Coeficientes de sensibilidade para a pressão de saída a 2000rpm,água com $15 \mathrm{kPa}$ de pressão de sistema.

\begin{tabular}{|c|c|c|c|c|}
\hline Índice & $\mathbf{S}_{\mathbf{P} \mathbf{2}}$ & $\mathbf{S}_{\mathbf{P} \mathbf{2}} \mathbf{u}_{\mathbf{p}}$ & $\mathbf{1 0 0} \mathbf{x}\left(\mathbf{S}_{\mathbf{P 2} \mathbf{x}} \mathbf{u}_{\mathbf{p}}\right)^{\mathbf{2}}$ & $\begin{array}{c}\text { Contribuição do Transdutor } \\
\text { de Pressão da Saída [\%] }\end{array}$ \\
\hline 1 & 1,6 & 0,3 & 7,4 & 88,5 \\
\hline 2 & 1,5 & 0,3 & 7,2 & 89,0 \\
\hline 3 & 1,6 & 0,3 & 7,3 & 88,8 \\
\hline 4 & 1,6 & 0,3 & 7,7 & 87,8 \\
\hline 5 & 1,5 & 0,3 & 7,3 & 88,9 \\
\hline 6 & 1,6 & 0,3 & 7,3 & 88,8 \\
\hline 7 & 1,6 & 0,3 & 7,6 & 88,1 \\
\hline 8 & 1,5 & 0,3 & 7,2 & 89,0 \\
\hline 9 & 1,6 & 0,3 & 7,3 & 88,7 \\
\hline 10 & 1,6 & 0,3 & 7,3 & 88,7 \\
\hline 11 & 1,6 & 0,3 & 7,5 & 88,5 \\
\hline 12 & 1,6 & 0,3 & 7,4 & 88,3 \\
\hline 13 & 1,6 & 0,3 & 7,5 & \multicolumn{2}{|c|}{} \\
\hline
\end{tabular}

A Tabela A7 trás o cálculo da incerteza combinada para as medições feitas a 3000 rpm, com pressão de sistema de $15 \mathrm{kPa}$, utilizando água como fluido.

Tabela A7 - Incerteza combinada para 3000 rpm, água com $15 \mathrm{kPa}$ de pressão de sistema.

\begin{tabular}{|c|c|c|c|c|c|}
\hline Índice & $\mathbf{T}\left[{ }^{\circ} \mathbf{C}\right]$ & $\mathbf{P} 1[\mathbf{k P a}]$ & $\mathbf{P} 2 \mathbf{[ k P a}]$ & $\mathbf{D P}[\mathbf{k P a}]$ & $\begin{array}{c}\mathbf{u}_{\mathbf{c}} \\
{[\%]}\end{array}$ \\
\hline 1 & 85 & 11,9 & 53,7 & 41,8 & 2,3 \\
\hline 2 & 87 & 11,8 & 53,5 & 41,7 & 2,3 \\
\hline 3 & 89 & 12,1 & 53,9 & 41,8 & 2,3 \\
\hline 4 & 91 & 12,5 & 54,2 & 41,7 & 2,3 \\
\hline 5 & 93 & 11,6 & 53,3 & 41,7 & 2,3 \\
\hline 6 & 95 & 11,8 & 53,3 & 41,5 & 2,3 \\
\hline 7 & 97 & 11,5 & 53,2 & 41,7 & 2,3 \\
\hline 8 & 99 & 11,5 & 53,5 & 42,0 & 2,3 \\
\hline 9 & 101 & 11,6 & 53,3 & 41,7 & 2,3 \\
\hline 10 & 103 & 11,8 & 53,1 & 41,3 & 2,3 \\
\hline 11 & 105 & 11,4 & 53,0 & 41,6 & 2,3 \\
\hline 12 & 107 & 11,2 & 51,4 & 40,2 & 2,3 \\
\hline 13 & 110 & 11,6 & 50,0 & 38,4 & 2,3 \\
\hline 13 & 110 & 11,2 & 46,0 & 34,75 & 2,4 \\
\hline 13 & 110 & 11,4 & 44,3 & 32,9 & 2,4 \\
\hline
\end{tabular}


As Tabelas A8 e A9 apresentam o cálculo dos coeficientes de sensibilidade das medições das pressões de entrada e saída, respectivamente. A primeira coluna referencia a seqüência de medição. A segunda, terceira e quarta coluna contém a informação para cada um dos componentes da Eq. 13. A quinta coluna traz a contribuição para a incerteza combinada.

Tabela A8 - Coeficientes de sensibilidade para a pressão de entrada a 3000 rpm, água com 15 kPa de pressão de sistema.

\begin{tabular}{|c|c|c|c|c|}
\hline Índice & $\mathbf{S}_{\mathbf{P} \mathbf{1}}$ & $\mathbf{S}_{\mathbf{P} \mathbf{1} \mathbf{x}} \mathbf{u}_{\mathbf{p}}$ & $\mathbf{1 0 0} \mathbf{x}\left(\mathbf{S}_{\mathbf{P} \mathbf{x}} \mathbf{\mathbf { u } _ { \mathbf { p } }}\right)$ & $\begin{array}{c}\mathbf{2} \\
\text { Contribuição do Transdutor } \\
\text { de Pressão da Entrada[\%] }\end{array}$ \\
\hline 1 & $-0,3$ & 0,0 & 0,2 & 4,7 \\
\hline 2 & $-0,3$ & 0,0 & 0,2 & 4,6 \\
\hline 3 & $-0,3$ & $-0,1$ & 0,3 & 4,8 \\
\hline 4 & $-0,3$ & $-0,1$ & 0,3 & 5,1 \\
\hline 5 & $-0,3$ & 0,0 & 0,2 & 4,5 \\
\hline 6 & $-0,3$ & 0,0 & 0,2 & 4,7 \\
\hline 7 & $-0,3$ & 0,0 & 0,2 & 4,5 \\
\hline 8 & $-0,3$ & 0,0 & 0,2 & 4,4 \\
\hline 9 & $-0,3$ & 0,0 & 0,2 & 4,5 \\
\hline 10 & $-0,3$ & 0,0 & 0,2 & 4,7 \\
\hline 11 & $-0,3$ & 0,0 & 0,2 & 4,4 \\
\hline 12 & $-0,3$ & 0,0 & 0,2 & 4,5 \\
\hline 13 & $-0,3$ & $-0,1$ & 0,3 & 5,1 \\
\hline 14 & $-0,3$ & $-0,1$ & 0,3 & 5,6 \\
\hline 15 & $-0,3$ & $-0,1$ & 0,4 & 6,2 \\
\hline
\end{tabular}


Tabela A9 - Coeficientes de sensibilidade para a pressão de saída a 3000 rpm, água com $15 \mathrm{kPa}$ de pressão de sistema.

\begin{tabular}{|c|c|c|c|c|}
\hline Índice & $\mathbf{S}_{\mathbf{P} \mathbf{2}}$ & $\mathbf{S}_{\mathbf{P} \mathbf{2} \times} \mathbf{u}_{\mathbf{p}}$ & $\mathbf{1 0 0} \mathbf{x}\left(\mathbf{S}_{\mathbf{P} \mathbf{2}} \mathbf{\mathbf { u } _ { \mathbf { p } }} \mathbf{)}^{\mathbf{2}}\right.$ & $\begin{array}{c}\text { Contribuição do Transdutor } \\
\mathbf{d e ~ P r e s s a ̃ o ~ d a ~ S a i ́ d a ~ [ \% ] ~}\end{array}$ \\
\hline 1 & 1,3 & 0,2 & 5,0 & 95,3 \\
\hline 2 & 1,3 & 0,2 & 5,0 & 95,4 \\
\hline 3 & 1,3 & 0,2 & 5,0 & 95,2 \\
\hline 4 & 1,3 & 0,2 & 5,1 & 94,9 \\
\hline 5 & 1,3 & 0,2 & 4,9 & 95,5 \\
\hline 6 & 1,3 & 0,2 & 5,0 & 95,3 \\
\hline 7 & 1,3 & 0,2 & 4,9 & 95,5 \\
\hline 8 & 1,3 & 0,2 & 4,9 & 95,6 \\
\hline 9 & 1,3 & 0,2 & 4,9 & 95,5 \\
\hline 10 & 1,3 & 0,2 & 5,0 & 95,3 \\
\hline 11 & 1,3 & 0,2 & 4,9 & 95,6 \\
\hline 12 & 1,3 & 0,2 & 4,9 & 95,5 \\
\hline 13 & 1,3 & 0,2 & 5,1 & 94,9 \\
\hline 14 & 1,3 & 0,2 & 5,3 & 94,4 \\
\hline 15 & 1,3 & 0,2 & 5,5 & 93,8 \\
\hline
\end{tabular}

A Tabela A10 trás o cálculo da incerteza combinada para as medições feitas a 4000 rpm, com pressão de sistema de $15 \mathrm{kPa}$, utilizando água como fluido.

Tabela A10 - Incerteza combinada para $4000 \mathrm{rpm}$, água com $15 \mathrm{kPa}$ de pressão de sistema.

\begin{tabular}{|c|c|c|c|c|c|}
\hline Índice & $\mathbf{T}\left[{ }^{\circ} \mathbf{C}\right]$ & $\mathbf{P}$ 1 [kPa] & $\mathbf{P 2}[\mathbf{k P a}]$ & $\mathbf{D P}[\mathbf{k P a}]$ & $\begin{array}{c}\mathbf{u}_{\mathbf{c}} \\
{[\%]}\end{array}$ \\
\hline 1 & 85 & 11,4 & 85,2 & 73,8 & 2,0 \\
\hline 2 & 87 & 11,3 & 84,9 & 73,6 & 2,0 \\
\hline 3 & 89 & 11,5 & 85,3 & 73,8 & 2,0 \\
\hline 4 & 91 & 11,5 & 85,2 & 73,7 & 2,0 \\
\hline 5 & 93 & 11,2 & 84,8 & 73,6 & 2,0 \\
\hline 6 & 95 & 11,4 & 78,3 & 66,9 & 2,1 \\
\hline 7 & 97 & 11,3 & 75,0 & 63,7 & 2,1 \\
\hline 8 & 99 & 11,5 & 70,1 & 58,6 & 2,1 \\
\hline 9 & 101 & 11,2 & 63,8 & 52,6 & 2,1 \\
\hline 10 & 103 & 11,6 & 60,1 & 48,5 & 2,2 \\
\hline 11 & 105 & 11,7 & 58,5 & 46,8 & 2,2 \\
\hline 12 & 107 & 11,5 & 54,2 & 42,7 & 2,3 \\
\hline 13 & 110 & 11,3 & 48,1 & 36,8 & 2,3 \\
\hline
\end{tabular}


As Tabelas A11 e A12 apresentam o cálculo dos coeficientes de sensibilidade das medições das pressões de entrada e saída, respectivamente. A primeira coluna referencia a seqüência de medição. A segunda, terceira e quarta coluna contém a informação para cada um dos componentes da Eq. 13. A quinta coluna traz a contribuição para a incerteza combinada.

Tabela A11 - Coeficientes de sensibilidade para a pressão de entrada a 4000 rpm, água com 15 kPa de pressão de sistema.

\begin{tabular}{|c|c|c|c|c|}
\hline Índice & $S_{p_{1}}$ & $S_{P_{1} x} u_{p}$ & $100 \times\left(S_{p_{1} \times} u_{p}\right)^{2}$ & $\begin{array}{l}\text { Contribuição do } \\
\text { Transdutor de Pressão da } \\
\text { Entrada[\%] }\end{array}$ \\
\hline 1 & $-0,2$ & 0,0 & 0,1 & 1,8 \\
\hline 2 & $-0,2$ & 0,0 & 0,1 & 1,7 \\
\hline 3 & $-0,2$ & 0,0 & 0,1 & 1,8 \\
\hline 4 & $-0,2$ & 0,0 & 0,1 & 1,8 \\
\hline 5 & $-0,2$ & 0,0 & 0,1 & 1,7 \\
\hline 6 & $-0,2$ & 0,0 & 0,1 & 2,1 \\
\hline 7 & $-0,2$ & 0,0 & 0,1 & 2,2 \\
\hline 8 & $-0,2$ & 0,0 & 0,1 & 2,6 \\
\hline 9 & $-0,2$ & 0,0 & 0,1 & 3,0 \\
\hline 10 & $-0,2$ & 0,0 & 0,2 & 3,6 \\
\hline 11 & $-0,3$ & 0,0 & 0,2 & 3,9 \\
\hline 12 & $-0,3$ & 0,0 & 0,2 & 4,3 \\
\hline 13 & $-0,3$ & $-0,1$ & 0,3 & 5,2 \\
\hline
\end{tabular}

Tabela A12 - Coeficientes de sensibilidade para a pressão de saída a 4000 rpm, água com $15 \mathrm{kPa}$ de pressão de sistema.

\begin{tabular}{|c|c|c|c|c|}
\hline Índice & $\mathrm{S}_{\mathrm{P} 2}$ & $S_{P 2 \times} u_{p}$ & $100 \times\left(S_{P 2 \times} u_{p}\right)^{2}$ & $\begin{array}{c}\text { Contribuição do } \\
\text { Transdutor de Pressão da } \\
\text { Saída [\%] }\end{array}$ \\
\hline 1 & 1,2 & 0,2 & 4,0 & 98,2 \\
\hline 2 & 1,2 & 0,2 & 4,0 & 98,3 \\
\hline 3 & 1,2 & 0,2 & 4,0 & 98,2 \\
\hline 4 & 1,2 & 0,2 & 4,0 & 98,2 \\
\hline 5 & 1,2 & 0,2 & 4,0 & 98,3 \\
\hline 6 & 1,2 & 0,2 & 4,1 & 97,9 \\
\hline 7 & 1,2 & 0,2 & 4,2 & 97,8 \\
\hline 8 & 1,2 & 0,2 & 4,3 & 97,4 \\
\hline 9 & 1,2 & 0,2 & 4,5 & 97,0 \\
\hline 10 & 1,2 & 0,2 & 4,6 & 96,4 \\
\hline 11 & 1,3 & 0,2 & 4,7 & 96,1 \\
\hline 12 & 1,3 & 0,2 & 4,9 & 95,7 \\
\hline 13 & 1,3 & 0,2 & 5,2 & 94,8 \\
\hline
\end{tabular}


A Tabela A13 trás o cálculo da incerteza combinada para as medições feitas a 5000 rpm, com pressão de sistema de $15 \mathrm{kPa}$, utilizando água como fluido.

Tabela A13 - Incerteza combinada para $5000 \mathrm{rpm}$, água com $15 \mathrm{kPa}$ de pressão de sistema.

\begin{tabular}{|c|c|c|c|c|c|}
\hline Índice & $\mathbf{T}\left[{ }^{\mathbf{}} \mathbf{C}\right]$ & $\mathbf{P} 1[\mathbf{k P a}]$ & $\mathbf{P} 2[\mathbf{k P a}]$ & $\mathbf{D P}[\mathbf{k P a}]$ & $\begin{array}{c}\mathbf{u}_{\mathbf{c}} \\
{[\%]}\end{array}$ \\
\hline 1 & 85 & 10,5 & 109,4 & 98,9 & 1,9 \\
\hline 2 & 87 & 10,1 & 108,7 & 98,6 & 1,9 \\
\hline 3 & 89 & 10,8 & 109,9 & 99,1 & 1,9 \\
\hline 4 & 91 & 10,9 & 108,6 & 97,7 & 1,9 \\
\hline 5 & 93 & 11,0 & 102,1 & 91,1 & 2,0 \\
\hline 6 & 95 & 10,5 & 96,9 & 86,4 & 2,0 \\
\hline 7 & 97 & 10,6 & 91,4 & 80,8 & 2,0 \\
\hline 8 & 99 & 10,9 & 88,1 & 77,2 & 2,0 \\
\hline 9 & 100 & 11,1 & 83,8 & 72,7 & 2,0 \\
\hline 10 & 103 & 10,9 & 74,3 & 63,4 & 2,1 \\
\hline 11 & 106 & 10,8 & 63,5 & 52,7 & 2,1 \\
\hline 12 & 110 & 11,0 & 52,8 & 41,8 & 2,2 \\
\hline
\end{tabular}

As Tabelas A14 e A15 apresentam o cálculo dos coeficientes de sensibilidade das medições das pressões de entrada e saída, respectivamente. A primeira coluna referencia a seqüência de medição. A segunda, terceira e quarta coluna contém a informação para cada um dos componentes da Eq. 13. A quinta coluna traz a contribuição para a incerteza combinada. 
Tabela A14 - Coeficientes de sensibilidade para a pressão de entrada a 5000 rpm, água com 15 kPa de pressão de sistema.

\begin{tabular}{|c|c|c|c|c|}
\hline Índice & $\mathbf{S}_{\mathbf{P} \mathbf{1}}$ & $\mathbf{S}_{\mathbf{P} \mathbf{x}} \mathbf{u}_{\mathbf{p}}$ & $\mathbf{1 0 0} \mathbf{x}\left(\mathbf{S}_{\mathbf{p} \mathbf{x}} \mathbf{u}_{\mathbf{p}}\right)^{\mathbf{2}}$ & $\begin{array}{c}\text { Contribuição do Transdutor } \\
\text { de Pressão da Entrada[\%] }\end{array}$ \\
\hline 1 & $-0,1$ & 0,0 & 0,0 & 0,9 \\
\hline 2 & $-0,1$ & 0,0 & 0,0 & 0,9 \\
\hline 3 & $-0,1$ & 0,0 & 0,0 & 1,0 \\
\hline 4 & $-0,1$ & 0,0 & 0,0 & 1,0 \\
\hline 5 & $-0,1$ & 0,0 & 0,0 & 1,1 \\
\hline 6 & $-0,1$ & 0,0 & 0,0 & 1,2 \\
\hline 7 & $-0,1$ & 0,0 & 0,1 & 1,3 \\
\hline 8 & $-0,1$ & 0,0 & 0,1 & 1,5 \\
\hline 9 & $-0,2$ & 0,0 & 0,1 & 1,7 \\
\hline 10 & $-0,2$ & 0,0 & 0,1 & 2,1 \\
\hline 11 & $-0,2$ & 0,0 & 0,1 & 2,8 \\
\hline 12 & $-0,3$ & 0,0 & 0,2 & 4,2 \\
\hline
\end{tabular}

Tabela A15 - Coeficientes de sensibilidade para a pressão de saída a 5000 rpm, água com 15 kPa de pressão de sistema.

\begin{tabular}{|c|c|c|c|c|}
\hline Índice & $S_{P 2}$ & $S_{P 2 \times} u_{p}$ & $100 \times\left(S_{P 2 \times} u_{p}\right)^{2}$ & $\begin{array}{l}\text { Contribuição do Transdutor } \\
\text { de Pressão da Saída [\%] }\end{array}$ \\
\hline 1 & 1,1 & 0,2 & 3,7 & 99,1 \\
\hline 2 & 1,1 & 0,2 & 3,7 & 99,1 \\
\hline 3 & 1,1 & 0,2 & 3,7 & 99,0 \\
\hline 4 & 1,1 & 0,2 & 3,7 & 99,0 \\
\hline 5 & 1,1 & 0,2 & 3,8 & 98,9 \\
\hline 6 & 1,1 & 0,2 & 3,8 & 98,8 \\
\hline 7 & 1,1 & 0,2 & 3,9 & 98,7 \\
\hline 8 & 1,1 & 0,2 & 3,9 & 98,5 \\
\hline 9 & 1,2 & 0,2 & 4,0 & 98,3 \\
\hline 10 & 1,2 & 0,2 & 4,2 & 97,9 \\
\hline 11 & 1,2 & 0,2 & 4,4 & 97,2 \\
\hline 12 & 1,3 & 0,2 & 4,8 & 95,8 \\
\hline
\end{tabular}

A Tabela A16 trás o cálculo da incerteza combinada para as medições feitas a 1000 rpm, com pressão de sistema de $50 \mathrm{kPa}$, utilizando água como fluido. 
Tabela A16 - Incerteza combinada para 1000 rpm, água com $50 \mathrm{kPa}$ de pressão de sistema.

\begin{tabular}{|c|c|c|c|c|c|}
\hline Índice & $\mathbf{T}\left[{ }^{\circ} \mathbf{C}\right]$ & $\mathbf{P}$ [ $[\mathbf{k P a}]$ & $\mathbf{P} 2[\mathbf{k P a}]$ & $\mathbf{D P}[\mathbf{k P a}]$ & $\begin{array}{c}\mathbf{u}_{\mathbf{c}} \\
{[\%]}\end{array}$ \\
\hline 1 & 85 & 42,8 & 50,5 & 7,7 & 15,0 \\
\hline 2 & 87 & 42,7 & 50,4 & 7,7 & 14,9 \\
\hline 3 & 89 & 42,9 & 50,5 & 7,6 & 15,2 \\
\hline 4 & 91 & 42,8 & 50,6 & 7,8 & 14,7 \\
\hline 5 & 93 & 42,6 & 50,4 & 7,8 & 14,8 \\
\hline 6 & 95 & 42,7 & 50,3 & 7,6 & 15,1 \\
\hline 7 & 97 & 42,9 & 50,7 & 7,8 & 14,9 \\
\hline 8 & 99 & 43,3 & 50,8 & 7,5 & 15,6 \\
\hline 9 & 101 & 43,5 & 51,0 & 7,5 & 15,6 \\
\hline 10 & 103 & 43,2 & 50,9 & 7,7 & 15,1 \\
\hline 11 & 105 & 43,1 & 50,7 & 7,6 & 15,2 \\
\hline
\end{tabular}

As Tabelas A17 e A18 apresentam o cálculo dos coeficientes de sensibilidade das medições das pressões de entrada e saída, respectivamente. A primeira coluna referencia a seqüência de medição. A segunda, terceira e quarta coluna contém a informação para cada um dos componentes da Eq. 13. A quinta coluna traz a contribuição para a incerteza combinada.

Tabela A17 - Coeficientes de sensibilidade para a pressão de entrada a 1000 rpm, água com 50 kPa de pressão de sistema.

\begin{tabular}{|c|c|c|c|c|}
\hline Índice & $\mathbf{S}_{\mathbf{P} \mathbf{1}}$ & $\mathbf{S}_{\mathbf{P} \mathbf{1} \mathbf{x}} \mathbf{u}_{\mathbf{p}}$ & $\mathbf{1 0 0} \mathbf{x}\left(\mathbf{S}_{\mathbf{p} \mathbf{x} \mathbf{x}} \mathbf{u}_{\mathbf{p}}\right)^{\mathbf{2}}$ & $\begin{array}{c}\text { Contribuição do Transdutor } \\
\text { de Pressão da Entrada[\%] }\end{array}$ \\
\hline 1 & $-5,6$ & $-1,0$ & 93,5 & 41,8 \\
\hline 2 & $-5,5$ & $-1,0$ & 93,1 & 41,8 \\
\hline 3 & $-5,6$ & $-1,0$ & 96,5 & 41,9 \\
\hline 4 & $-5,5$ & $-1,0$ & 90,6 & 41,7 \\
\hline 5 & $-5,5$ & $-1,0$ & 91,5 & 41,7 \\
\hline 6 & $-5,6$ & $-1,0$ & 94,9 & 41,9 \\
\hline 7 & $-5,5$ & $-1,0$ & 92,2 & 41,7 \\
\hline 8 & $-5,8$ & $-1,0$ & 102,3 & 42,1 \\
\hline 9 & $-5,8$ & $-1,0$ & 101,8 & 42,1 \\
\hline 10 & $-5,6$ & $-1,0$ & 95,3 & 41,9 \\
\hline 11 & $-5,7$ & $-1,0$ & 97,4 & 42,0 \\
\hline
\end{tabular}


Tabela A18 - Coeficientes de sensibilidade para a pressão de saída a 1000 rpm, água com 50 kPa de pressão de sistema.

\begin{tabular}{|c|c|c|c|c|}
\hline Índice & $\mathbf{S}_{\mathbf{P} \mathbf{2}}$ & $\mathbf{S}_{\mathbf{P} \mathbf{2}} \mathbf{u}_{\mathbf{p}}$ & $\mathbf{1 0 0} \mathbf{x}\left(\mathbf{S}_{\mathbf{P} \mathbf{x}} \mathbf{\mathbf { u } _ { \mathbf { p } }}\right)$ & $\begin{array}{c}\mathbf{2} \\
\text { Contribuição do Transdutor } \\
\text { de Pressão da Saída [\%] }\end{array}$ \\
\hline 1 & 6,6 & 1,1 & 130,2 & 58,2 \\
\hline 2 & 6,5 & 1,1 & 129,7 & 58,2 \\
\hline 3 & 6,6 & 1,2 & 133,7 & 58,1 \\
\hline 4 & 6,5 & 1,1 & 126,7 & 58,3 \\
\hline 5 & 6,5 & 1,1 & 127,8 & 58,3 \\
\hline 6 & 6,6 & 1,1 & 131,9 & 58,1 \\
\hline 7 & 6,5 & 1,1 & 128,6 & 58,3 \\
\hline 8 & 6,8 & 1,2 & 140,5 & 57,9 \\
\hline 9 & 6,8 & 1,2 & 140,0 & 57,9 \\
\hline 10 & 6,6 & 1,2 & 132,3 & 58,1 \\
\hline 11 & 6,7 & 1,2 & 134,7 & 58,0 \\
\hline
\end{tabular}

A Tabela A19 trás o cálculo da incerteza combinada para as medições feitas a 2000 rpm, com pressão de sistema de $50 \mathrm{kPa}$, utilizando água como fluido.

Tabela 21 - Incerteza combinada para 2000 rpm, água com 50 kPa de pressão de sistema.

\begin{tabular}{|c|c|c|c|c|c|}
\hline Índice & $\mathbf{T}\left[{ }^{\circ} \mathbf{C}\right]$ & $\mathbf{P}$ [ $[\mathbf{k P a}]$ & $\mathbf{P} 2[\mathbf{k P a}]$ & $\mathbf{D P}[\mathbf{k P a}]$ & $\begin{array}{c}\mathbf{u}_{\mathbf{c}} \\
{[\%]}\end{array}$ \\
\hline 1 & 85 & 42,1 & 63,5 & 21,4 & 6,2 \\
\hline 2 & 88 & 42,2 & 63,4 & 21,2 & 6,3 \\
\hline 3 & 92 & 42,1 & 63,6 & 21,5 & 6,2 \\
\hline 4 & 95 & 42,4 & 63,8 & 21,4 & 6,2 \\
\hline 5 & 97 & 42,1 & 63,4 & 21,3 & 6,2 \\
\hline 6 & 99 & 42,7 & 63,6 & 20,9 & 6,4 \\
\hline 7 & 101 & 42,5 & 63,6 & 21,1 & 6,3 \\
\hline 8 & 103 & 42,2 & 63,5 & 21,3 & 6,2 \\
\hline 9 & 105 & 42,3 & 63,6 & 21,3 & 6,2 \\
\hline 10 & 107 & 42,1 & 63,5 & 21,4 & 6,2 \\
\hline 11 & 110 & 42,2 & 63,3 & 21,1 & 6,3 \\
\hline
\end{tabular}

As Tabelas A20 e A21 apresentam o cálculo dos coeficientes de sensibilidade das medições das pressões de entrada e saída, respectivamente. A primeira coluna referencia a seqüência de medição. $A$ segunda, terceira e quarta coluna contém a informação para cada um dos componentes da Eq. 13. A quinta coluna traz a contribuição para a incerteza combinada. 
Tabela A20 - Coeficientes de sensibilidade para a pressão de entrada a 2000 rpm, água com 50 kPa de pressão de sistema.

\begin{tabular}{|c|c|c|c|c|}
\hline Índice & $S_{P 1}$ & $S_{P 1 \times} u_{p}$ & $100 \times\left(S_{P 1} \times u_{p}\right)^{2}$ & $\begin{array}{l}\text { Contribuição do Transdutor } \\
\text { de Pressão da Entrada[\%] }\end{array}$ \\
\hline 1 & $-2,0$ & $-0,3$ & 11,7 & 30,5 \\
\hline 2 & $-2,0$ & $\begin{array}{l}-0,3 \\
\end{array}$ & 12,0 & 30,7 \\
\hline 3 & $-2,0$ & \begin{tabular}{l|l}
$-0,3$ \\
\end{tabular} & 11,6 & 30,5 \\
\hline 4 & $-2,0$ & $-0,3$ & 11,9 & 30,7 \\
\hline 5 & $-2,0$ & $-0,3$ & 11,9 & 30,6 \\
\hline 6 & $-2,0$ & $-0,4$ & 12,6 & 31,1 \\
\hline 7 & $-2,0$ & $-0,4$ & 12,3 & 30,9 \\
\hline 8 & $-2,0$ & \begin{tabular}{|c|}
$-0,3$ \\
\end{tabular} & 11,9 & 30,7 \\
\hline 9 & $-2,0$ & $-0,3$ & 12,0 & 30,7 \\
\hline 10 & $-2,0$ & $-0,3$ & 11,7 & 30,5 \\
\hline 11 & $-2,0$ & $-0,3$ & 12,1 & 30,8 \\
\hline
\end{tabular}

Tabela A21 - Coeficientes de sensibilidade para a pressão de saída a 2000 rpm, água com 50 kPa de pressão de sistema.

\begin{tabular}{|c|c|c|c|c|}
\hline Índice & $\mathbf{S}_{\mathbf{P} \mathbf{2}}$ & $\mathbf{S}_{\mathbf{P} \mathbf{2} \mathbf{x}} \mathbf{u}_{\mathbf{p}}$ & $\mathbf{1 0 0} \mathbf{x}\left(\mathbf{S}_{\mathbf{P 2} \mathbf{x}} \mathbf{u}_{\mathbf{p}}\right)^{\mathbf{2}}$ & $\begin{array}{c}\text { Contribuição do Transdutor } \\
\text { de Pressão da Saída [\%] }\end{array}$ \\
\hline 1 & 3,0 & 0,5 & 26,7 & 69,5 \\
\hline 2 & 3,0 & 0,5 & 27,1 & 69,3 \\
\hline 3 & 3,0 & 0,5 & 26,5 & 69,5 \\
\hline 4 & 3,0 & 0,5 & 27,0 & 69,3 \\
\hline 5 & 3,0 & 0,5 & 26,9 & 69,4 \\
\hline 6 & 3,0 & 0,5 & 28,0 & 68,9 \\
\hline 7 & 3,0 & 0,5 & 27,5 & 69,1 \\
\hline 8 & 3,0 & 0,5 & 27,0 & 69,3 \\
\hline 9 & 3,0 & 0,5 & 27,0 & 69,3 \\
\hline 10 & 3,0 & 0,5 & 26,7 & 69,5 \\
\hline 11 & 3,0 & 0,5 & 27,2 & 69,2 \\
\hline
\end{tabular}

A Tabela 24 trás o cálculo da incerteza combinada para as medições feitas a 3000 rpm, com pressão de sistema de 50 kPa, utilizando água como fluido. 
Tabela A22 - Incerteza combinada para 3000 rpm, água com 50 kPa de pressão de sistema.

\begin{tabular}{|c|c|c|c|c|c|}
\hline Índice & $\mathbf{T}\left[{ }^{\mathbf{C}} \mathbf{C}\right]$ & $\mathbf{P} \mathbf{1}[\mathbf{k P a}]$ & $\mathbf{P} \mathbf{[ \mathbf { k P a } ]}$ & $\mathbf{D P}[\mathbf{k P a}]$ & $\begin{array}{c}\mathbf{u}_{\mathbf{c}} \\
{[\%]}\end{array}$ \\
\hline 1 & 85 & 41,9 & 85,9 & 44,0 & 3,8 \\
\hline 2 & 88 & 41,6 & 85,7 & 44,1 & 3,8 \\
\hline 3 & 92 & 41,7 & 85,6 & 43,9 & 3,8 \\
\hline 4 & 95 & 41,8 & 85,9 & 44,1 & 3,8 \\
\hline 5 & 98 & 41,6 & 85,7 & 44,1 & 3,8 \\
\hline 6 & 100 & 41,7 & 85,9 & 44,2 & 3,8 \\
\hline 7 & 101 & 41,8 & 85,8 & 44,0 & 3,8 \\
\hline 8 & 103 & 42,5 & 86,7 & 44,2 & 3,8 \\
\hline 9 & 105 & 41,9 & 86,3 & 44,4 & 3,8 \\
\hline 10 & 107 & 41,5 & 83,2 & 41,7 & 3,9 \\
\hline 11 & 109 & 41,7 & 78,3 & 36,6 & 4,2 \\
\hline 12 & 110 & 41,8 & 74,9 & 33,1 & 4,5 \\
\hline
\end{tabular}

As Tabelas A23 e A24 apresentam o cálculo dos coeficientes de sensibilidade das medições das pressões de entrada e saída, respectivamente. A primeira coluna referencia a seqüência de medição. A segunda, terceira e quarta coluna contém a informação para cada um dos componentes da Eq. 13. A quinta coluna traz a contribuição para a incerteza combinada.

Tabela A23 - Coeficientes de sensibilidade para a pressão de entrada a 3000 rpm, água com $50 \mathrm{kPa}$ de pressão de sistema.

\begin{tabular}{|c|c|c|c|c|}
\hline Índice & $\mathbf{S}_{\mathbf{p} \mathbf{1}}$ & $\mathbf{S}_{\mathbf{p} \mathbf{x}} \mathbf{\mathbf { u } _ { \mathbf { p } }}$ & $\mathbf{1 0 0} \mathbf{x}\left(\mathbf{S}_{\mathbf{p} \mathbf{x}} \mathbf{\mathbf { u } _ { \mathbf { p } }} \mathbf{~}^{\mathbf{2}}\right.$ & $\begin{array}{c}\text { Contribuição do Transdutor } \\
\mathbf{d e ~ P r e s a ̃ o} \mathbf{d a} \text { Entrada[\%] }\end{array}$ \\
\hline 1 & $-1,0$ & $-0,2$ & 2,7 & 19,2 \\
\hline 2 & $-0,9$ & $-0,2$ & 2,7 & 19,1 \\
\hline 3 & $-0,9$ & $-0,2$ & 2,7 & 19,2 \\
\hline 4 & $-0,9$ & $-0,2$ & 2,7 & 19,1 \\
\hline 5 & $-0,9$ & $-0,2$ & 2,7 & 19,1 \\
\hline 6 & $-0,9$ & $-0,2$ & 2,7 & 19,1 \\
\hline 7 & $-1,0$ & $-0,2$ & 2,7 & 19,2 \\
\hline 8 & $-1,0$ & $-0,2$ & 2,8 & 19,4 \\
\hline 9 & $-0,9$ & $-0,2$ & 2,7 & 19,1 \\
\hline 10 & $-1,0$ & $-0,2$ & 3,0 & 19,9 \\
\hline 11 & $-1,1$ & $-0,2$ & 3,9 & 22,1 \\
\hline 12 & $-1,3$ & $-0,2$ & 4,8 & 23,7 \\
\hline
\end{tabular}


Tabela A24 - Coeficientes de sensibilidade para a pressão de saída a 3000 rpm, água com 50 kPa de pressão de sistema.

\begin{tabular}{|c|c|c|c|c|}
\hline Índice & $S_{P 2}$ & $S_{P 2 \times} u_{p}$ & $100 \times\left(S_{P 2} \times u_{p}\right)^{2}$ & $\begin{array}{l}\text { Contribuição do Transdutor } \\
\text { de Pressão da Saída [\%] }\end{array}$ \\
\hline 1 & 2,0 & 0,3 & 11,5 & 80,8 \\
\hline 2 & 1,9 & 0,3 & 11,4 & 80,9 \\
\hline 3 & 1,9 & 0,3 & 11,5 & 80,8 \\
\hline 4 & 1,9 & 0,3 & 11,5 & 80,9 \\
\hline 5 & 1,9 & 0,3 & 11,4 & 80,9 \\
\hline 6 & 1,9 & 0,3 & 11,4 & 80,9 \\
\hline 7 & 2,0 & 0,3 & 11,5 & 80,8 \\
\hline 8 & 2,0 & 0,3 & 11,6 & 80,6 \\
\hline 9 & 1,9 & 0,3 & 11,4 & 80,9 \\
\hline 10 & 2,0 & 0,3 & 12,0 & 80,1 \\
\hline 11 & 2,1 & 0,4 & 13,9 & 77,9 \\
\hline 12 & 2,3 & 0,4 & 15,5 & 76,3 \\
\hline
\end{tabular}

A Tabela A25 trás o cálculo da incerteza combinada para as medições feitas a 4000 rpm, com pressão de sistema de 50 kPa, utilizando água como fluido.

Tabela A25 - Incerteza combinada para 4000 rpm, água com 50 kPa de pressão de sistema.

\begin{tabular}{|c|c|c|c|c|c|}
\hline Índice & $\mathbf{T}\left[{ }^{\mathbf{C}} \mathbf{C}\right]$ & $\mathbf{P} \mathbf{[}[\mathbf{k P a}]$ & $\mathbf{P} \mathbf{2}[\mathbf{k P a}]$ & $\mathbf{D P}[\mathbf{k P a}]$ & $\begin{array}{c}\mathbf{u}_{\mathbf{c}} \\
{[\%]}\end{array}$ \\
\hline 1 & 85 & 41,1 & 116,6 & 75,5 & 2,8 \\
\hline 2 & 88 & 41,1 & 116,7 & 75,6 & 2,8 \\
\hline 3 & 92 & 41,2 & 116,6 & 75,4 & 2,9 \\
\hline 4 & 95 & 41,0 & 116,5 & 75,5 & 2,8 \\
\hline 5 & 97 & 41,1 & 116,3 & 75,2 & 2,9 \\
\hline 6 & 99 & 41,6 & 117,0 & 75,4 & 2,9 \\
\hline 7 & 101 & 41,5 & 115,8 & 74,3 & 2,9 \\
\hline 8 & 103 & 41,2 & 113,5 & 72,3 & 2,9 \\
\hline 9 & 105 & 41,6 & 112,3 & 70,7 & 2,9 \\
\hline 10 & 107 & 41,5 & 108,7 & 67,2 & 3,0 \\
\hline 11 & 109 & 41,7 & 106,4 & 64,7 & 3,1 \\
\hline 12 & 110 & 41,6 & 103,2 & 61,6 & 3,1 \\
\hline
\end{tabular}

As Tabelas A26 e A27 apresentam o cálculo dos coeficientes de sensibilidade das medições das pressões de entrada e saída, respectivamente. A primeira coluna referencia a seqüência de medição. A 
segunda, terceira e quarta coluna contém a informação para cada um dos componentes da Eq. 13. A quinta coluna traz a contribuição para a incerteza combinada.

Tabela A26 - Coeficientes de sensibilidade para a pressão de entrada a 4000 rpm, água com $50 \mathrm{kPa}$ de pressão de sistema.

\begin{tabular}{|c|c|c|c|c|}
\hline Índice & $\mathbf{S}_{\mathbf{P} \mathbf{1}}$ & $\mathbf{S}_{\mathbf{P} \mathbf{x}} \mathbf{u}_{\mathbf{p}}$ & $\mathbf{1 0 0} \mathbf{x}\left(\mathbf{S}_{\mathbf{P} \mathbf{x}} \mathbf{u}_{\mathbf{p}}\right)^{\mathbf{2}}$ & $\begin{array}{c}\text { Contribuição do Transdutor } \\
\text { de Pressão da Entrada[\%] }\end{array}$ \\
\hline 1 & $-0,5$ & $-0,1$ & 0,9 & 11,1 \\
\hline 2 & $-0,5$ & $-0,1$ & 0,9 & 11,0 \\
\hline 3 & $-0,5$ & $-0,1$ & 0,9 & 11,1 \\
\hline 4 & $-0,5$ & $-0,1$ & 0,9 & 11,0 \\
\hline 5 & $-0,5$ & $-0,1$ & 0,9 & 11,1 \\
\hline 6 & $-0,6$ & $-0,1$ & 0,9 & 11,2 \\
\hline 7 & $-0,6$ & $-0,1$ & 0,9 & 11,4 \\
\hline 8 & $-0,6$ & $-0,1$ & 1,0 & 11,7 \\
\hline 9 & $-0,6$ & $-0,1$ & 1,0 & 12,1 \\
\hline 10 & $-0,6$ & $-0,1$ & 1,2 & 12,7 \\
\hline 11 & $-0,6$ & $-0,1$ & 1,3 & 13,3 \\
\hline 12 & $-0,7$ & $-0,1$ & 1,4 & 14,0 \\
\hline
\end{tabular}

Tabela A7 - Coeficientes de sensibilidade para a pressão de saída a 4000 rpm, água com $50 \mathrm{kPa}$ de pressão de sistema.

\begin{tabular}{|c|c|c|c|c|}
\hline Índice & $S_{P 2}$ & $S_{P 2} \times u_{p}$ & $100 \times\left(S_{P 2} \times u_{p}\right)^{2}$ & $\begin{array}{l}\text { Contribuição do Transdutor } \\
\text { de Pressão da Saída [\%] }\end{array}$ \\
\hline 1 & 1,5 & 0,3 & 7,2 & 88,9 \\
\hline 2 & 1,5 & 0,3 & 7,2 & 89,0 \\
\hline 3 & 1,5 & 0,3 & 7,2 & 88,9 \\
\hline 4 & 1,5 & 0,3 & 7,2 & 89,0 \\
\hline 5 & 1,5 & 0,3 & 7,2 & 88,9 \\
\hline 6 & 1,6 & 0,3 & 7,3 & 88,8 \\
\hline 7 & 1,6 & 0,3 & 7,4 & 88,6 \\
\hline 8 & 1,6 & 0,3 & 7,5 & 88,3 \\
\hline 9 & 1,6 & 0,3 & 7,6 & 87,9 \\
\hline 10 & 1,6 & 0,3 & 7,9 & 87,3 \\
\hline 11 & 1,6 & 0,3 & 8,2 & 86,7 \\
\hline 12 & 1,7 & 0,3 & 8,5 & 86,0 \\
\hline
\end{tabular}

A Tabela A28 trás o cálculo da incerteza combinada para as medições feitas a 5000 rpm, com pressão de sistema de $50 \mathrm{kPa}$, utilizando água como fluido. 
Tabela A28 - Incerteza combinada para 5000 rpm, água com $50 \mathrm{kPa}$ de pressão de sistema.

\begin{tabular}{|c|c|c|c|c|c|}
\hline Índice & $\mathbf{T}\left[{ }^{\circ} \mathbf{C}\right]$ & $\mathbf{P 1}[\mathbf{k P a}]$ & $\mathbf{P} 2[\mathbf{k P a}]$ & $\begin{array}{c}\mathbf{D P} \\
{[\mathbf{k P a}]}\end{array}$ & $\begin{array}{c}\mathbf{u}_{\mathbf{c}} \\
{[\%]}\end{array}$ \\
\hline 1 & 85 & 42,3 & 142,5 & 100,2 & 2,6 \\
\hline 2 & 88 & 42,4 & 142,5 & 100,1 & 2,6 \\
\hline 3 & 91 & 42,1 & 141,9 & 99,8 & 2,6 \\
\hline 4 & 93 & 42,2 & 142,3 & 100,1 & 2,6 \\
\hline 5 & 95 & 41,9 & 139,2 & 97,3 & 2,6 \\
\hline 6 & 97 & 42,8 & 138,5 & 95,7 & 2,6 \\
\hline 7 & 99 & 42,1 & 134,6 & 92,5 & 2,7 \\
\hline 8 & 101 & 41,8 & 132,8 & 91,0 & 2,7 \\
\hline 9 & 103 & 42,3 & 131,4 & 89,1 & 2,7 \\
\hline 10 & 105 & 41,6 & 125,2 & 83,6 & 2,7 \\
\hline 11 & 107 & 41,9 & 121,3 & 79,4 & 2,8 \\
\hline 12 & 110 & 41,7 & 113,6 & 71,9 & 2,9 \\
\hline
\end{tabular}

As Tabelas A29 e A30 apresentam o cálculo dos coeficientes de sensibilidade das medições das pressões de entrada e saída, respectivamente. A primeira coluna referencia a seqüência de medição. A segunda, terceira e quarta coluna contém a informação para cada um dos componentes da Eq. 13. A quinta coluna traz a contribuição para a incerteza combinada.

Tabela A29 - Coeficientes de sensibilidade para a pressão de entrada a 5000 rpm, água com 50 kPa de pressão de sistema.

\begin{tabular}{|c|c|c|c|c|}
\hline Índice & $\mathbf{S}_{\mathbf{P} \mathbf{1}}$ & $\mathbf{S}_{\mathbf{p} \mathbf{1} \mathbf{x}} \mathbf{u}_{\mathbf{p}}$ & $\mathbf{1 0 0} \mathbf{x}\left(\mathbf{S}_{\mathbf{p} \mathbf{x} \mathbf{\mathbf { u }}} \mathbf{u}_{\mathbf{p}} \mathbf{2}^{\mathbf{2}}\right.$ & $\begin{array}{c}\text { Contribuição do Transdutor } \\
\text { de Pressão da Entrada[\%] }\end{array}$ \\
\hline 1 & $-0,4$ & $-0,1$ & 0,5 & 8,1 \\
\hline 2 & $-0,4$ & $-0,1$ & 0,5 & 8,1 \\
\hline 3 & $-0,4$ & $-0,1$ & 0,5 & 8,1 \\
\hline 4 & $-0,4$ & $-0,1$ & 0,5 & 8,1 \\
\hline 5 & $-0,4$ & $-0,1$ & 0,6 & 8,3 \\
\hline 6 & $-0,4$ & $-0,1$ & 0,6 & 8,7 \\
\hline 7 & $-0,5$ & $-0,1$ & 0,6 & 8,9 \\
\hline 8 & $-0,5$ & $-0,1$ & 0,6 & 9,0 \\
\hline 9 & $-0,5$ & $-0,1$ & 0,7 & 9,4 \\
\hline 10 & $-0,5$ & $-0,1$ & 0,7 & 9,9 \\
\hline 11 & $-0,5$ & $-0,1$ & 0,8 & 10,7 \\
\hline 12 & $-0,6$ & $-0,1$ & 1,0 & 11,9 \\
\hline
\end{tabular}


Tabela A30 - Coeficientes de sensibilidade para a pressão de saída a 5000 rpm, água com 50 kPa de pressão de sistema.

\begin{tabular}{|c|c|c|c|c|}
\hline Índice & $S_{P 2}$ & $S_{P 2 \times} u_{p}$ & $100 \times\left(S_{p 2 \times} u_{p}\right)^{2}$ & $\begin{array}{c}\text { Contribuição do } \\
\text { Transdutor de Pressão da } \\
\text { Saída [\%] }\end{array}$ \\
\hline 1 & 1,4 & 0,2 & 6,1 & 91,9 \\
\hline 2 & 1,4 & 0,2 & 6,1 & 91,9 \\
\hline 3 & 1,4 & 0,2 & 6,1 & 91,9 \\
\hline 4 & 1,4 & 0,2 & 6,1 & 91,9 \\
\hline 5 & 1,4 & 0,2 & 6,2 & 91,7 \\
\hline 6 & 1,4 & 0,3 & 6,3 & 91,3 \\
\hline 7 & 1,5 & 0,3 & 6,4 & 91,1 \\
\hline 8 & 1,5 & 0,3 & 6,5 & 91,0 \\
\hline 9 & 1,5 & 0,3 & 6,6 & 90,6 \\
\hline 10 & 1,5 & 0,3 & 6,8 & 90,1 \\
\hline 11 & 1,5 & 0,3 & 7,1 & 89,3 \\
\hline 12 & 1,6 & 0,3 & 7,6 & 88,1 \\
\hline
\end{tabular}

A Tabela A31 trás o cálculo da incerteza combinada para as medições feitas a 1000 rpm, com pressão de sistema de $15 \mathrm{kPa}$, utilizando a mistura de $30 \%$ etilenoglicol e $70 \%$ água como fluido.

Tabela A31 - Incerteza combinada para 1000 rpm, mistura 30/70 com 15 kPa de pressão de sistema.

\begin{tabular}{|c|c|c|c|c|c|}
\hline Índice & $\mathbf{T}\left[{ }^{\circ} \mathbf{C}\right]$ & $\mathbf{P} 1[\mathbf{k P a}]$ & $\mathbf{P} 2 \mathbf{[ k P a}]$ & $\begin{array}{c}\mathbf{D P} \\
{[\mathbf{k P a}]}\end{array}$ & $\begin{array}{c}\mathbf{u}_{\mathbf{c}} \\
{[\%]}\end{array}$ \\
\hline 1 & 85 & 11,4 & 17,6 & 6,2 & 5,9 \\
\hline 2 & 87 & 12,2 & 18,4 & 6,2 & 6,2 \\
\hline 3 & 89 & 12,8 & 19,1 & 6,3 & 6,4 \\
\hline 4 & 91 & 12,4 & 18,8 & 6,4 & 6,2 \\
\hline 5 & 93 & 12,3 & 18,5 & 6,2 & 6,3 \\
\hline 6 & 95 & 12,2 & 18,4 & 6,2 & 6,2 \\
\hline 7 & 97 & 12,8 & 18,8 & 6,0 & 6,6 \\
\hline 8 & 99 & 12,3 & 18,5 & 6,2 & 6,3 \\
\hline 9 & 101 & 11,7 & 18,0 & 6,3 & 5,9 \\
\hline 10 & 102 & 12,8 & 19,2 & 6,4 & 6,3 \\
\hline 11 & 103 & 12,6 & 19,0 & 6,4 & 6,2 \\
\hline 12 & 104 & 13,0 & 19,5 & 6,5 & 6,3 \\
\hline 13 & 105 & 12,9 & 19,1 & 6,2 & 6,4 \\
\hline 14 & 106 & 11,8 & 18,1 & 6,3 & 6,0 \\
\hline 15 & 107 & 12,1 & 18,3 & 6,2 & 6,2 \\
\hline 16 & 108 & 12,8 & 19,0 & 6,2 & 6,5 \\
\hline 17 & 109 & 13,1 & 19,5 & 6,4 & 6,4 \\
\hline 18 & 110 & 12,9 & 19,4 & 6,5 & 6,2 \\
\hline
\end{tabular}


As Tabelas A32 e A33 apresentam o cálculo dos coeficientes de sensibilidade das medições das pressões de entrada e saída, respectivamente. A primeira coluna referencia a seqüência de medição. A segunda, terceira e quarta coluna contém a informação para cada um dos componentes da Eq. 13. A quinta coluna traz a contribuição para a incerteza combinada.

Tabela A32 - Coeficientes de sensibilidade para a pressão de entrada a $1000 \mathrm{rpm}$, mistura 30/70 com $15 \mathrm{kPa}$ de pressão de sistema.

\begin{tabular}{|c|c|c|c|c|}
\hline Índice & $\mathbf{S}_{\mathbf{P} \mathbf{1}}$ & $\mathbf{S}_{\mathbf{P} \mathbf{1} \mathbf{x}} \mathbf{u}_{\mathbf{p}}$ & $\mathbf{1 0 0} \mathbf{x}\left(\mathbf{S}_{\mathbf{p} \mathbf{x}} \mathbf{\mathbf { u } _ { \mathbf { p } }} \mathbf{)}^{\mathbf{2}}\right.$ & $\begin{array}{c}\text { Contribuição do Transdutor } \\
\mathbf{d e ~ P r e s s a ̃ o} \mathbf{d a} \text { Entrada[\%] }\end{array}$ \\
\hline 1 & $-1,8$ & $-0,3$ & 10,2 & 29,6 \\
\hline 2 & $-2,0$ & $-0,3$ & 11,8 & 30,6 \\
\hline 3 & $-2,0$ & $-0,4$ & 12,7 & 31,1 \\
\hline 4 & $-2,0$ & $-0,3$ & 11,5 & 30,4 \\
\hline 5 & $-2,0$ & $-0,3$ & 12,0 & 30,7 \\
\hline 6 & $-2,0$ & $-0,3$ & 11,9 & 30,7 \\
\hline 7 & $-2,1$ & $-0,4$ & 13,9 & 31,7 \\
\hline 8 & $-2,0$ & $-0,3$ & 12,1 & 30,8 \\
\hline 9 & $-1,8$ & $-0,3$ & 10,4 & 29,6 \\
\hline 10 & $-2,0$ & $-0,4$ & 12,3 & 30,9 \\
\hline 11 & $-2,0$ & $-0,3$ & 11,9 & 30,7 \\
\hline 12 & $-2,0$ & $-0,3$ & 12,2 & 30,8 \\
\hline 13 & $-2,1$ & $-0,4$ & 13,0 & 31,3 \\
\hline 14 & $-1,9$ & $-0,3$ & 10,8 & 29,9 \\
\hline 15 & $-2,0$ & $-0,3$ & 11,6 & 30,5 \\
\hline 16 & $-2,1$ & $-0,4$ & 13,1 & 31,3 \\
\hline 17 & $-2,0$ & $-0,4$ & 12,7 & 31,1 \\
\hline 18 & $-2,0$ & $-0,3$ & 11,9 & 30,7 \\
\hline
\end{tabular}


Tabela A33 - Coeficientes de sensibilidade para a pressão de saída a 1000 rpm, mistura 30/70 com 15 kPa de pressão de sistema.

\begin{tabular}{|c|c|c|c|c|}
\hline Índice & $\mathbf{S}_{\mathbf{P} \mathbf{2}}$ & $\mathbf{S}_{\mathbf{P} \mathbf{2}} \mathbf{u}_{\mathbf{p}}$ & $\begin{array}{c}\mathbf{1 0 0} \mathbf{x}\left(\mathbf{S}_{\mathbf{P 2} \mathbf{x}}\right. \\
\mathbf{u}_{\mathbf{p}} \mathbf{2}^{\mathbf{2}}\end{array}$ & $\begin{array}{c}\text { Contribuição do } \\
\text { Transdutor de Pressão } \\
\mathbf{d a} \text { Saída [\%] }\end{array}$ \\
\hline 1 & 2,8 & 0,5 & 24,4 & 70,4 \\
\hline 2 & 3,0 & 0,5 & 26,8 & 69,4 \\
\hline 3 & 3,0 & 0,5 & 28,1 & 68,9 \\
\hline 4 & 3,0 & 0,5 & 26,4 & 69,6 \\
\hline 5 & 3,0 & 0,5 & 27,1 & 69,3 \\
\hline 6 & 3,0 & 0,5 & 27,0 & 69,3 \\
\hline 7 & 3,1 & 0,5 & 29,9 & 68,3 \\
\hline 8 & 3,0 & 0,5 & 27,2 & 69,2 \\
\hline 9 & 2,8 & 0,5 & 24,6 & 60,4 \\
\hline 10 & 3,0 & 0,5 & 27,5 & 69,1 \\
\hline 11 & 3,0 & 0,5 & 27,0 & 69,2 \\
\hline 12 & 3,0 & 0,5 & 27,4 & 68,7 \\
\hline 13 & 3,1 & 0,5 & 28,6 & 70,1 \\
\hline 14 & 2,9 & 0,5 & 25,3 & 69,5 \\
\hline 15 & 3,0 & 0,5 & 26,5 & 68,7 \\
\hline 16 & 3,1 & 0,5 & 28,7 & 68,9 \\
\hline 17 & 3,0 & 0,5 & 28,1 & 69,3 \\
\hline 18 & 3,0 & 0,5 & 27,0 & \\
\hline & & & & \\
\hline
\end{tabular}

A Tabela A34 trás o cálculo da incerteza combinada para as medições feitas a 2000 rpm, com pressão de sistema de $15 \mathrm{kPa}$, utilizando a mistura de 30\% etilenoglicol e $70 \%$ água como fluido. 
Tabela A34 - Incerteza combinada para 2000 rpm, mistura 30/70 com 15 kPa de pressão de sistema.

\begin{tabular}{|c|c|c|c|c|c|}
\hline Índice & $\mathbf{T}\left[{ }^{\circ} \mathbf{C}\right]$ & $\mathbf{P}$ 1 $[\mathbf{k P a}]$ & $\mathbf{P} 2 \mathbf{[ k P a}]$ & $\begin{array}{c}\mathbf{D P} \\
{[\mathbf{k P a}]}\end{array}$ & $\begin{array}{c}\mathbf{U}_{\mathbf{c}} \\
{[\%]}\end{array}$ \\
\hline 1 & 85 & 11,8 & 31,7 & 19,9 & 3,0 \\
\hline 2 & 87 & 11,5 & 31,6 & 20,1 & 2,9 \\
\hline 3 & 89 & 11,2 & 31,4 & 20,2 & 2,9 \\
\hline 4 & 91 & 12,0 & 32,0 & 20,0 & 3,0 \\
\hline 5 & 93 & 10,3 & 30,7 & 20,4 & 2,8 \\
\hline 6 & 95 & 10,2 & 30,4 & 20,2 & 2,8 \\
\hline 7 & 97 & 11,0 & 31,2 & 20,2 & 2,8 \\
\hline 8 & 99 & 10,3 & 30,8 & 20,5 & 2,8 \\
\hline 9 & 101 & 10,1 & 30,5 & 20,4 & 2,7 \\
\hline 10 & 102 & 10,8 & 31,3 & 20,5 & 2,8 \\
\hline 11 & 103 & 10,9 & 31,1 & 20,2 & 2,8 \\
\hline 12 & 104 & 10,8 & 31,2 & 20,4 & 2,8 \\
\hline 13 & 105 & 10,2 & 30,7 & 20,5 & 2,7 \\
\hline 14 & 106 & 9,2 & 29,8 & 20,6 & 2,6 \\
\hline 15 & 107 & 11,1 & 31,1 & 20,0 & 2,9 \\
\hline 16 & 108 & 10,8 & 31,0 & 20,2 & 2,8 \\
\hline 17 & 109 & 11,0 & 31,2 & 20,2 & 2,8 \\
\hline 18 & 110 & 10,9 & 30,7 & 19,8 & 2,9 \\
\hline
\end{tabular}

As Tabelas A35 e A36 apresentam o cálculo dos coeficientes de sensibilidade das medições das pressões de entrada e saída, respectivamente. A primeira coluna referencia a seqüência de medição. A segunda, terceira e quarta coluna contém a informação para cada um dos componentes da Eq. 13. A quinta coluna traz a contribuição para a incerteza combinada. 
Tabela A35 - Coeficientes de sensibilidade para a pressão de entrada a 2000 rpm, mistura 30/70 com 15 kPa de pressão de sistema.

\begin{tabular}{|c|c|c|c|c|}
\hline Índice & $\mathbf{S}_{\mathbf{P} \mathbf{1}}$ & $\mathbf{S}_{\mathbf{P} 1 \mathbf{x}} \mathbf{u}_{\mathbf{p}}$ & $\mathbf{1 0 0} \mathbf{x}\left(\mathbf{S}_{\mathbf{P} \mathbf{1} \mathbf{x}} \mathbf{u}_{\mathbf{p}}\right)^{\mathbf{2}}$ & $\begin{array}{c}\text { Contribuição do Transdutor } \\
\text { de Pressão da Entrada[\%] }\end{array}$ \\
\hline 1 & $-0,6$ & $-0,1$ & 1,1 & 12,2 \\
\hline 2 & $-0,6$ & $-0,1$ & 1,0 & 11,7 \\
\hline 3 & $-0,6$ & $-0,1$ & 0,9 & 11,3 \\
\hline 4 & $-0,6$ & $-0,1$ & 1,1 & 12,3 \\
\hline 5 & $-0,5$ & $-0,1$ & 0,8 & 10,1 \\
\hline 6 & $-0,5$ & $-0,1$ & 0,8 & 10,1 \\
\hline 7 & $-0,5$ & $-0,1$ & 0,9 & 11,0 \\
\hline 8 & $-0,5$ & $-0,1$ & 0,8 & 10,1 \\
\hline 9 & $-0,5$ & $-0,1$ & 0,7 & 9,9 \\
\hline 10 & $-0,5$ & $-0,1$ & 0,8 & 10,7 \\
\hline 11 & $-0,5$ & $-0,1$ & 0,9 & 11,0 \\
\hline 12 & $-0,5$ & $-0,1$ & 0,9 & 10,7 \\
\hline 13 & $-0,5$ & $-0,1$ & 0,7 & 9,9 \\
\hline 14 & $-0,4$ & $-0,1$ & 0,6 & 8,7 \\
\hline 15 & $-0,6$ & $-0,1$ & 0,9 & 11,3 \\
\hline 16 & $-0,5$ & $-0,1$ & 0,9 & 10,9 \\
\hline 17 & $-0,5$ & $-0,1$ & 0,9 & 11,1 \\
\hline 18 & $-0,5$ & $-0,1$ & 0,9 & 11,2 \\
\hline
\end{tabular}

Tabela A36 - Coeficientes de sensibilidade para a pressão de saída a 2000 rpm, mistura 30/70 com 15 kPa de pressão de sistema.

\begin{tabular}{|c|c|c|c|c|}
\hline Índice & $\mathbf{S}_{\mathbf{P} \mathbf{2}}$ & $\mathbf{S}_{\mathbf{P} \mathbf{2} \mathbf{x}} \mathbf{u}_{\mathbf{p}}$ & $\mathbf{1 0 0} \mathbf{x}\left(\mathbf{S}_{\mathbf{P} \mathbf{x}} \mathbf{\mathbf { u } _ { \mathbf { p } }} \mathbf{)}^{\mathbf{2}}\right.$ & $\begin{array}{c}\text { Contribuição do Transdutor } \\
\mathbf{d e ~ P r e s s a ̃ o ~ d a ~ S a i ́ d a ~ [ \% ] ~}\end{array}$ \\
\hline 1 & 1,6 & 0,3 & 7,7 & 87,8 \\
\hline 2 & 1,6 & 0,3 & 7,5 & 88,3 \\
\hline 3 & 1,6 & 0,3 & 7,3 & 88,7 \\
\hline 4 & 1,6 & 0,3 & 7,7 & 87,7 \\
\hline 5 & 1,5 & 0,3 & 6,9 & 89,9 \\
\hline 6 & 1,5 & 0,3 & 6,9 & 89,9 \\
\hline 7 & 1,5 & 0,3 & 7,2 & 89,0 \\
\hline 8 & 1,5 & 0,3 & 6,8 & 89,9 \\
\hline 9 & 1,5 & 0,3 & 6,8 & 90,1 \\
\hline 10 & 1,5 & 0,3 & 7,1 & 89,3 \\
\hline 11 & 1,5 & 0,3 & 7,2 & 89,0 \\
\hline 12 & 1,5 & 0,3 & 7,1 & 89,3 \\
\hline 13 & 1,5 & 0,3 & 6,8 & 90,1 \\
\hline 14 & 1,4 & 0,3 & 6,3 & 91,3 \\
\hline 15 & 1,6 & 0,3 & 7,3 & 88,7 \\
\hline 16 & 1,5 & 0,3 & 7,1 & 89,1 \\
\hline 17 & 1,5 & 0,3 & 7,2 & 88,9 \\
\hline 18 & 1,5 & 0,3 & 7,3 & 88,8 \\
\hline
\end{tabular}


A Tabela 39 trás o cálculo da incerteza combinada para as medições feitas a $3000 \mathrm{rpm}$, com pressão de sistema de $15 \mathrm{kPa}$, utilizando a mistura de $30 \%$ etilenoglicol e $70 \%$ água como fluido.

Tabela A37- Incerteza combinada para 3000 rpm, mistura 30/70 com 15 kPa de pressão de sistema.

\begin{tabular}{|c|c|c|c|c|c|}
\hline Índice & $\mathbf{T}\left[{ }^{\circ} \mathbf{C}\right]$ & $\mathbf{P}$ 1 [kPa] & $\mathbf{P 2}[\mathbf{k P a}]$ & $\mathbf{D P}[\mathbf{k P a}]$ & $\begin{array}{c}\mathbf{u}_{\mathbf{c}} \\
{[\%]}\end{array}$ \\
\hline 1 & 85 & 14,1 & 56,8 & 42,7 & 2,4 \\
\hline 2 & 87 & 13,1 & 55,8 & 42,7 & 2,3 \\
\hline 3 & 89 & 11,6 & 54,3 & 42,7 & 2,3 \\
\hline 4 & 91 & 12,8 & 55,1 & 42,3 & 2,3 \\
\hline 5 & 93 & 12,5 & 55,0 & 42,5 & 2,3 \\
\hline 6 & 95 & 12,2 & 54,9 & 42,7 & 2,3 \\
\hline 7 & 97 & 11,2 & 53,6 & 42,4 & 2,2 \\
\hline 8 & 99 & 12,0 & 54,5 & 42,5 & 2,3 \\
\hline 9 & 101 & 13,8 & 56,4 & 42,6 & 2,4 \\
\hline 10 & 102 & 12,2 & 54,9 & 42,7 & 2,3 \\
\hline 11 & 103 & 10,6 & 53,1 & 42,5 & 2,2 \\
\hline 12 & 104 & 9,9 & 52,7 & 42,8 & 2,2 \\
\hline 13 & 105 & 10,1 & 52,4 & 42,3 & 2,2 \\
\hline 14 & 106 & 11,2 & 53,0 & 41,8 & 2,3 \\
\hline 15 & 107 & 11,8 & 52,9 & 41,1 & 2,3 \\
\hline 16 & 108 & 11,5 & 51,6 & 40,1 & 2,3 \\
\hline 17 & 109 & 11,3 & 50,7 & 39,4 & 2,3 \\
\hline 18 & 110 & 11,4 & 48,9 & 37,5 & 2,3 \\
\hline
\end{tabular}

As Tabelas A38 e A39 apresentam o cálculo dos coeficientes de sensibilidade das medições das pressões de entrada e saída, respectivamente. A primeira coluna referencia a seqüência de medição. A segunda, terceira e quarta coluna contém a informação para cada um dos componentes da Eq. 13. A quinta coluna traz a contribuição para a incerteza combinada. 
Tabela A38- Coeficientes de sensibilidade para a pressão de entrada a $3000 \mathrm{rpm}$, mistura 30/70 com $15 \mathrm{kPa}$ de pressão de sistema.

\begin{tabular}{|c|c|c|c|c|}
\hline Índice & $S_{P 1}$ & $S_{P 1 x} u_{p}$ & $100 \times\left(S_{P 1 \times} u_{p}\right)^{2}$ & $\begin{array}{l}\text { Contribuição do Transdutor } \\
\text { de Pressão da Entrada[\%] }\end{array}$ \\
\hline 1 & $-0,3$ & $-0,1$ & 0,3 & 5,8 \\
\hline 2 & $-0,3$ & $-0,1$ & 0,3 & 5,2 \\
\hline 3 & $-0,3$ & 0,0 & 0,2 & 4,4 \\
\hline 4 & $-0,3$ & $-0,1$ & 0,3 & 5,1 \\
\hline 5 & $-0,3$ & $-0,1$ & 0,3 & 4,9 \\
\hline 6 & $-0,3$ & 0,0 & 0,2 & 4,7 \\
\hline 7 & $-0,3$ & 0,0 & 0,2 & 4,2 \\
\hline 8 & $-0,3$ & 0,0 & 0,2 & 4,6 \\
\hline 9 & $-0,3$ & $-0,1$ & 0,3 & 5,7 \\
\hline 10 & $-0,3$ & 0,0 & 0,2 & 4,7 \\
\hline 11 & $-0,2$ & 0,0 & 0,2 & 3,8 \\
\hline 12 & $-0,2$ & 0,0 & 0,2 & 3,4 \\
\hline 13 & $-0,2$ & 0,0 & 0,2 & 3,6 \\
\hline 14 & $-0,3$ & 0,0 & 0,2 & 4,3 \\
\hline 15 & $-0,3$ & 0,0 & 0,2 & 4,7 \\
\hline 16 & $-0,3$ & 0,0 & 0,2 & 4,7 \\
\hline 17 & $-0,3$ & 0,0 & 0,2 & 4,7 \\
\hline 18 & $-0,3$ & $-0,1$ & 0,3 & 5,2 \\
\hline
\end{tabular}

Tabela A39 - Coeficientes de sensibilidade para a pressão de saída a $3000 \mathrm{rpm}$, mistura 30/70 com $15 \mathrm{kPa}$ de pressão de sistema.

\begin{tabular}{|c|c|c|c|c|}
\hline Índice & $\mathbf{S}_{\mathbf{P} \mathbf{2}}$ & $\mathbf{S}_{\mathbf{P} \mathbf{2} \mathbf{x}} \mathbf{u}_{\mathbf{p}}$ & $\mathbf{1 0 0} \mathbf{x}\left(\mathbf{S}_{\mathbf{P} \mathbf{2}} \mathbf{u}_{\mathbf{p}} \mathbf{~}^{\mathbf{2}}\right.$ & $\begin{array}{c}\text { Contribuição do Transdutor } \\
\mathbf{d e ~ P r e s s a ̃ o ~ d a ~ S a i ́ d a ~ [ \% ] ~}\end{array}$ \\
\hline 1 & 1,3 & 0,2 & 5,4 & 94,2 \\
\hline 2 & 1,3 & 0,2 & 5,2 & 94,8 \\
\hline 3 & 1,3 & 0,2 & 4,9 & 95,6 \\
\hline 4 & 1,3 & 0,2 & 5,1 & 94,9 \\
\hline 5 & 1,3 & 0,2 & 5,1 & 95,1 \\
\hline 6 & 1,3 & 0,2 & 5,0 & 95,3 \\
\hline 7 & 1,3 & 0,2 & 4,8 & 95,8 \\
\hline 8 & 1,3 & 0,2 & 5,0 & 95,4 \\
\hline 9 & 1,3 & 0,2 & 5,3 & 94,3 \\
\hline 10 & 1,3 & 0,2 & 5,0 & 95,3 \\
\hline 11 & 1,2 & 0,2 & 4,7 & 96,2 \\
\hline 12 & 1,2 & 0,2 & 4,6 & 96,6 \\
\hline 13 & 1,2 & 0,2 & 4,6 & 96,4 \\
\hline 14 & 1,3 & 0,2 & 4,9 & 95,7 \\
\hline 15 & 1,3 & 0,2 & 5,0 & 95,3 \\
\hline 16 & 1,3 & 0,2 & 5,0 & 95,3 \\
\hline 17 & 1,3 & 0,2 & 5,0 & 95,3 \\
\hline 18 & 1,3 & 0,2 & 5,1 & 94,8 \\
\hline
\end{tabular}


A Tabela A40 trás o cálculo da incerteza combinada para as medições feitas a $4000 \mathrm{rpm}$, com pressão de sistema de $15 \mathrm{kPa}$, utilizando a mistura de $30 \%$ etilenoglicol e $70 \%$ água como fluido.

Tabela A40 - Incerteza combinada para 4000 rpm, mistura 30/70 com 15 kPa de pressão de sistema.

\begin{tabular}{|c|c|c|c|c|c|}
\hline Índice & $\mathbf{T}\left[{ }^{\circ} \mathbf{C}\right]$ & $\mathbf{P 1}[\mathbf{k P a}]$ & $\mathbf{P} 2 \mathbf{[ k P a}]$ & $\mathbf{D P}[\mathbf{k P a}]$ & $\begin{array}{c}\mathbf{u}_{\mathbf{c}} \\
{[\%]}\end{array}$ \\
\hline 1 & 85 & 13,8 & 86,9 & 73,1 & 2,1 \\
\hline 2 & 87 & 13,6 & 86,6 & 73,0 & 2,1 \\
\hline 3 & 89 & 12,8 & 86,4 & 73,6 & 2,1 \\
\hline 4 & 91 & 12,4 & 85,7 & 73,3 & 2,1 \\
\hline 5 & 93 & 12,1 & 84,2 & 72,1 & 2,1 \\
\hline 6 & 95 & 12,5 & 85,1 & 72,6 & 2,1 \\
\hline 7 & 97 & 12,8 & 84,9 & 72,1 & 2,1 \\
\hline 8 & 99 & 11,8 & 84,5 & 72,7 & 2,0 \\
\hline 9 & 101 & 11,9 & 84,5 & 72,6 & 2,0 \\
\hline 10 & 102 & 12,1 & 82,6 & 70,5 & 2,1 \\
\hline 11 & 103 & 9,7 & 77,3 & 67,6 & 2,0 \\
\hline 12 & 104 & 10,5 & 76,7 & 66,2 & 2,0 \\
\hline 13 & 105 & 10,8 & 70,7 & 59,9 & 2,1 \\
\hline 14 & 106 & 11,2 & 67,4 & 56,2 & 2,1 \\
\hline 15 & 107 & 10,2 & 63,0 & 52,8 & 2,1 \\
\hline 16 & 108 & 11,8 & 62,9 & 51,1 & 2,2 \\
\hline 17 & 110 & 11,6 & 58,0 & 46,4 & 2,2 \\
\hline
\end{tabular}

As Tabelas A41 e A42 apresentam o cálculo dos coeficientes de sensibilidade das medições das pressões de entrada e saída, respectivamente. A primeira coluna referencia a seqüência de medição. A segunda, terceira e quarta coluna contém a informação para cada um dos componentes da Eq. 13. A quinta coluna traz a contribuição para a incerteza combinada. 
Tabela A41 - Coeficientes de sensibilidade para a pressão de entrada a $4000 \mathrm{rpm}$, mistura 30/70 com $15 \mathrm{kPa}$ de pressão de sistema.

\begin{tabular}{|c|c|c|c|c|}
\hline Índice & $\mathbf{S}_{\mathbf{p} \mathbf{1}}$ & $\mathbf{S}_{\mathbf{p} \mathbf{x} \mathbf{x}} \mathbf{u}_{\mathbf{p}}$ & $\mathbf{1 0 0} \mathbf{x}\left(\mathbf{S}_{\mathbf{p} \mathbf{x} \mathbf{x}} \mathbf{u}_{\mathbf{p}}\right)^{\mathbf{2}}$ & $\begin{array}{c}\text { Contribuição do Transdutor } \\
\text { de Pressão da Entrada[\%] }\end{array}$ \\
\hline 1 & $-0,2$ & 0,0 & 0,1 & 2,5 \\
\hline 2 & $-0,2$ & 0,0 & 0,1 & 2,4 \\
\hline 3 & $-0,2$ & 0,0 & 0,1 & 2,1 \\
\hline 4 & $-0,2$ & 0,0 & 0,1 & 2,1 \\
\hline 5 & $-0,2$ & 0,0 & 0,1 & 2,0 \\
\hline 6 & $-0,2$ & 0,0 & 0,1 & 2,1 \\
\hline 7 & $-0,2$ & 0,0 & 0,1 & 2,2 \\
\hline 8 & $-0,2$ & 0,0 & 0,1 & 1,9 \\
\hline 9 & $-0,2$ & 0,0 & 0,1 & 1,9 \\
\hline 10 & $-0,2$ & 0,0 & 0,1 & 2,1 \\
\hline 11 & $-0,1$ & 0,0 & 0,1 & 1,5 \\
\hline 12 & $-0,2$ & 0,0 & 0,1 & 2,3 \\
\hline 13 & $-0,2$ & 0,0 & 0,1 & 2,7 \\
\hline 14 & $-0,2$ & 0,0 & 0,1 & 2,6 \\
\hline 15 & $-0,2$ & 0,0 & 0,1 & 3,4 \\
\hline 16 & $-0,2$ & 0,0 & 0,2 & 3,8 \\
\hline 17 & $-0,3$ & 0,0 & 0,2 & \\
\hline
\end{tabular}

Tabela A42 - Coeficientes de sensibilidade para a pressão de saída a $4000 \mathrm{rpm}$, mistura 30/70 com $15 \mathrm{kPa}$ de pressão de sistema.

\begin{tabular}{|c|c|c|c|c|}
\hline Índice & $\mathbf{S}_{\mathbf{P} \mathbf{2}}$ & $\mathbf{S}_{\mathbf{P} \mathbf{2} \mathbf{}} \mathbf{u}_{\mathbf{p}}$ & $\mathbf{1 0 0} \mathbf{x}\left(\mathbf{S}_{\mathbf{P} \mathbf{x}} \mathbf{\mathbf { u } _ { \mathbf { p } }} \mathbf{2}^{\mathbf{2}}\right.$ & $\begin{array}{c}\text { Contribuição do Transdutor } \\
\mathbf{d e ~ P r e s s a ̃ o ~ d a ~ S a i ́ d a ~ [ \% ] ~}\end{array}$ \\
\hline 1 & 1,2 & 0,2 & 4,3 & 97,5 \\
\hline 2 & 1,2 & 0,2 & 4,3 & 97,6 \\
\hline 3 & 1,2 & 0,2 & 4,2 & 97,9 \\
\hline 4 & 1,2 & 0,2 & 4,1 & 97,9 \\
\hline 5 & 1,2 & 0,2 & 4,1 & 98,0 \\
\hline 6 & 1,2 & 0,2 & 4,2 & 97,9 \\
\hline 7 & 1,2 & 0,2 & 4,2 & 97,8 \\
\hline 8 & 1,2 & 0,2 & 4,1 & 98,1 \\
\hline 9 & 1,2 & 0,2 & 4,1 & 98,1 \\
\hline 10 & 1,2 & 0,2 & 4,2 & 97,9 \\
\hline 11 & 1,1 & 0,2 & 4,0 & 98,5 \\
\hline 12 & 1,2 & 0,2 & 4,1 & 98,2 \\
\hline 13 & 1,2 & 0,2 & 4,2 & 97,7 \\
\hline 14 & 1,2 & 0,2 & 4,4 & 97,3 \\
\hline 15 & 1,2 & 0,2 & 4,3 & 97,4 \\
\hline 16 & 1,2 & 0,2 & 4,6 & 96,6 \\
\hline 17 & 1,3 & 0,2 & 4,7 & 96,2 \\
\hline
\end{tabular}


A Tabela A43 trás o cálculo da incerteza combinada para as medições feitas a $5000 \mathrm{rpm}$, com pressão de sistema de $15 \mathrm{kPa}$, utilizando a mistura de $30 \%$ etilenoglicol e $70 \%$ água como fluido.

Tabela A43 - Incerteza combinada para 5000 rpm, mistura 30/70 com 15 kPa de pressão de sistema.

\begin{tabular}{|c|c|c|c|c|c|}
\hline Índice & $\mathbf{T}\left[{ }^{\circ} \mathbf{C}\right]$ & $\mathbf{P}$ 1 [kPa] & $\mathbf{P 2}[\mathbf{k P a}]$ & $\mathbf{D P}[\mathbf{k P a}]$ & $\begin{array}{c}\mathbf{u}_{\mathbf{c}} \\
{[\%]}\end{array}$ \\
\hline 1 & 85 & 10,9 & 109,3 & 98,4 & 1,9 \\
\hline 2 & 87 & 10,8 & 109,9 & 99,1 & 1,9 \\
\hline 3 & 90 & 11,3 & 110,2 & 98,9 & 1,9 \\
\hline 4 & 93 & 11,4 & 110,0 & 98,6 & 2,0 \\
\hline 5 & 95 & 11,2 & 109,4 & 98,2 & 1,9 \\
\hline 6 & 97 & 11,2 & 109,5 & 98,3 & 1,9 \\
\hline 7 & 98 & 10,3 & 105,2 & 94,9 & 1,9 \\
\hline 8 & 99 & 11,7 & 101,0 & 89,3 & 2,0 \\
\hline 9 & 100 & 11,0 & 91,7 & 80,7 & 2,0 \\
\hline 10 & 101 & 11,2 & 88,5 & 77,3 & 2,0 \\
\hline 11 & 102 & 10,7 & 73,8 & 63,1 & 2,1 \\
\hline 12 & 103 & 10,5 & 71,5 & 61,0 & 2,1 \\
\hline 13 & 104 & 10,8 & 68,7 & 57,9 & 2,1 \\
\hline 14 & 105 & 10,2 & 65,7 & 55,5 & 2,1 \\
\hline 15 & 107 & 10,4 & 63,1 & 52,7 & 2,1 \\
\hline 16 & 110 & 10,8 & 60,2 & 49,4 & 2,2 \\
\hline
\end{tabular}

As Tabelas A44 e A45 apresentam o cálculo dos coeficientes de sensibilidade das medições das pressões de entrada e saída, respectivamente. A primeira coluna referencia a seqüência de medição. A segunda, terceira e quarta coluna contém a informação para cada um dos componentes da Eq. 13. A quinta coluna traz a contribuição para a incerteza combinada. 
Tabela A44 - Coeficientes de sensibilidade para a pressão de entrada a 5000 rpm, mistura 30/70 com 15 kPa de pressão de sistema.

\begin{tabular}{|c|c|c|c|c|}
\hline Índice & $\mathbf{S}_{\mathbf{P} \mathbf{1}}$ & $\mathbf{S}_{\mathbf{P} \mathbf{1} \mathbf{x}} \mathbf{u}_{\mathbf{p}}$ & $\mathbf{1 0 0} \mathbf{x}\left(\mathbf{S}_{\mathbf{P} \mathbf{x} \mathbf{~} \mathbf{u}_{\mathbf{p}} \mathbf{~}^{\mathbf{2}}}\right.$ & $\begin{array}{c}\text { Contribuição do Transdutor } \\
\text { de Pressão da Entrada[\%] }\end{array}$ \\
\hline 1 & $-0,1$ & 0,0 & 0,0 & 1,0 \\
\hline 2 & $-0,1$ & 0,0 & 0,0 & 1,0 \\
\hline 3 & $-0,1$ & 0,0 & 0,0 & 1,0 \\
\hline 4 & $-0,1$ & 0,0 & 0,0 & 1,1 \\
\hline 5 & $-0,1$ & 0,0 & 0,0 & 1,0 \\
\hline 6 & $-0,1$ & 0,0 & 0,0 & 1,0 \\
\hline 7 & $-0,1$ & 0,0 & 0,0 & 1,0 \\
\hline 8 & $-0,1$ & 0,0 & 0,1 & 1,3 \\
\hline 9 & $-0,1$ & 0,0 & 0,1 & 1,4 \\
\hline 10 & $-0,1$ & 0,0 & 0,1 & 1,6 \\
\hline 11 & $-0,2$ & 0,0 & 0,1 & 2,1 \\
\hline 12 & $-0,2$ & 0,0 & 0,1 & 2,1 \\
\hline 13 & $-0,2$ & 0,0 & 0,1 & 2,4 \\
\hline 14 & $-0,2$ & 0,0 & 0,1 & 2,4 \\
\hline 15 & $-0,2$ & 0,0 & 0,1 & 3,1 \\
\hline 16 & $-0,2$ & 0,0 & 0,1 & \\
\hline
\end{tabular}

Tabela A45 - Coeficientes de sensibilidade para a pressão de saída a 5000 rpm, mistura 30/70 com 15 kPa de pressão de sistema.

\begin{tabular}{|c|c|c|c|c|}
\hline Índice & $S_{P 2}$ & $S_{P 2 \times} u_{p}$ & $100 \times\left(S_{P 2} \times u_{p}\right)^{2}$ & $\begin{array}{l}\text { Contribuição do Transdutor } \\
\text { de Pressão da Saída [\%] }\end{array}$ \\
\hline 1 & 1,1 & 0,2 & 3,7 & 99,0 \\
\hline 2 & 1,1 & 0,2 & 3,7 & 99,0 \\
\hline 3 & 1,1 & 0,2 & 3,8 & 99,0 \\
\hline 4 & 1,1 & 0,2 & 3,8 & 98,9 \\
\hline 5 & 1,1 & 0,2 & 3,8 & 99,0 \\
\hline 6 & 1,1 & 0,2 & 3,8 & 99,0 \\
\hline 7 & 1,1 & 0,2 & 3,7 & 99,0 \\
\hline 8 & 1,1 & 0,2 & 3,9 & 98,7 \\
\hline 9 & 1,1 & 0,2 & 3,9 & 98,6 \\
\hline 10 & 1,1 & 0,2 & 4,0 & 98,4 \\
\hline 11 & 1,2 & 0,2 & 4,1 & 97,9 \\
\hline 12 & 1,2 & 0,2 & 4,2 & 97,9 \\
\hline 13 & 1,2 & 0,2 & 4,3 & 97,6 \\
\hline 14 & 1,2 & 0,2 & 4,2 & 97,6 \\
\hline 15 & 1,2 & 0,2 & 4,3 & 97,4 \\
\hline 16 & 1,2 & 0,2 & 4,5 & 96,9 \\
\hline
\end{tabular}


A Tabela A46 trás o cálculo da incerteza combinada para as medições feitas a $1000 \mathrm{rpm}$, com pressão de sistema de $50 \mathrm{kPa}$, utilizando a mistura de $30 \%$ etilenoglicol e $70 \%$ água como fluido.

Tabela A46 - Incerteza combinada para 1000 rpm, mistura 30/70 com 50 kPa de pressão de sistema.

\begin{tabular}{|c|c|c|c|c|c|}
\hline Índice & $\mathbf{T}\left[{ }^{\mathbf{C}} \mathbf{C}\right]$ & $\mathbf{P 1}[\mathbf{k P a}]$ & $\mathbf{P} 2[\mathbf{k P a}]$ & $\begin{array}{c}\mathbf{D P} \\
{[\mathbf{k P a}]}\end{array}$ & $\begin{array}{c}\mathbf{u}_{\mathbf{c}} \\
{[\%]}\end{array}$ \\
\hline 1 & 85 & 42,9 & 50,2 & 7,3 & 15,7 \\
\hline 2 & 92 & 43,1 & 50,3 & 7,2 & 16,0 \\
\hline 3 & 97 & 43,5 & 50,9 & 7,4 & 15,7 \\
\hline 4 & 100 & 43,2 & 50,5 & 7,3 & 15,8 \\
\hline 5 & 101 & 43,4 & 50,6 & 7,2 & 16,1 \\
\hline 6 & 102 & 43,4 & 50,7 & 7,3 & 16,0 \\
\hline 7 & 103 & 44,1 & 51,2 & 7,1 & 16,5 \\
\hline 8 & 105 & 42,8 & 50,1 & 7,3 & 15,7 \\
\hline 9 & 107 & 42,5 & 49,9 & 7,4 & 15,4 \\
\hline 10 & 110 & 42,2 & 49,6 & 7,4 & 15,3 \\
\hline
\end{tabular}

As Tabelas A46 e A47 apresentam o cálculo dos coeficientes de sensibilidade das medições das pressões de entrada e saída, respectivamente. A primeira coluna referencia a seqüência de medição. A segunda, terceira e quarta coluna contém a informação para cada um dos componentes da Eq. 13. A quinta coluna traz a contribuição para a incerteza combinada.

Tabela A46 - Coeficientes de sensibilidade para a pressão de entrada a 1000 rpm, mistura 30/70 com 50 kPa de pressão de sistema.

\begin{tabular}{|c|c|c|c|c|}
\hline Índice & $S_{\mathrm{P} 1}$ & $S_{P 1 \times} u_{p}$ & $100 \times\left(S_{p 1} \times u_{p}\right)^{2}$ & $\begin{array}{l}\text { Contribuição do } \\
\text { Transdutor de Pressão da } \\
\text { Entrada[\%] }\end{array}$ \\
\hline 1 & $\begin{array}{l}-5,9 \\
\end{array}$ & $\begin{array}{l}-1,0 \\
\end{array}$ & 104,6 & 42,2 \\
\hline 2 & $-6,0$ & $\begin{array}{l}-1,0 \\
\end{array}$ & 108,5 & 42,3 \\
\hline 3 & $\begin{array}{l}-5,9 \\
\end{array}$ & $\begin{array}{l}-1,0 \\
\end{array}$ & 104,6 & 42,2 \\
\hline 4 & $-5,9$ & $-1,0$ & 106,0 & 42,3 \\
\hline 5 & $-6,0$ & $-1,0$ & 110,0 & 42,4 \\
\hline 6 & $-6,0$ & $-1,0$ & 108,5 & 42,3 \\
\hline 7 & $-6,2$ & $-1,1$ & 116,0 & 42,6 \\
\hline 8 & $-5,9$ & $-1,0$ & 104,1 & 42,2 \\
\hline 9 & $-5,7$ & $\begin{array}{l}-1,0 \\
\end{array}$ & 99,9 & 42,0 \\
\hline 10 & $-5,7$ & $-1,0$ & 98,5 & 42,0 \\
\hline
\end{tabular}


Tabela A47 - Coeficientes de sensibilidade para a pressão de saída a 1000 rpm, mistura 30/70 com 50 kPa de pressão de sistema.

\begin{tabular}{|c|c|c|c|c|}
\hline Índice & $\mathbf{S}_{\mathbf{P} \mathbf{2}}$ & $\mathbf{S}_{\mathbf{P} \mathbf{2}} \mathbf{u}_{\mathbf{p}}$ & $\mathbf{1 0 0} \mathbf{x}\left(\mathbf{S}_{\mathbf{P} \mathbf{2}} \mathbf{\mathbf { u } _ { \mathbf { p } }}\right)^{\mathbf{2}}$ & $\begin{array}{c}\text { Contribuição do Transdutor } \\
\text { de Pressão da Saída [\%] }\end{array}$ \\
\hline 1 & 6,9 & 1,2 & 143,2 & 57,8 \\
\hline 2 & 7,0 & 1,2 & 147,8 & 57,7 \\
\hline 3 & 6,9 & 1,2 & 143,2 & 57,8 \\
\hline 4 & 6,9 & 1,2 & 144,9 & 57,7 \\
\hline 5 & 7,0 & 1,2 & 149,5 & 57,6 \\
\hline 6 & 7,0 & 1,2 & 147,8 & 57,7 \\
\hline 7 & 7,2 & 1,3 & 156,5 & 57,4 \\
\hline 8 & 6,9 & 1,2 & 142,6 & 57,8 \\
\hline 9 & 6,7 & 1,2 & 137,7 & 58,0 \\
\hline 10 & 6,7 & 1,2 & 136,0 & 58,0 \\
\hline
\end{tabular}

A Tabela A48 trás o cálculo da incerteza combinada para as medições feitas a 2000 rpm, com pressão de sistema de $50 \mathrm{kPa}$, utilizando a mistura de 30\% etilenoglicol e $70 \%$ água como fluido.

Tabela A48 - Incerteza combinada para 2000 rpm, mistura 30/70 com 50 kPa de pressão de sistema.

\begin{tabular}{|c|c|c|c|c|c|}
\hline Índice & $\mathbf{T}\left[{ }^{\circ} \mathbf{C}\right]$ & $\mathbf{P}$ [ $[\mathbf{k P a}]$ & $\mathbf{P 2}[\mathbf{k P a}]$ & $\mathbf{D P}[\mathbf{k P a}]$ & $\begin{array}{c}\mathbf{u}_{\mathbf{c}} \\
{[\%]}\end{array}$ \\
\hline 1 & 85 & 44,5 & 65,1 & 20,6 & 6,7 \\
\hline 2 & 92 & 44,3 & 65,2 & 20,9 & 6,6 \\
\hline 3 & 97 & 44,4 & 64,8 & 20,4 & 6,7 \\
\hline 4 & 100 & 44,1 & 64,9 & 20,8 & 6,6 \\
\hline 5 & 101 & 44,3 & 65,0 & 20,7 & 6,6 \\
\hline 6 & 102 & 44,8 & 65,5 & 20,7 & 6,7 \\
\hline 7 & 103 & 44,7 & 65,5 & 20,8 & 6,6 \\
\hline 8 & 105 & 44,8 & 65,4 & 20,6 & 6,7 \\
\hline 9 & 107 & 44,5 & 65,3 & 20,8 & 6,6 \\
\hline 10 & 109 & 44,6 & 65,3 & 20,7 & 6,7 \\
\hline 11 & 110 & 43,8 & 64,7 & 20,9 & 6,5 \\
\hline
\end{tabular}

As Tabelas A49 e A50 apresentam o cálculo dos coeficientes de sensibilidade das medições das pressões de entrada e saída, respectivamente. A primeira coluna referencia a seqüência de medição. $A$ segunda, terceira e quarta coluna contém a informação para cada um dos 
componentes da Eq. 13. A quinta coluna traz a contribuição para a incerteza combinada.

Tabela A49 - Coeficientes de sensibilidade para a pressão de entrada a 2000 rpm, mistura 30/70 com $50 \mathrm{kPa}$ de pressão de sistema.

\begin{tabular}{|c|c|c|c|c|}
\hline Índice & $S_{P_{1}}$ & $S_{p_{1} \times} u_{p}$ & $100 \times\left(S_{P_{1} \times} u_{p}\right)^{2}$ & $\begin{array}{l}\text { Contribuição do Transdutor } \\
\text { de Pressão da Entrada[\%] }\end{array}$ \\
\hline 1 & $-2,2$ & $-0,4$ & 14,1 & 31,8 \\
\hline 2 & $-2,1$ & $-0,4$ & 13,6 & 31,6 \\
\hline 3 & $-2,2$ & $-0,4$ & 14,3 & 31,9 \\
\hline 4 & $-2,1$ & $-0,4$ & 13,6 & 31,6 \\
\hline 5 & $-2,1$ & $-0,4$ & 13,9 & 31,7 \\
\hline 6 & $-2,2$ & $-0,4$ & 14,2 & 31,9 \\
\hline 7 & $-2,2$ & $-0,4$ & 14,0 & 31,8 \\
\hline 8 & $-2,2$ & $-0,4$ & 14,3 & 31,9 \\
\hline 9 & $-2,1$ & $-0,4$ & 13,9 & 31,7 \\
\hline 10 & $-2,2$ & $-0,4$ & 14,1 & 31,8 \\
\hline 11 & $-2,1$ & $-0,4$ & 13,4 & 31,5 \\
\hline
\end{tabular}

Tabela A50 - Coeficientes de sensibilidade para a pressão de saída a 2000 rpm, mistura 30/70 com $50 \mathrm{kPa}$ de pressão de sistema.

\begin{tabular}{|c|c|c|c|c|}
\hline Índice & $\mathbf{S}_{\mathbf{P} 2}$ & $\mathbf{S}_{\mathbf{P} \mathbf{2}} \mathbf{u}_{\mathbf{p}}$ & $\mathbf{1 0 0} \mathbf{x}\left(\mathbf{S}_{\mathbf{P} \mathbf{2}} \mathbf{\mathbf { u } _ { \mathbf { p } }} \mathbf{\mathbf { p } ^ { 2 }}\right.$ & $\begin{array}{c}\text { Contribuição do Transdutor } \\
\mathbf{d e} \text { Pressão da Saída [\%] }\end{array}$ \\
\hline 1 & 3,2 & 0,5 & 30,2 & 68,2 \\
\hline 2 & 3,1 & 0,5 & 29,5 & 68,4 \\
\hline 3 & 3,2 & 0,6 & 30,5 & 68,1 \\
\hline 4 & 3,1 & 0,5 & 29,4 & 68,4 \\
\hline 5 & 3,1 & 0,5 & 29,9 & 68,3 \\
\hline 6 & 3,2 & 0,6 & 30,4 & 68,1 \\
\hline 7 & 3,2 & 0,5 & 30,1 & 68,2 \\
\hline 8 & 3,2 & 0,6 & 30,5 & 68,1 \\
\hline 9 & 3,1 & 0,5 & 29,9 & 68,3 \\
\hline 10 & 3,2 & 0,5 & 30,2 & 68,2 \\
\hline 11 & 3,1 & 0,5 & 29,1 & 68,5 \\
\hline
\end{tabular}

A Tabela A51 trás o cálculo da incerteza combinada para as medições feitas a $3000 \mathrm{rpm}$, com pressão de sistema de $50 \mathrm{kPa}$, utilizando a mistura de $30 \%$ etilenoglicol e $70 \%$ água como fluido. 
Tabela A51 - Incerteza combinada para 3000 rpm, mistura 30/70 com 50 $\mathrm{kPa}$ de pressão de sistema.

\begin{tabular}{|c|c|c|c|c|c|}
\hline Índice & $\mathbf{T}\left[{ }^{\circ} \mathbf{C}\right]$ & $\mathbf{P}$ [ $[\mathbf{k P a}]$ & $\mathbf{P} 2[\mathbf{k P a}]$ & $\mathbf{D P}[\mathbf{k P a}]$ & $\begin{array}{c}\mathbf{u}_{\mathbf{c}} \\
{[\%]}\end{array}$ \\
\hline 1 & 85 & 46,4 & 89,8 & 43,4 & 4,1 \\
\hline 2 & 92 & 46,7 & 89,8 & 43,1 & 4,1 \\
\hline 3 & 97 & 46,6 & 89,9 & 43,3 & 4,1 \\
\hline 4 & 100 & 46,8 & 89,7 & 42,9 & 4,1 \\
\hline 5 & 101 & 46,5 & 89,6 & 43,1 & 4,1 \\
\hline 6 & 102 & 47,0 & 90,6 & 43,6 & 4,1 \\
\hline 7 & 103 & 46,8 & 90,1 & 43,3 & 4,1 \\
\hline 8 & 105 & 46,7 & 89,7 & 43,0 & 4,1 \\
\hline 9 & 107 & 46,4 & 89,5 & 43,1 & 4,1 \\
\hline 10 & 109 & 46,1 & 89,2 & 43,1 & 4,1 \\
\hline 11 & 110 & 45,9 & 88,9 & 43,0 & 4,0 \\
\hline
\end{tabular}

As Tabelas A52 e A53 apresentam o cálculo dos coeficientes de sensibilidade das medições das pressões de entrada e saída, respectivamente. A primeira coluna referencia a seqüência de medição. A segunda, terceira e quarta coluna contém a informação para cada um dos componentes da Eq. 13. A quinta coluna traz a contribuição para a incerteza combinada.

Tabela A52 - Coeficientes de sensibilidade para a pressão de entrada a $3000 \mathrm{rpm}$, mistura $30 / 70 \mathrm{com} 50 \mathrm{kPa}$ de pressão de sistema.

\begin{tabular}{|c|c|c|c|c|}
\hline Índice & $\mathbf{S}_{\mathbf{P} \mathbf{1}}$ & $\mathbf{S}_{\mathbf{P} \mathbf{1} \mathbf{x}} \mathbf{u}_{\mathbf{p}}$ & $\mathbf{1 0 0} \mathbf{x}\left(\mathbf{S}_{\mathbf{P} \mathbf{x} \mathbf{x}} \mathbf{u}_{\mathbf{p}}\right)^{\mathbf{2}}$ & $\begin{array}{c}\text { Contribuição do Transdutor } \\
\text { de Pressão da Entrada[\%] }\end{array}$ \\
\hline 1 & $-1,1$ & $-0,2$ & 3,5 & 21,1 \\
\hline 2 & $-1,1$ & $-0,2$ & 3,6 & 21,3 \\
\hline 3 & $-1,1$ & $-0,2$ & 3,5 & 21,2 \\
\hline 4 & $-1,1$ & $-0,2$ & 3,6 & 21,4 \\
\hline 5 & $-1,1$ & $-0,2$ & 3,5 & 21,2 \\
\hline 6 & $-1,1$ & $-0,2$ & 3,5 & 21,2 \\
\hline 7 & $-1,1$ & $-0,2$ & 3,5 & 21,3 \\
\hline 8 & $-1,1$ & $-0,2$ & 3,6 & 21,2 \\
\hline 9 & $-1,1$ & $-0,2$ & 3,5 & 21,1 \\
\hline 10 & $-1,1$ & $-0,2$ & 3,5 & 21,1 \\
\hline 11 & $-1,1$ & $-0,2$ & 3,5 & \\
\hline
\end{tabular}


Tabela A53 - Coeficientes de sensibilidade para a pressão de saída a 3000 rpm, mistura $30 / 70$ com 50 kPa de pressão de sistema.

\begin{tabular}{|c|c|c|c|c|}
\hline Índice & $S_{P 2}$ & $S_{P 2 \times} u_{p}$ & $100 \times\left(S_{P 2} \times u_{p}\right)^{2}$ & $\begin{array}{l}\text { Contribuição do Transdutor } \\
\text { de Pressão da Saída [\%] }\end{array}$ \\
\hline 1 & 2,1 & 0,4 & 13,0 & 78,9 \\
\hline 2 & 2,1 & 0,4 & 13,1 & 78,7 \\
\hline 3 & 2,1 & 0,4 & 13,1 & 78,8 \\
\hline 4 & 2,1 & 0,4 & 13,2 & 78,6 \\
\hline 5 & 2,1 & 0,4 & 13,1 & $\begin{array}{l}78,8 \\
\end{array}$ \\
\hline 6 & 2,1 & 0,4 & 13,1 & 78,8 \\
\hline 7 & 2,1 & 0,4 & 13,1 & 78,7 \\
\hline 8 & 2,1 & 0,4 & 13,2 & 78,7 \\
\hline 9 & 2,1 & 0,4 & 13,1 & 78,8 \\
\hline 10 & 2,1 & 0,4 & 13,0 & 78,9 \\
\hline 11 & 2,1 & 0,4 & 12,9 & 78,9 \\
\hline
\end{tabular}

A Tabela A54 trás o cálculo da incerteza combinada para as medições feitas a 4000 rpm, com pressão de sistema de $50 \mathrm{kPa}$, utilizando a mistura de $30 \%$ etilenoglicol e $70 \%$ água como fluido.

Tabela A54 - Incerteza combinada para 4000 rpm, mistura 30/70 com 50 kPa de pressão de sistema.

\begin{tabular}{|c|c|c|c|c|c|}
\hline Índice & $\mathbf{T}\left[{ }^{\circ} \mathbf{C}\right]$ & $\mathbf{P} 1[\mathbf{k P a}]$ & $\mathbf{P} 2[\mathbf{k P a}]$ & $\mathbf{D P}[\mathbf{k P a}]$ & $\begin{array}{c}\mathbf{u}_{\mathbf{c}} \\
{[\%]}\end{array}$ \\
\hline 1 & 85 & 45,9 & 120,1 & 74,2 & 3,0 \\
\hline 2 & 92 & 46,3 & 120,6 & 74,3 & 3,0 \\
\hline 3 & 97 & 46,1 & 120,2 & 74,1 & 3,0 \\
\hline 4 & 100 & 46,2 & 120,4 & 74,2 & 3,0 \\
\hline 5 & 101 & 46,0 & 120,5 & 74,5 & 3,0 \\
\hline 6 & 102 & 46,2 & 120,6 & 74,4 & 3,0 \\
\hline 7 & 103 & 46,1 & 120,5 & 74,4 & 3,0 \\
\hline 8 & 104 & 46,3 & 120,6 & 74,3 & 3,0 \\
\hline 9 & 105 & 46,6 & 119,7 & 73,1 & 3,1 \\
\hline 10 & 106 & 45,4 & 116,7 & 71,3 & 3,1 \\
\hline 11 & 107 & 44,8 & 115,6 & 70,8 & 3,0 \\
\hline 12 & 108 & 45,4 & 112,1 & 66,7 & 3,2 \\
\hline 13 & 109 & 46,1 & 111,8 & 65,7 & 3,2 \\
\hline 14 & 110 & 46,2 & 110,5 & 64,3 & 3,2 \\
\hline
\end{tabular}


As Tabelas A55 e A56 apresentam o cálculo dos coeficientes de sensibilidade das medições das pressões de entrada e saída, respectivamente. A primeira coluna referencia a seqüência de medição. A segunda, terceira e quarta coluna contém a informação para cada um dos componentes da Eq. 13. A quinta coluna traz a contribuição para a incerteza combinada.

Tabela A55 - Coeficientes de sensibilidade para a pressão de entrada a 4000 rpm, mistura 30/70 com 50 kPa de pressão de sistema.

\begin{tabular}{|c|c|c|c|c|}
\hline Índice & $\mathbf{S}_{\mathbf{P} 1}$ & $\mathbf{S}_{\mathbf{P}_{1} \mathbf{x}} \mathbf{u}_{\mathbf{p}}$ & $\mathbf{1 0 0} \mathbf{x}\left(\mathbf{S}_{\mathbf{P} \mathbf{1} \mathbf{x}} \mathbf{u}_{\mathbf{p}}\right)^{\mathbf{2}}$ & $\begin{array}{c}\text { Contribuição do Transdutor } \\
\text { de Pressão da Entrada[\%] }\end{array}$ \\
\hline 1 & $-0,6$ & $-0,1$ & 1,2 & 12,7 \\
\hline 2 & $-0,6$ & $-0,1$ & 1,2 & 12,8 \\
\hline 3 & $-0,6$ & $-0,1$ & 1,2 & 12,8 \\
\hline 4 & $-0,6$ & $-0,1$ & 1,2 & 12,8 \\
\hline 5 & $-0,6$ & $-0,1$ & 1,2 & 12,7 \\
\hline 6 & $-0,6$ & $-0,1$ & 1,2 & 12,8 \\
\hline 7 & $-0,6$ & $-0,1$ & 1,2 & 12,8 \\
\hline 8 & $-0,6$ & $-0,1$ & 1,2 & 12,8 \\
\hline 9 & $-0,6$ & $-0,1$ & 1,2 & 13,2 \\
\hline 10 & $-0,6$ & $-0,1$ & 1,2 & 13,1 \\
\hline 11 & $-0,6$ & $-0,1$ & 1,2 & 14,1 \\
\hline 12 & $-0,7$ & $-0,1$ & 1,4 & 14,5 \\
\hline 13 & $-0,7$ & $-0,1$ & 1,5 & 14,9 \\
\hline 14 & $-0,7$ & $-0,1$ & 1,6 & \\
\hline
\end{tabular}


Tabela A56 - Coeficientes de sensibilidade para a pressão de saída a 4000 rpm, mistura 30/70 com 50 kPa de pressão de sistema.

\begin{tabular}{|c|c|c|c|c|}
\hline Índice & $\mathbf{S}_{\mathbf{P} \mathbf{2}}$ & $\mathbf{S}_{\mathbf{P} \mathbf{2} \mathbf{x}} \mathbf{u}_{\mathbf{p}}$ & $\mathbf{1 0 0} \mathbf{x}\left(\mathbf{S}_{\mathbf{p} \mathbf{x}} \mathbf{\mathbf { u } _ { \mathbf { p } }} \mathbf{)}\right.$ & $\begin{array}{c}\text { Contribuição do Transdutor } \\
\mathbf{d e} \text { Pressão da Saída [\%] }\end{array}$ \\
\hline 1 & 1,6 & 0,3 & 7,9 & 87,3 \\
\hline 2 & 1,6 & 0,3 & 8,0 & 87,2 \\
\hline 3 & 1,6 & 0,3 & 8,0 & 87,2 \\
\hline 4 & 1,6 & 0,3 & 8,0 & 87,2 \\
\hline 5 & 1,6 & 0,3 & 7,9 & 87,3 \\
\hline 6 & 1,6 & 0,3 & 8,0 & 87,2 \\
\hline 7 & 1,6 & 0,3 & 7,9 & 87,2 \\
\hline 8 & 1,6 & 0,3 & 8,0 & 87,2 \\
\hline 9 & 1,6 & 0,3 & 8,1 & 86,8 \\
\hline 10 & 1,6 & 0,3 & 8,1 & 86,9 \\
\hline 11 & 1,6 & 0,3 & 8,1 & 86,9 \\
\hline 12 & 1,7 & 0,3 & 8,6 & 85,9 \\
\hline 13 & 1,7 & 0,3 & 8,8 & 85,5 \\
\hline 14 & 1,7 & 0,3 & 8,9 & 85,1 \\
\hline
\end{tabular}

A Tabela A57 trás o cálculo da incerteza combinada para as medições feitas a 5000 rpm, com pressão de sistema de $50 \mathrm{kPa}$, utilizando a mistura de $30 \%$ etilenoglicol e $70 \%$ água como fluido.

Tabela A57 - Incerteza combinada para 5000 rpm, mistura 30/70 com 50 kPa de pressão de sistema.

\begin{tabular}{|c|c|c|c|c|c|}
\hline Índice & $\mathbf{T}\left[{ }^{\circ} \mathbf{C}\right]$ & $\mathbf{P 1}[\mathbf{k P a}]$ & $\mathbf{P} 2[\mathbf{k P a}]$ & $\mathbf{D P}[\mathbf{k P a}]$ & $\begin{array}{c}\mathbf{u}_{\mathbf{c}} \\
{[\%]}\end{array}$ \\
\hline 1 & 85 & 41,2 & 141,4 & 100,2 & 2,6 \\
\hline 2 & 92 & 41,5 & 142,0 & 100,5 & 2,6 \\
\hline 3 & 97 & 41,3 & 141,6 & 100,3 & 2,6 \\
\hline 4 & 100 & 41,1 & 141,5 & 100,4 & 2,6 \\
\hline 5 & 101 & 41,8 & 141,9 & 100,1 & 2,6 \\
\hline 6 & 102 & 41,6 & 141,3 & 99,7 & 2,6 \\
\hline 7 & 103 & 41,2 & 138,7 & 97,5 & 2,6 \\
\hline 8 & 104 & 41,2 & 137,5 & 96,3 & 2,6 \\
\hline 9 & 105 & 41,6 & 137,5 & 95,9 & 2,6 \\
\hline 10 & 106 & 41,0 & 133,7 & 92,7 & 2,6 \\
\hline 11 & 107 & 41,6 & 131,5 & 89,9 & 2,7 \\
\hline 12 & 108 & 41,2 & 130,4 & 89,2 & 2,7 \\
\hline 13 & 109 & 41,8 & 126,8 & 85,0 & 2,7 \\
\hline 14 & 110 & 41,6 & 119,0 & 77,4 & 2,8 \\
\hline
\end{tabular}


As Tabelas A58 e A59 apresentam o cálculo dos coeficientes de sensibilidade das medições das pressões de entrada e saída, respectivamente. A primeira coluna referencia a seqüência de medição. A segunda, terceira e quarta coluna contém a informação para cada um dos componentes da Eq. 13. A quinta coluna traz a contribuição para a incerteza combinada.

Tabela A8 - Coeficientes de sensibilidade para a pressão de entrada a $5000 \mathrm{rpm}$, mistura $30 / 70 \mathrm{com} 50 \mathrm{kPa}$ de pressão de sistema.

\begin{tabular}{|c|c|c|c|c|}
\hline Índice & $\mathbf{S}_{\mathbf{P} \mathbf{1}}$ & $\mathbf{S}_{\mathbf{P} \mathbf{1} \mathbf{x}} \mathbf{u}_{\mathbf{p}}$ & $\mathbf{1 0 0} \mathbf{x}\left(\mathbf{S}_{\mathbf{p} \mathbf{x}} \mathbf{\mathbf { u } _ { \mathbf { p } }}\right)^{\mathbf{2}}$ & $\begin{array}{c}\text { Contribuição do Transdutor } \\
\text { de Pressão da Entrada[\%] }\end{array}$ \\
\hline 1 & $-0,4$ & $-0,1$ & 0,5 & 7,8 \\
\hline 2 & $-0,4$ & $-0,1$ & 0,5 & 7,9 \\
\hline 3 & $-0,4$ & $-0,1$ & 0,5 & 7,8 \\
\hline 4 & $-0,4$ & $-0,1$ & 0,5 & 7,8 \\
\hline 5 & $-0,4$ & $-0,1$ & 0,5 & 8,0 \\
\hline 6 & $-0,4$ & $-0,1$ & 0,5 & 8,0 \\
\hline 7 & $-0,4$ & $-0,1$ & 0,5 & 8,1 \\
\hline 8 & $-0,4$ & $-0,1$ & 0,6 & 8,2 \\
\hline 9 & $-0,4$ & $-0,1$ & 0,6 & 8,4 \\
\hline 10 & $-0,4$ & $-0,1$ & 0,6 & 8,6 \\
\hline 11 & $-0,5$ & $-0,1$ & 0,6 & 9,1 \\
\hline 12 & $-0,5$ & $-0,1$ & 0,6 & 9,1 \\
\hline 13 & $-0,5$ & $-0,1$ & 0,7 & 9,8 \\
\hline 14 & $-0,5$ & $-0,1$ & 0,9 & 10,9 \\
\hline
\end{tabular}


Tabela A59 - Coeficientes de sensibilidade para a pressão de saída a 5000 rpm, mistura 30/70 com 50 kPa de pressão de sistema.

\begin{tabular}{|c|c|c|c|c|}
\hline Índice & $\mathbf{S}_{\mathbf{P} \mathbf{2}}$ & $\mathbf{S}_{\mathbf{P} \mathbf{2} \times} \mathbf{u}_{\mathbf{p}}$ & $\mathbf{1 0 0} \mathbf{x}\left(\mathbf{S}_{\mathbf{P} \mathbf{2}} \mathbf{\mathbf { u } _ { \mathbf { p } }}\right)$ & $\begin{array}{c}\mathbf{2} \\
\text { Contribuição do Transdutor } \\
\mathbf{d e} \text { Pressão da Saída [\%] }\end{array}$ \\
\hline 1 & 1,4 & 0,2 & 6,0 & 92,2 \\
\hline 2 & 1,4 & 0,2 & 6,0 & 92,1 \\
\hline 3 & 1,4 & 0,2 & 6,0 & 92,2 \\
\hline 4 & 1,4 & 0,2 & 6,0 & 92,2 \\
\hline 5 & 1,4 & 0,2 & 6,1 & 92,0 \\
\hline 6 & 1,4 & 0,2 & 6,1 & 92,0 \\
\hline 7 & 1,4 & 0,2 & 6,1 & 91,9 \\
\hline 8 & 1,4 & 0,2 & 6,2 & 91,8 \\
\hline 9 & 1,4 & 0,2 & 6,2 & 91,6 \\
\hline 10 & 1,4 & 0,3 & 6,3 & 91,4 \\
\hline 11 & 1,5 & 0,3 & 6,5 & 90,9 \\
\hline 12 & 1,5 & 0,3 & 6,5 & 90,9 \\
\hline 13 & 1,5 & 0,3 & 6,7 & 90,2 \\
\hline 14 & 1,5 & 0,3 & 7,2 & 89,1 \\
\hline
\end{tabular}

A Tabela A60 trás o cálculo da incerteza combinada para as medições feitas a 1000 rpm, com pressão de sistema de $15 \mathrm{kPa}$, utilizando a mistura de 50\% etilenoglicol e 50\% água como fluido.

Tabela A60 - Incerteza combinada para 1000 rpm, mistura 50/50 com 15 kPa de pressão de sistema.

\begin{tabular}{|c|c|c|c|c|c|}
\hline Índice & $\mathbf{T}\left[{ }^{\circ} \mathbf{C}\right]$ & $\mathbf{P} 1[\mathbf{k P a}]$ & $\mathbf{P} 2[\mathbf{k P a}]$ & $\mathbf{D P}[\mathbf{k P a}]$ & $\begin{array}{c}\mathbf{u}_{\mathbf{c}} \\
{[\%]}\end{array}$ \\
\hline 1 & 85 & 11,5 & 17,5 & 6,0 & 6,1 \\
\hline 2 & 90 & 11,3 & 17,4 & 6,1 & 5,9 \\
\hline 3 & 95 & 11,4 & 17,3 & 5,9 & 6,1 \\
\hline 4 & 97 & 11,2 & 17,0 & 5,8 & 6,1 \\
\hline 5 & 99 & 11,4 & 17,5 & 6,1 & 6,0 \\
\hline 6 & 101 & 11,2 & 17,1 & 5,9 & 6,0 \\
\hline 7 & 103 & 11,0 & 17,1 & 6,1 & 5,8 \\
\hline 8 & 105 & 10,8 & 16,8 & 6,0 & 5,8 \\
\hline 9 & 107 & 11,2 & 17,2 & 6,0 & 6,0 \\
\hline 10 & 109 & 11,4 & 17,5 & 6,1 & 5,9 \\
\hline 11 & 110 & 11,4 & 17,4 & 6,0 & 6,0 \\
\hline
\end{tabular}

As Tabelas A61 e A62 apresentam o cálculo dos coeficientes de sensibilidade das medições das pressões de entrada e saída, 
respectivamente. A primeira coluna referencia a seqüência de medição. A segunda, terceira e quarta coluna contém a informação para cada um dos componentes da Eq. 13. A quinta coluna traz a contribuição para a incerteza combinada.

Tabela A61 - Coeficientes de sensibilidade para a pressão de entrada a 1000 rpm, mistura 50/50 com 15 kPa de pressão de sistema.

\begin{tabular}{|c|c|c|c|c|}
\hline Índice & $S_{P 1}$ & $S_{P 1 x} u_{p}$ & $100 \times\left(S_{P 1 \times} u_{p}\right)^{2}$ & $\begin{array}{l}\text { Contribuição do Transdutor } \\
\text { de Pressão da Entrada[\%] }\end{array}$ \\
\hline 1 & \begin{tabular}{|l|}
$-1,9$ \\
\end{tabular} & $\begin{array}{l}-0,3 \\
\end{array}$ & 11,1 & 30,2 \\
\hline 2 & $-1,9$ & $-0,3$ & 10,4 & 29,7 \\
\hline 3 & $-1,9$ & $-0,3$ & 11,5 & 30,4 \\
\hline 4 & $-1,9$ & $-0,3$ & 11,4 & 30,3 \\
\hline 5 & $-1,9$ & $-0,3$ & 10,6 & 29,8 \\
\hline 6 & $-1,9$ & $-0,3$ & 11,0 & 30,1 \\
\hline 7 & $-1,8$ & $-0,3$ & 9,8 & 29,3 \\
\hline 8 & $-1,8$ & $-0,3$ & 9,7 & 29,2 \\
\hline 9 & $-1,9$ & $-0,3$ & 10,6 & 29,8 \\
\hline 10 & $-1,9$ & $-0,3$ & 10,5 & 29,7 \\
\hline 11 & $-1,9$ & $-0,3$ & 10,9 & 30,0 \\
\hline
\end{tabular}

Tabela A62 - Coeficientes de sensibilidade para a pressão de saída a 1000 rpm, mistura 50/50 com 15 kPa de pressão de sistema.

\begin{tabular}{|c|c|c|c|c|}
\hline Índice & $\mathbf{S}_{\mathbf{P} \mathbf{2}}$ & $\mathbf{S}_{\mathbf{P} \mathbf{2} \times} \mathbf{u}_{\mathbf{p}}$ & $\mathbf{1 0 0} \mathbf{x}\left(\mathbf{S}_{\mathbf{P} \mathbf{x}} \mathbf{\mathbf { u } _ { \mathbf { p } }} \mathbf{)}^{\mathbf{2}}\right.$ & $\begin{array}{c}\text { Contribuição do Transdutor } \\
\text { de Pressão da Saída [\%] }\end{array}$ \\
\hline 1 & 2,9 & 0,5 & 25,8 & 69,8 \\
\hline 2 & 2,9 & 0,5 & 24,6 & 70,3 \\
\hline 3 & 2,9 & 0,5 & 26,3 & 69,6 \\
\hline 4 & 2,9 & 0,5 & 26,2 & 69,7 \\
\hline 5 & 2,9 & 0,5 & 24,9 & 70,2 \\
\hline 6 & 2,9 & 0,5 & 25,6 & 69,9 \\
\hline 7 & 2,8 & 0,5 & 23,8 & 70,7 \\
\hline 8 & 2,8 & 0,5 & 23,6 & 70,8 \\
\hline 9 & 2,9 & 0,5 & 25,0 & 70,2 \\
\hline 10 & 2,9 & 0,5 & 24,8 & 70,3 \\
\hline 11 & 2,9 & 0,5 & 25,5 & 70,0 \\
\hline
\end{tabular}


A Tabela A63 trás o cálculo da incerteza combinada para as medições feitas a $2000 \mathrm{rpm}$, com pressão de sistema de $15 \mathrm{kPa}$, utilizando a mistura de $50 \%$ etilenoglicol e $50 \%$ água como fluido.

Tabela A63 - Incerteza combinada para 2000 rpm, mistura 50/50 com 15 $\mathrm{kPa}$ de pressão de sistema.

\begin{tabular}{|c|c|c|c|c|c|}
\hline Índice & $\mathbf{T}\left[{ }^{\circ} \mathbf{C}\right]$ & $\mathbf{P} 1[\mathbf{k P a}]$ & $\mathbf{P} 2[\mathbf{k P a}]$ & $\mathbf{D P}[\mathbf{k P a}]$ & $\begin{array}{c}\mathbf{u}_{\mathbf{c}} \\
{[\%]}\end{array}$ \\
\hline 1 & 85 & 11,9 & 32,2 & 20,3 & 2,9 \\
\hline 2 & 90 & 12,0 & 32,3 & 20,3 & 3,0 \\
\hline 3 & 95 & 11,8 & 32,0 & 20,2 & 2,9 \\
\hline 4 & 97 & 11,8 & 32,1 & 20,3 & 2,9 \\
\hline 5 & 99 & 12,0 & 32,0 & 20,0 & 3,0 \\
\hline 6 & 101 & 11,9 & 32,1 & 20,2 & 3,0 \\
\hline 7 & 103 & 12,0 & 32,2 & 20,2 & 3,0 \\
\hline 8 & 105 & 12,1 & 32,0 & 19,9 & 3,0 \\
\hline 9 & 107 & 12,4 & 32,3 & 19,9 & 3,0 \\
\hline 10 & 109 & 12,0 & 32,1 & 20,1 & 3,0 \\
\hline 11 & 111 & 11,8 & 31,7 & 19,9 & 3,0 \\
\hline
\end{tabular}

As Tabelas A64 e A65 apresentam o cálculo dos coeficientes de sensibilidade das medições das pressões de entrada e saída, respectivamente. A primeira coluna referencia a seqüência de medição. A segunda, terceira e quarta coluna contém a informação para cada um dos componentes da Eq. 13. A quinta coluna traz a contribuição para a incerteza combinada. 
Tabela A64 - Coeficientes de sensibilidade para a pressão de entrada a 2000 rpm, mistura 50/50 com 15 kPa de pressão de sistema.

\begin{tabular}{|c|c|c|c|c|}
\hline Índice & $\mathbf{S}_{\mathbf{p} \mathbf{1}}$ & $\mathbf{S}_{\mathbf{p} \mathbf{1} \mathbf{x}} \mathbf{u}_{\mathbf{p}}$ & $\mathbf{1 0 0} \mathbf{x}\left(\mathbf{S}_{\mathbf{p} \mathbf{x} \mathbf{x}} \mathbf{u}_{\mathbf{p}}\right)^{\mathbf{2}}$ & $\begin{array}{c}\text { Contribuição do Transdutor } \\
\text { de Pressão da Entrada[\%] }\end{array}$ \\
\hline 1 & $-0,6$ & $-0,1$ & 1,0 & 12,0 \\
\hline 2 & $-0,6$ & $-0,1$ & 1,1 & 12,1 \\
\hline 3 & $-0,6$ & $-0,1$ & 1,0 & 12,0 \\
\hline 4 & $-0,6$ & $-0,1$ & 1,0 & 11,9 \\
\hline 5 & $-0,6$ & $-0,1$ & 1,1 & 12,4 \\
\hline 6 & $-0,6$ & $-0,1$ & 1,1 & 12,1 \\
\hline 7 & $-0,6$ & $-0,1$ & 1,1 & 12,2 \\
\hline 8 & $-0,6$ & $-0,1$ & 1,1 & 12,5 \\
\hline 9 & $-0,6$ & $-0,1$ & 1,2 & 12,8 \\
\hline 10 & $-0,6$ & $-0,1$ & 1,1 & 12,3 \\
\hline 11 & $-0,6$ & $-0,1$ & 1,1 & 12,2 \\
\hline
\end{tabular}

Tabela A65 - Coeficientes de sensibilidade para a pressão de saída a 2000 rpm, mistura 50/50 com 15 kPa de pressão de sistema.

\begin{tabular}{|c|c|c|c|c|}
\hline Índice & $S_{P 2}$ & $S_{P 2 \times} u_{p}$ & $100 \times\left(S_{P 2} \times u_{p}\right)^{2}$ & $\begin{array}{l}\text { Contribuição do Transdutor } \\
\text { de Pressão da Saída [\%] }\end{array}$ \\
\hline 1 & 1,6 & 0,3 & 7,6 & 88,0 \\
\hline 2 & 1,6 & 0,3 & 7,7 & 87,9 \\
\hline 3 & 1,6 & 0,3 & 7,6 & 88,0 \\
\hline 4 & 1,6 & 0,3 & 7,6 & 88,1 \\
\hline 5 & 1,6 & 0,3 & 7,8 & 87,6 \\
\hline 6 & 1,6 & 0,3 & 7,7 & 87,9 \\
\hline 7 & 1,6 & 0,3 & 7,7 & 87,8 \\
\hline 8 & 1,6 & 0,3 & 7,8 & 87,5 \\
\hline 9 & 1,6 & 0,3 & 8,0 & 87,2 \\
\hline 10 & 1,6 & 0,3 & 7,7 & 87,7 \\
\hline 11 & 1,6 & 0,3 & 7,7 & 87,8 \\
\hline
\end{tabular}

A Tabela A66 trás o cálculo da incerteza combinada para as medições feitas a 3000 rpm, com pressão de sistema de $15 \mathrm{kPa}$, utilizando a mistura de 50\% etilenoglicol e 50\% água como fluido. 
Tabela A66 - Incerteza combinada para 3000 rpm, mistura 50/50 com 15 kPa de pressão de sistema.

\begin{tabular}{|c|c|c|c|c|c|}
\hline Índice & $\mathbf{T}\left[{ }^{\circ} \mathbf{C}\right]$ & $\mathbf{P 1}[\mathbf{k P a}]$ & $\mathbf{P 2}[\mathbf{k P a}]$ & $\mathbf{D P}[\mathbf{k P a}]$ & $\begin{array}{c}\mathbf{u}_{\mathbf{c}} \\
{[\%]}\end{array}$ \\
\hline 1 & 85 & 15,3 & 57,3 & 42,0 & 2,5 \\
\hline 2 & 90 & 15,4 & 57,5 & 42,1 & 2,5 \\
\hline 3 & 95 & 15,6 & 57,9 & 42,3 & 2,5 \\
\hline 4 & 97 & 15,1 & 57,2 & 42,1 & 2,4 \\
\hline 5 & 99 & 15,8 & 58,1 & 42,3 & 2,5 \\
\hline 6 & 101 & 15,1 & 57,7 & 42,6 & 2,4 \\
\hline 7 & 103 & 15,9 & 58,0 & 42,1 & 2,5 \\
\hline 8 & 105 & 15,7 & 57,7 & 42,0 & 2,5 \\
\hline 9 & 107 & 15,9 & 58,3 & 42,4 & 2,5 \\
\hline 10 & 109 & 15,5 & 56,1 & 40,6 & 2,5 \\
\hline 11 & 111 & 15,8 & 51,5 & 35,7 & 2,6 \\
\hline
\end{tabular}

As Tabelas A67 e A68 apresentam o cálculo dos coeficientes de sensibilidade das medições das pressões de entrada e saída, respectivamente. A primeira coluna referencia a seqüência de medição. A segunda, terceira e quarta coluna contém a informação para cada um dos componentes da Eq. 13. A quinta coluna traz a contribuição para a incerteza combinada.

Tabela A67 - Coeficientes de sensibilidade para a pressão de entrada a $3000 \mathrm{rpm}$, mistura 50/50 com $15 \mathrm{kPa}$ de pressão de sistema.

\begin{tabular}{|c|c|c|c|c|}
\hline Índice & $S_{P_{1}}$ & $S_{P 1 \times} u_{p}$ & $100 \times\left(S_{P 1 \times} u_{p}\right)^{2}$ & $\begin{array}{l}\text { Contribuição do Transdutor } \\
\text { de Pressão da Entrada[\%] }\end{array}$ \\
\hline 1 & $-0,4$ & $-0,1$ & 0,4 & 6,7 \\
\hline 2 & $-0,4$ & $-0,1$ & 0,4 & 6,7 \\
\hline 3 & $-0,4$ & $-0,1$ & 0,4 & 6,8 \\
\hline 4 & $-0,4$ & $-0,1$ & 0,4 & 6,5 \\
\hline 5 & $-0,4$ & $-0,1$ & 0,4 & 6,9 \\
\hline 6 & $-0,4$ & $-0,1$ & 0,4 & 6,4 \\
\hline 7 & $-0,4$ & $-0,1$ & 0,4 & 7,0 \\
\hline 8 & $-0,4$ & $-0,1$ & 0,4 & 6,9 \\
\hline 9 & $-0,4$ & $-0,1$ & 0,4 & 6,9 \\
\hline 10 & $-0,4$ & $-0,1$ & 0,4 & 7,1 \\
\hline 11 & $-0,4$ & $-0,1$ & 0,6 & 8,6 \\
\hline
\end{tabular}


Tabela A68 - Coeficientes de sensibilidade para a pressão de saída a 3000 rpm, mistura 50/50 com 15 kPa de pressão de sistema.

\begin{tabular}{|c|c|c|c|c|}
\hline Índice & $\mathrm{S}_{\mathrm{P} 2}$ & $S_{P 2 \times} u_{p}$ & $100 \times\left(S_{P 2} \times u_{p}\right)^{2}$ & $\begin{array}{l}\text { Contribuição do Transdutor } \\
\text { de Pressão da Saída [\%] }\end{array}$ \\
\hline 1 & 1,4 & 0,2 & 5,6 & 93,3 \\
\hline 2 & 1,4 & 0,2 & 5,6 & 93,3 \\
\hline 3 & 1,4 & 0,2 & 5,7 & 93,2 \\
\hline 4 & 1,4 & 0,2 & 5,6 & 93,5 \\
\hline 5 & 1,4 & 0,2 & 5,7 & 93,1 \\
\hline 6 & 1,4 & 0,2 & 5,6 & 93,6 \\
\hline 7 & 1,4 & 0,2 & 5,8 & 93,0 \\
\hline 8 & 1,4 & 0,2 & 5,7 & 93,1 \\
\hline 9 & 1,4 & 0,2 & 5,7 & 93,1 \\
\hline 10 & 1,4 & 0,2 & 5,8 & 92,9 \\
\hline 11 & 1,4 & 0,3 & 6,3 & 91,4 \\
\hline
\end{tabular}

A Tabela A69 trás o cálculo da incerteza combinada para as medições feitas a 4000 rpm, com pressão de sistema de $15 \mathrm{kPa}$, utilizando a mistura de 50\% etilenoglicol e 50\% água como fluido.

Tabela A69 - Incerteza combinada para 4000 rpm, mistura 50/50 com 15 kPa de pressão de sistema.

\begin{tabular}{|c|c|c|c|c|c|}
\hline Índice & $\mathbf{T}\left[{ }^{\circ} \mathbf{C}\right]$ & $\mathbf{P} 1[\mathbf{k P a}]$ & $\mathbf{P} 2 \mathbf{[ k P a}]$ & $\mathbf{D P}[\mathbf{k P a}]$ & $\begin{array}{c}\mathbf{u}_{\mathbf{c}} \\
{[\%]}\end{array}$ \\
\hline 1 & 85 & 12,0 & 87,8 & 75,8 & 2,0 \\
\hline 2 & 90 & 11,9 & 87,6 & 75,7 & 2,0 \\
\hline 3 & 95 & 11,8 & 87,4 & 75,6 & 2,0 \\
\hline 4 & 97 & 12,0 & 87,8 & 75,8 & 2,0 \\
\hline 5 & 99 & 12,0 & 87,9 & 75,9 & 2,0 \\
\hline 6 & 101 & 11,9 & 87,6 & 75,7 & 2,0 \\
\hline 7 & 103 & 12,1 & 88,1 & 76,0 & 2,0 \\
\hline 8 & 105 & 11,8 & 83,6 & 71,8 & 2,0 \\
\hline 9 & 107 & 11,0 & 75,7 & 64,7 & 2,1 \\
\hline 10 & 110 & 10,4 & 67,4 & 57,0 & 2,1 \\
\hline
\end{tabular}

As Tabelas A70 e A71 apresentam o cálculo dos coeficientes de sensibilidade das medições das pressões de entrada e saída, respectivamente. A primeira coluna referencia a seqüência de medição. $A$ segunda, terceira e quarta coluna contém a informação para cada um dos 
componentes da Eq. 13. A quinta coluna traz a contribuição para a incerteza combinada.

Tabela A70 - Coeficientes de sensibilidade para a pressão de entrada a 4000 rpm, mistura 50/50 com 15 kPa de pressão de sistema.

\begin{tabular}{|c|c|c|c|c|}
\hline Índice & $S_{P_{1}}$ & $S_{p 1 \times} u_{p}$ & $100 \times\left(S_{P_{1} \times} u_{p}\right)^{2}$ & $\begin{array}{l}\text { Contribuição do Transdutor } \\
\text { de Pressão da Entrada[\%] }\end{array}$ \\
\hline 1 & $-0,2$ & 0,0 & 0,1 & 1,8 \\
\hline 2 & $-0,2$ & 0,0 & 0,1 & 1,8 \\
\hline 3 & $-0,2$ & 0,0 & 0,1 & 1,8 \\
\hline 4 & $-0,2$ & 0,0 & 0,1 & 1,8 \\
\hline 5 & $-0,2$ & 0,0 & 0,1 & 1,8 \\
\hline 6 & $-0,2$ & 0,0 & 0,1 & 1,8 \\
\hline 7 & $-0,2$ & 0,0 & 0,1 & 1,9 \\
\hline 8 & $-0,2$ & 0,0 & 0,1 & 2,0 \\
\hline 9 & $-0,2$ & 0,0 & 0,1 & 2,1 \\
\hline 10 & $-0,2$ & 0,0 & 0,1 & 2,3 \\
\hline
\end{tabular}

Tabela A71 - Coeficientes de sensibilidade para a pressão de saída a $4000 \mathrm{rpm}$, mistura 50/50 com $15 \mathrm{kPa}$ de pressão de sistema.

\begin{tabular}{|c|c|c|c|c|}
\hline Índice & $S_{p 2}$ & $S_{p 2 \times} u_{p}$ & $100 \times\left(S_{P_{2} \times} u_{p}\right)^{2}$ & $\begin{array}{l}\text { Contribuição do Transdutor } \\
\text { de Pressão da Saída [\%] }\end{array}$ \\
\hline 1 & 1,2 & 0,2 & 4,1 & 98,2 \\
\hline 2 & 1,2 & 0,2 & 4,1 & 98,2 \\
\hline 3 & 1,2 & 0,2 & 4,0 & 98,2 \\
\hline 4 & 1,2 & 0,2 & 4,1 & 98,2 \\
\hline 5 & 1,2 & 0,2 & 4,1 & 98,2 \\
\hline 6 & 1,2 & 0,2 & 4,1 & 98,2 \\
\hline 7 & 1,2 & 0,2 & 4,1 & 98,1 \\
\hline 8 & 1,2 & 0,2 & 4,1 & 98,0 \\
\hline 9 & 1,2 & 0,2 & 4,1 & 97,9 \\
\hline 10 & 1,2 & 0,2 & 4,2 & 97,7 \\
\hline
\end{tabular}

A Tabela A72 trás o cálculo da incerteza combinada para as medições feitas a $5000 \mathrm{rpm}$, com pressão de sistema de $15 \mathrm{kPa}$, utilizando a mistura de $50 \%$ etilenoglicol e 50\% água como fluido. 
Tabela A72 - Incerteza combinada para 5000 rpm, mistura 50/50 com 15 kPa de pressão de sistema.

\begin{tabular}{|c|c|c|c|c|c|}
\hline Índice & $\mathbf{T}\left[{ }^{\circ} \mathbf{C}\right]$ & $\mathbf{P}$ [ $[\mathbf{k P a}]$ & $\mathbf{P} 2[\mathbf{k P a}]$ & $\mathbf{D P}[\mathbf{k P a}]$ & $\begin{array}{c}\mathbf{u}_{\mathbf{c}} \\
{[\%]}\end{array}$ \\
\hline 1 & 85 & 12,1 & 110,1 & 98,0 & 2,0 \\
\hline 2 & 90 & 12,2 & 110,0 & 97,8 & 2,0 \\
\hline 3 & 93 & 12,0 & 109,7 & 97,7 & 2,0 \\
\hline 4 & 95 & 12,1 & 110,1 & 98,0 & 2,0 \\
\hline 5 & 97 & 11,8 & 109,1 & 97,3 & 2,0 \\
\hline 6 & 99 & 11,9 & 110,1 & 98,2 & 2,0 \\
\hline 7 & 101 & 12,2 & 108,8 & 96,6 & 2,0 \\
\hline 8 & 103 & 12,5 & 105,4 & 92,9 & 2,0 \\
\hline 9 & 105 & 11,9 & 97,2 & 85,3 & 2,0 \\
\hline 10 & 107 & 11,0 & 82,1 & 71,1 & 2,0 \\
\hline 11 & 110 & 9,5 & 67,5 & 58,0 & 2,0 \\
\hline
\end{tabular}

As Tabelas A73 e A74 apresentam o cálculo dos coeficientes de sensibilidade das medições das pressões de entrada e saída, respectivamente. A primeira coluna referencia a seqüência de medição. A segunda, terceira e quarta coluna contém a informação para cada um dos componentes da Eq. 13. A quinta coluna traz a contribuição para a incerteza combinada.

Tabela A73 - Coeficientes de sensibilidade para a pressão de entrada a $5000 \mathrm{rpm}$, mistura 50/50 com $15 \mathrm{kPa}$ de pressão de sistema.

\begin{tabular}{|c|c|c|c|c|}
\hline Índice & $\mathbf{S}_{\mathbf{P} \mathbf{1}}$ & $\mathbf{S}_{\mathbf{P} \mathbf{1} \mathbf{x}} \mathbf{u}_{\mathbf{p}}$ & $\mathbf{1 0 0} \mathbf{x}\left(\mathbf{S}_{\mathbf{p} \mathbf{x}} \mathbf{\mathbf { u } _ { \mathbf { p } }}\right)$ & $\begin{array}{c}\mathbf{2} \\
\text { Contribuição do Transdutor } \\
\mathbf{d e ~ P r e s s a ̃ o ~ d a ~ E n t r a d a [ \% ] ~}\end{array}$ \\
\hline 1 & $-0,1$ & 0,0 & 0,0 & 1,2 \\
\hline 2 & $-0,1$ & 0,0 & 0,0 & 1,2 \\
\hline 3 & $-0,1$ & 0,0 & 0,0 & 1,2 \\
\hline 4 & $-0,1$ & 0,0 & 0,0 & 1,2 \\
\hline 5 & $-0,1$ & 0,0 & 0,0 & 1,2 \\
\hline 6 & $-0,1$ & 0,0 & 0,0 & 1,2 \\
\hline 7 & $-0,1$ & 0,0 & 0,0 & 1,2 \\
\hline 8 & $-0,1$ & 0,0 & 0,1 & 1,4 \\
\hline 9 & $-0,1$ & 0,0 & 0,1 & 1,5 \\
\hline 10 & $-0,2$ & 0,0 & 0,1 & 1,8 \\
\hline 11 & $-0,2$ & 0,0 & 0,1 & 1,9 \\
\hline
\end{tabular}


Tabela A74 - Coeficientes de sensibilidade para a pressão de saída a 5000 rpm, mistura 50/50 com 15 kPa de pressão de sistema.

\begin{tabular}{|c|c|c|c|c|}
\hline Índice & $S_{P 2}$ & $S_{P 2 \times} u_{p}$ & $100 \times\left(S_{P 2 \times} u_{p}\right)^{2}$ & $\begin{array}{l}\text { Contribuição do Transdutor } \\
\text { de Pressão da Saída [\%] }\end{array}$ \\
\hline 1 & 1,1 & 0,2 & 3,8 & 98,8 \\
\hline 2 & 1,1 & 0,2 & 3,8 & 98,8 \\
\hline 3 & 1,1 & 0,2 & 3,8 & 98,8 \\
\hline 4 & 1,1 & 0,2 & 3,8 & 98,8 \\
\hline 5 & 1,1 & 0,2 & 3,8 & 98,8 \\
\hline 6 & 1,1 & 0,2 & 3,8 & 98,8 \\
\hline 7 & 1,1 & 0,2 & 3,8 & 98,8 \\
\hline 8 & 1,1 & 0,2 & 3,9 & 98,6 \\
\hline 9 & 1,1 & 0,2 & 3,9 & 98,5 \\
\hline 10 & 1,2 & 0,2 & 4,0 & 98,2 \\
\hline 11 & 1,2 & 0,2 & 4,1 & 98,1 \\
\hline
\end{tabular}

A Tabela A75 trás o cálculo da incerteza combinada para as medições feitas a 1000 rpm, com pressão de sistema de $50 \mathrm{kPa}$, utilizando a mistura de 50\% etilenoglicol e 50\% água como fluido.

Tabela A75 - Incerteza combinada para 1000 rpm, mistura 50/50 com 50 kPa de pressão de sistema.

\begin{tabular}{|c|c|c|c|c|c|}
\hline Índice & $\mathbf{T}\left[{ }^{\circ} \mathbf{C}\right]$ & $\mathbf{P}$ [ $[\mathbf{k P a}]$ & $\mathbf{P 2}[\mathbf{k P a}]$ & $\mathbf{D P}[\mathbf{k P a}]$ & $\begin{array}{c}\mathbf{u}_{\mathbf{c}} \\
{[\%]}\end{array}$ \\
\hline 1 & 85 & 43,5 & 50,6 & 7,1 & 16,4 \\
\hline 2 & 90 & 43,7 & 50,7 & 7,0 & 16,6 \\
\hline 3 & 95 & 43,6 & 50,8 & 7,2 & 16,1 \\
\hline 4 & 97 & 43,7 & 50,9 & 7,2 & 16,3 \\
\hline 5 & 99 & 43,5 & 50,9 & 7,4 & 15,8 \\
\hline 6 & 101 & 43,3 & 50,5 & 7,2 & 16,1 \\
\hline 7 & 103 & 43,2 & 50,6 & 7,4 & 15,6 \\
\hline 8 & 105 & 42,8 & 50,3 & 7,5 & 15,4 \\
\hline 9 & 107 & 43,7 & 50,9 & 7,2 & 16,2 \\
\hline 10 & 109 & 43,5 & 51,1 & 7,6 & 15,4 \\
\hline 11 & 110 & 43,4 & 50,8 & 7,4 & 15,7 \\
\hline
\end{tabular}

As Tabelas A76 e A77 apresentam o cálculo dos coeficientes de sensibilidade das medições das pressões de entrada e saída, respectivamente. A primeira coluna referencia a seqüência de medição. $A$ segunda, terceira e quarta coluna contém a informação para cada um dos 
componentes da Eq. 13. A quinta coluna traz a contribuição para a incerteza combinada.

Tabela A76 - Coeficientes de sensibilidade para a pressão de entrada a $1000 \mathrm{rpm}$, mistura 50/50 com $50 \mathrm{kPa}$ de pressão de sistema.

\begin{tabular}{|c|c|c|c|c|}
\hline Índice & $\mathbf{S}_{\mathbf{P} \mathbf{1}}$ & $\mathbf{S}_{\mathbf{P} \mathbf{1}} \mathbf{u}_{\mathbf{p}}$ & $\mathbf{1 0 0} \mathbf{x}\left(\mathbf{S}_{\mathbf{P} \mathbf{1}} \mathbf{\mathbf { u } _ { \mathbf { p } }}\right)$ & $\begin{array}{c}\mathbf{2} \\
\text { Contribuição do Transdutor } \\
\text { de Pressão da Entrada[\%] }\end{array}$ \\
\hline 1 & $-6,1$ & $-1,1$ & 113,6 & 42,5 \\
\hline 2 & $-6,2$ & $-1,1$ & 118,0 & 42,6 \\
\hline 3 & $-6,0$ & $-1,1$ & 110,3 & 42,4 \\
\hline 4 & $-6,1$ & $-1,1$ & 112,3 & 42,5 \\
\hline 5 & $-5,9$ & $-1,0$ & 106,0 & 42,3 \\
\hline 6 & $-6,0$ & $-1,0$ & 109,5 & 42,4 \\
\hline 7 & $-5,8$ & $-1,0$ & 103,2 & 42,2 \\
\hline 8 & $-5,7$ & $-1,0$ & 99,3 & 42,0 \\
\hline 9 & $-6,1$ & $-1,1$ & 111,5 & 42,4 \\
\hline 10 & $-5,7$ & $-1,0$ & 99,8 & 42,0 \\
\hline 11 & $-5,8$ & $-1,0$ & 103,4 & 42,2 \\
\hline
\end{tabular}

Tabela A77 - Coeficientes de sensibilidade para a pressão de saída a $1000 \mathrm{rpm}$, mistura 50/50 com $50 \mathrm{kPa}$ de pressão de sistema.

\begin{tabular}{|c|c|c|c|c|}
\hline Índice & $\mathrm{S}_{\mathrm{P} 2}$ & $S_{P 2 \times} u_{p}$ & $100 \times\left(S_{P 2} \times u_{p}\right)^{2}$ & $\begin{array}{l}\text { Contribuição do Transdutor } \\
\text { de Pressão da Saída [\%] }\end{array}$ \\
\hline 1 & 7,1 & 1,2 & 153,8 & 57,5 \\
\hline 2 & 7,2 & 1,3 & 158,8 & 57,4 \\
\hline 3 & 7,0 & 1,2 & 149,8 & 57,6 \\
\hline 4 & 7,1 & 1,2 & 152,2 & 57,5 \\
\hline 5 & 6,9 & 1,2 & 144,9 & 57,7 \\
\hline 6 & 7,0 & 1,2 & 148,9 & 57,6 \\
\hline 7 & 6,8 & 1,2 & 141,6 & 57,8 \\
\hline 8 & 6,7 & 1,2 & 137,0 & 58,0 \\
\hline 9 & 7,1 & 1,2 & 151,3 & 57,6 \\
\hline 10 & 6,7 & 1,2 & 137,6 & 58,0 \\
\hline 11 & 6,8 & 1,2 & 141,9 & 57,8 \\
\hline
\end{tabular}

A Tabela A78 trás o cálculo da incerteza combinada para as medições feitas a $2000 \mathrm{rpm}$, com pressão de sistema de $50 \mathrm{kPa}$, utilizando a mistura de $50 \%$ etilenoglicol e $50 \%$ água como fluido. 
Tabela A78 - Incerteza combinada para 2000 rpm, mistura 50/50 com 50 kPa de pressão de sistema.

\begin{tabular}{|c|c|c|c|c|c|}
\hline Índice & $\mathbf{T}\left[{ }^{\circ} \mathbf{C}\right]$ & $\mathbf{P}$ [ $[\mathbf{k P a}]$ & $\mathbf{P} 2[\mathbf{k P a}]$ & $\mathbf{D P}[\mathbf{k P a}]$ & $\begin{array}{c}\mathbf{u}_{\mathbf{c}} \\
{[\%]}\end{array}$ \\
\hline 1 & 85 & 12,0 & 32,8 & 20,8 & 2,9 \\
\hline 2 & 90 & 11,9 & 32,8 & 20,9 & 2,9 \\
\hline 3 & 95 & 11,8 & 32,7 & 20,9 & 2,9 \\
\hline 4 & 97 & 11,8 & 32,9 & 21,1 & 2,9 \\
\hline 5 & 99 & 12,0 & 32,7 & 20,7 & 2,9 \\
\hline 6 & 101 & 11,9 & 32,3 & 20,4 & 2,9 \\
\hline 7 & 103 & 12,0 & 32,9 & 20,9 & 2,9 \\
\hline 8 & 105 & 12,1 & 33,1 & 21,0 & 2,9 \\
\hline 9 & 107 & 12,4 & 33,3 & 20,9 & 3,0 \\
\hline 10 & 109 & 12,0 & 32,5 & 20,5 & 2,9 \\
\hline 11 & 110 & 11,8 & 32,6 & 20,8 & 2,9 \\
\hline
\end{tabular}

As Tabelas A79 e A80 apresentam o cálculo dos coeficientes de sensibilidade das medições das pressões de entrada e saída, respectivamente. A primeira coluna referencia a seqüência de medição. A segunda, terceira e quarta coluna contém a informação para cada um dos componentes da Eq. 13. A quinta coluna traz a contribuição para a incerteza combinada.

Tabela A79 - Coeficientes de sensibilidade para a pressão de entrada a 2000 rpm, mistura 50/50 com $50 \mathrm{kPa}$ de pressão de sistema.

\begin{tabular}{|c|c|c|c|c|}
\hline Índice & $S_{P 1}$ & $S_{P 1 x} u_{p}$ & $100 \times\left(S_{p 1 \times} u_{p}\right)^{2}$ & $\begin{array}{l}\text { Contribuição do Transdutor } \\
\text { de Pressão da Entrada[\%] }\end{array}$ \\
\hline 1 & $-0,6$ & $-0,1$ & 1,0 & 11,8 \\
\hline 2 & $-0,6$ & $-0,1$ & 1,0 & 11,6 \\
\hline 3 & $-0,6$ & $-0,1$ & 1,0 & 11,5 \\
\hline 4 & $-0,6$ & $-0,1$ & 1,0 & 11,4 \\
\hline 5 & $-0,6$ & $-0,1$ & 1,0 & 11,9 \\
\hline 6 & $-0,6$ & $-0,1$ & 1,0 & 11,9 \\
\hline 7 & $-0,6$ & $-0,1$ & 1,0 & 11,8 \\
\hline 8 & $-0,6$ & $-0,1$ & 1,0 & 11,8 \\
\hline 9 & $-0,6$ & $-0,1$ & 1,1 & 12,2 \\
\hline 10 & $-0,6$ & $-0,1$ & 1,0 & 12,0 \\
\hline 11 & $-0,6$ & $-0,1$ & 1,0 & 11,6 \\
\hline
\end{tabular}


Tabela A80 - Coeficientes de sensibilidade para a pressão de saída a 2000 rpm, mistura 50/50 com 50 kPa de pressão de sistema.

\begin{tabular}{|c|c|c|c|c|}
\hline Índice & $S_{P 2}$ & $S_{P 2 \times} u_{p}$ & $100 \times\left(S_{P 2 \times} u_{p}\right)^{2}$ & $\begin{array}{l}\text { Contribuição do Transdutor } \\
\text { de Pressão da Saída [\%] }\end{array}$ \\
\hline 1 & 1,6 & 0,3 & 7,5 & 88,2 \\
\hline 2 & 1,6 & 0,3 & 7,5 & 88,4 \\
\hline 3 & 1,6 & 0,3 & 7,4 & 88,5 \\
\hline 4 & 1,6 & 0,3 & 7,4 & 88,6 \\
\hline 5 & 1,6 & 0,3 & 7,6 & 88,1 \\
\hline 6 & 1,6 & 0,3 & 7,6 & 88,1 \\
\hline 7 & 1,6 & 0,3 & 7,5 & 88,2 \\
\hline 8 & 1,6 & 0,3 & 7,5 & 88,2 \\
\hline 9 & 1,6 & 0,3 & 7,7 & 87,8 \\
\hline 10 & 1,6 & 0,3 & 7,6 & 88,0 \\
\hline 11 & 1,6 & 0,3 & 7,4 & 88,4 \\
\hline
\end{tabular}

A Tabela A81 trás o cálculo da incerteza combinada para as medições feitas a 3000 rpm, com pressão de sistema de $50 \mathrm{kPa}$, utilizando a mistura de $50 \%$ etilenoglicol e 50\% água como fluido.

Tabela A81 - Incerteza combinada para 3000 rpm, mistura 50/50 com 50 kPa de pressão de sistema.

\begin{tabular}{|c|c|c|c|c|c|}
\hline Índice & $\mathbf{T}\left[{ }^{\circ} \mathbf{C}\right]$ & $\mathbf{P 1}[\mathbf{k P a}]$ & $\mathbf{P 2}[\mathbf{k P a}]$ & $\mathbf{D P}[\mathbf{k P a}]$ & $\begin{array}{c}\mathbf{u}_{\mathbf{c}} \\
{[\%]}\end{array}$ \\
\hline 1 & 85 & 11,9 & 55,8 & 43,9 & 2,3 \\
\hline 2 & 90 & 12,1 & 56,2 & 44,1 & 2,3 \\
\hline 3 & 95 & 12,0 & 55,9 & 43,9 & 2,3 \\
\hline 4 & 97 & 12,1 & 55,5 & 43,4 & 2,3 \\
\hline 5 & 99 & 11,8 & 55,7 & 43,9 & 2,3 \\
\hline 6 & 101 & 12,1 & 56,1 & 44,0 & 2,3 \\
\hline 7 & 103 & 11,9 & 55,7 & 43,8 & 2,3 \\
\hline 8 & 105 & 11,7 & 54,7 & 43,0 & 2,3 \\
\hline 9 & 107 & 12,0 & 55,4 & 43,4 & 2,3 \\
\hline 10 & 109 & 11,7 & 54,7 & 43,0 & 2,3 \\
\hline 11 & 110 & 16,8 & 59,1 & 42,3 & 2,5 \\
\hline
\end{tabular}

As Tabelas A82 e A83 apresentam o cálculo dos coeficientes de sensibilidade das medições das pressões de entrada e saída, respectivamente. A primeira coluna referencia a seqüência de medição. $A$ segunda, terceira e quarta coluna contém a informação para cada um dos 
componentes da Eq. 13. A quinta coluna traz a contribuição para a incerteza combinada.

Tabela A82 - Coeficientes de sensibilidade para a pressão de entrada a $3000 \mathrm{rpm}$, mistura 50/50 com $50 \mathrm{kPa}$ de pressão de sistema.

\begin{tabular}{|c|c|c|c|c|}
\hline Índice & $S_{P_{1}}$ & $S_{p 1 \times} u_{p}$ & $100 \times\left(S_{P_{1} \times} u_{p}\right)^{2}$ & $\begin{array}{l}\text { Contribuição do Transdutor } \\
\text { de Pressão da Entrada[\%] }\end{array}$ \\
\hline 1 & $-0,3$ & 0,0 & 0,2 & 4,4 \\
\hline 2 & $-0,3$ & 0,0 & 0,2 & 4,4 \\
\hline 3 & $-0,3$ & 0,0 & 0,2 & 4,4 \\
\hline 4 & $-0,3$ & 0,0 & 0,2 & 4,5 \\
\hline 5 & $-0,3$ & 0,0 & 0,2 & 4,3 \\
\hline 6 & $-0,3$ & 0,0 & 0,2 & 4,4 \\
\hline 7 & $-0,3$ & 0,0 & 0,2 & 4,4 \\
\hline 8 & $-0,3$ & 0,0 & 0,2 & 4,4 \\
\hline 9 & $-0,3$ & 0,0 & 0,2 & 4,5 \\
\hline 10 & $-0,3$ & 0,0 & 0,2 & 4,4 \\
\hline 11 & $-0,4$ & $-0,1$ & 0,5 & 7,5 \\
\hline
\end{tabular}

Tabela A83 - Coeficientes de sensibilidade para a pressão de entrada a 3000 rpm, mistura 50/50 com $50 \mathrm{kPa}$ de pressão de sistema.

\begin{tabular}{|c|c|c|c|c|}
\hline Índice & $S_{P 2}$ & $S_{p 2 x} u_{p}$ & $100 \times\left(S_{P_{2} \times} u_{p}\right)^{2}$ & $\begin{array}{l}\text { Contribuição do Transdutor } \\
\text { de Pressão da Saída [\%] }\end{array}$ \\
\hline 1 & 1,3 & 0,2 & 4,9 & 95,6 \\
\hline 2 & 1,3 & 0,2 & 4,9 & 95,6 \\
\hline 3 & 1,3 & 0,2 & 4,9 & 95,6 \\
\hline 4 & 1,3 & 0,2 & 4,9 & 95,5 \\
\hline 5 & 1,3 & 0,2 & 4,9 & 95,7 \\
\hline 6 & 1,3 & 0,2 & 4,9 & 95,6 \\
\hline 7 & 1,3 & 0,2 & 4,9 & 95,6 \\
\hline 8 & 1,3 & 0,2 & 4,9 & 95,6 \\
\hline 9 & 1,3 & 0,2 & 4,9 & 95,5 \\
\hline 10 & 1,3 & 0,2 & 4,9 & 95,6 \\
\hline 11 & 1,4 & 0,2 & 5,9 & 92,5 \\
\hline
\end{tabular}

A Tabela A84 trás o cálculo da incerteza combinada para as medições feitas a $4000 \mathrm{rpm}$, com pressão de sistema de $50 \mathrm{kPa}$, utilizando a mistura de $50 \%$ etilenoglicol e $50 \%$ água como fluido. 
Tabela A84 - Incerteza combinada para 4000 rpm, mistura 50/50 com 50 kPa de pressão de sistema.

\begin{tabular}{|c|c|c|c|c|c|}
\hline Índice & $\mathbf{T}\left[{ }^{\circ} \mathbf{C}\right]$ & $\mathbf{P} 1 \mathbf{[ k P a}]$ & $\mathbf{P} 2[\mathbf{k P a}]$ & $\mathbf{D P}[\mathbf{k P a}]$ & $\begin{array}{c}\mathbf{u}_{\mathbf{c}} \\
{[\mathbf{\%}]}\end{array}$ \\
\hline 1 & 85 & 45,4 & 120,3 & 74,9 & 3,0 \\
\hline 2 & 90 & 45,3 & 120,1 & 74,8 & 3,0 \\
\hline 3 & 93 & 45,6 & 120,5 & 74,9 & 3,0 \\
\hline 4 & 95 & 45,5 & 120,2 & 74,7 & 3,0 \\
\hline 5 & 97 & 45,1 & 120,1 & 75,0 & 3,0 \\
\hline 6 & 99 & 44,8 & 119,4 & 74,6 & 3,0 \\
\hline 7 & 101 & 47,8 & 121,7 & 73,9 & 3,1 \\
\hline 8 & 103 & 45,6 & 120,3 & 74,7 & 3,0 \\
\hline 9 & 105 & 45,4 & 120,1 & 74,7 & 3,0 \\
\hline 10 & 107 & 48,0 & 119,8 & 71,8 & 3,1 \\
\hline
\end{tabular}

As Tabelas A85 e A86 apresentam o cálculo dos coeficientes de sensibilidade das medições das pressões de entrada e saída, respectivamente. A primeira coluna referencia a seqüência de medição. A segunda, terceira e quarta coluna contém a informação para cada um dos componentes da Eq. 13. A quinta coluna traz a contribuição para a incerteza combinada.

Tabela A85 - Coeficientes de sensibilidade para a pressão de entrada a 4000 rpm, mistura 50/50 com $50 \mathrm{kPa}$ de pressão de sistema.

\begin{tabular}{|c|c|c|c|c|}
\hline Índice & $\mathbf{S}_{\mathbf{P} \mathbf{1}}$ & $\mathbf{S}_{\mathbf{P} \mathbf{1} \mathbf{x}} \mathbf{u}_{\mathbf{p}}$ & $\mathbf{1 0 0} \mathbf{x}\left(\mathbf{S}_{\mathbf{P} \mathbf{x}} \mathbf{\mathbf { u } _ { \mathbf { p } }} \mathbf{)}^{\mathbf{2}}\right.$ & $\begin{array}{c}\text { Contribuição do Transdutor } \\
\text { de Pressão da Entrada[\%] }\end{array}$ \\
\hline 1 & $-0,6$ & $-0,1$ & 1,1 & 12,5 \\
\hline 2 & $-0,6$ & $-0,1$ & 1,1 & 12,5 \\
\hline 3 & $-0,6$ & $-0,1$ & 1,1 & 12,5 \\
\hline 4 & $-0,6$ & $-0,1$ & 1,1 & 12,5 \\
\hline 5 & $-0,6$ & $-0,1$ & 1,1 & 12,4 \\
\hline 6 & $-0,6$ & $-0,1$ & 1,1 & 12,3 \\
\hline 7 & $-0,6$ & $-0,1$ & 1,3 & 13,4 \\
\hline 8 & $-0,6$ & $-0,1$ & 1,1 & 12,6 \\
\hline 9 & $-0,6$ & $-0,1$ & 1,1 & 12,5 \\
\hline 10 & $-0,7$ & $-0,1$ & 1,4 & 13,8 \\
\hline 11 & $-0,7$ & $-0,1$ & 1,3 & 13,5 \\
\hline
\end{tabular}


Tabela A86 - Coeficientes de sensibilidade para a pressão de saída a 4000 rpm, mistura 50/50 com 50 kPa de pressão de sistema.

\begin{tabular}{|c|c|c|c|c|}
\hline Índice & $\mathbf{S}_{\mathbf{P} \mathbf{2}}$ & $\mathbf{S}_{\mathbf{P} \mathbf{2} \times} \mathbf{u}_{\mathbf{p}}$ & $\mathbf{1 0 0} \mathbf{x}\left(\mathbf{S}_{\mathbf{P} \mathbf{2}} \mathbf{\mathbf { u } _ { \mathbf { p } }} \mathbf{)}^{\mathbf{2}}\right.$ & $\begin{array}{c}\text { Contribuição do Transdutor } \\
\text { de Pressão da Saída [\%] }\end{array}$ \\
\hline 1 & 1,6 & 0,3 & 7,8 & 87,5 \\
\hline 2 & 1,6 & 0,3 & 7,8 & 87,5 \\
\hline 3 & 1,6 & 0,3 & 7,8 & 87,5 \\
\hline 4 & 1,6 & 0,3 & 7,8 & 87,5 \\
\hline 5 & 1,6 & 0,3 & 7,8 & 87,6 \\
\hline 6 & 1,6 & 0,3 & 7,8 & 87,7 \\
\hline 7 & 1,6 & 0,3 & 8,2 & 86,6 \\
\hline 8 & 1,6 & 0,3 & 7,9 & 87,4 \\
\hline 9 & 1,6 & 0,3 & 7,8 & 87,5 \\
\hline 10 & 1,7 & 0,3 & 8,4 & 86,2 \\
\hline 11 & 1,7 & 0,3 & 8,3 & 86,5 \\
\hline
\end{tabular}

A Tabela A87 trás o cálculo da incerteza combinada para as medições feitas a 5000 rpm, com pressão de sistema de $50 \mathrm{kPa}$, utilizando a mistura de $50 \%$ etilenoglicol e 50\% água como fluido.

Tabela A87 - Incerteza combinada para 5000 rpm, mistura 50/50 com 50 kPa de pressão de sistema.

\begin{tabular}{|c|c|c|c|c|c|}
\hline Índice & $\mathbf{T}\left[{ }^{\circ} \mathbf{C}\right]$ & $\mathbf{P 1}[\mathbf{k P a}]$ & $\mathbf{P 2}[\mathbf{k P a}]$ & $\mathbf{D P}[\mathbf{k P a}]$ & $\begin{array}{c}\mathbf{u}_{\mathbf{c}} \\
{[\%]}\end{array}$ \\
\hline 1 & 85 & 41,3 & 141,3 & 100,0 & 2,6 \\
\hline 2 & 90 & 41,2 & 141,4 & 100,2 & 2,6 \\
\hline 3 & 93 & 41,1 & 141,6 & 100,5 & 2,6 \\
\hline 4 & 95 & 41,5 & 141,8 & 100,3 & 2,6 \\
\hline 5 & 97 & 41,4 & 141,8 & 100,4 & 2,6 \\
\hline 6 & 99 & 41,8 & 142,3 & 100,5 & 2,6 \\
\hline 7 & 101 & 41,6 & 141,7 & 100,1 & 2,6 \\
\hline 8 & 103 & 41,5 & 141,9 & 100,4 & 2,6 \\
\hline 9 & 105 & 41,2 & 139,0 & 97,8 & 2,6 \\
\hline 10 & 107 & 41,8 & 138,1 & 96,3 & 2,6 \\
\hline 11 & 110 & 41,7 & 133,5 & 91,8 & 2,7 \\
\hline
\end{tabular}

As Tabelas A88 e A89 apresentam o cálculo dos coeficientes de sensibilidade das medições das pressões de entrada e saída, respectivamente. A primeira coluna referencia a seqüência de medição. $A$ segunda, terceira e quarta coluna contém a informação para cada um dos 
componentes da Eq. 13. A quinta coluna traz a contribuição para a incerteza combinada.

Tabela A88 - Coeficientes de sensibilidade para a pressão de entrada a $5000 \mathrm{rpm}$, mistura 50/50 com $50 \mathrm{kPa}$ de pressão de sistema.

\begin{tabular}{|c|c|c|c|c|}
\hline Índice & $S_{P_{1}}$ & $S_{p 1 \times} u_{p}$ & $100 \times\left(S_{P_{1} \times} u_{p}\right)^{2}$ & $\begin{array}{l}\text { Contribuição do Transdutor } \\
\text { de Pressão da Entrada[\%] }\end{array}$ \\
\hline 1 & $-0,4$ & $-0,1$ & 0,5 & 7,9 \\
\hline 2 & $-0,4$ & $-0,1$ & 0,5 & 7,8 \\
\hline 3 & $-0,4$ & $-0,1$ & 0,5 & 7,8 \\
\hline 4 & $-0,4$ & $-0,1$ & 0,5 & 7,9 \\
\hline 5 & $-0,4$ & $-0,1$ & 0,5 & 7,9 \\
\hline 6 & $-0,4$ & $-0,1$ & 0,5 & 7,9 \\
\hline 7 & $-0,4$ & $-0,1$ & 0,5 & 7,9 \\
\hline 8 & $-0,4$ & $-0,1$ & 0,5 & 7,9 \\
\hline 9 & $-0,4$ & $-0,1$ & 0,5 & 8,1 \\
\hline 10 & $-0,4$ & $-0,1$ & 0,6 & 8,4 \\
\hline 11 & $-0,5$ & $-0,1$ & 0,6 & 8,9 \\
\hline
\end{tabular}

Tabela A89 - Coeficientes de sensibilidade para a pressão de entrada a $5000 \mathrm{rpm}$, mistura 50/50 com $50 \mathrm{kPa}$ de pressão de sistema.

\begin{tabular}{|c|c|c|c|c|}
\hline Índice & $\mathbf{S}_{\mathbf{P} \mathbf{2}}$ & $\mathbf{S}_{\mathbf{P} \mathbf{x}} \mathbf{u}_{\mathbf{p}}$ & $\mathbf{1 0 0} \mathbf{x}\left(\mathbf{S}_{\mathbf{P} \mathbf{x}} \mathbf{\mathbf { u } _ { \mathbf { p } }} \mathbf{)}\right.$ & $\begin{array}{c}\text { Contribuição do Transdutor } \\
\text { de Pressão da Saída [\%] }\end{array}$ \\
\hline 1 & 1,4 & 0,2 & 6,0 & 92,1 \\
\hline 2 & 1,4 & 0,2 & 6,0 & 92,2 \\
\hline 3 & 1,4 & 0,2 & 6,0 & 92,2 \\
\hline 4 & 1,4 & 0,2 & 6,1 & 92,1 \\
\hline 5 & 1,4 & 0,2 & 6,0 & 92,1 \\
\hline 6 & 1,4 & 0,2 & 6,1 & 92,1 \\
\hline 7 & 1,4 & 0,2 & 6,1 & 92,1 \\
\hline 8 & 1,4 & 0,2 & 6,0 & 92,1 \\
\hline 9 & 1,4 & 0,2 & 6,1 & 91,9 \\
\hline 10 & 1,4 & 0,2 & 6,2 & 91,6 \\
\hline 11 & 1,5 & 0,3 & 6,4 & 91,1 \\
\hline
\end{tabular}




\section{ANEXO B \\ Propriedades das Misturas Água / Etilenoglicol Testado (as informações abaixo foram cedidas pela empresa Tirreno - Indústria e Comercio de Produtos Químicos Ltda.).}

FLUIDO PARA ARREFECIMENTO TIRRENO ORGANIC COOL 100
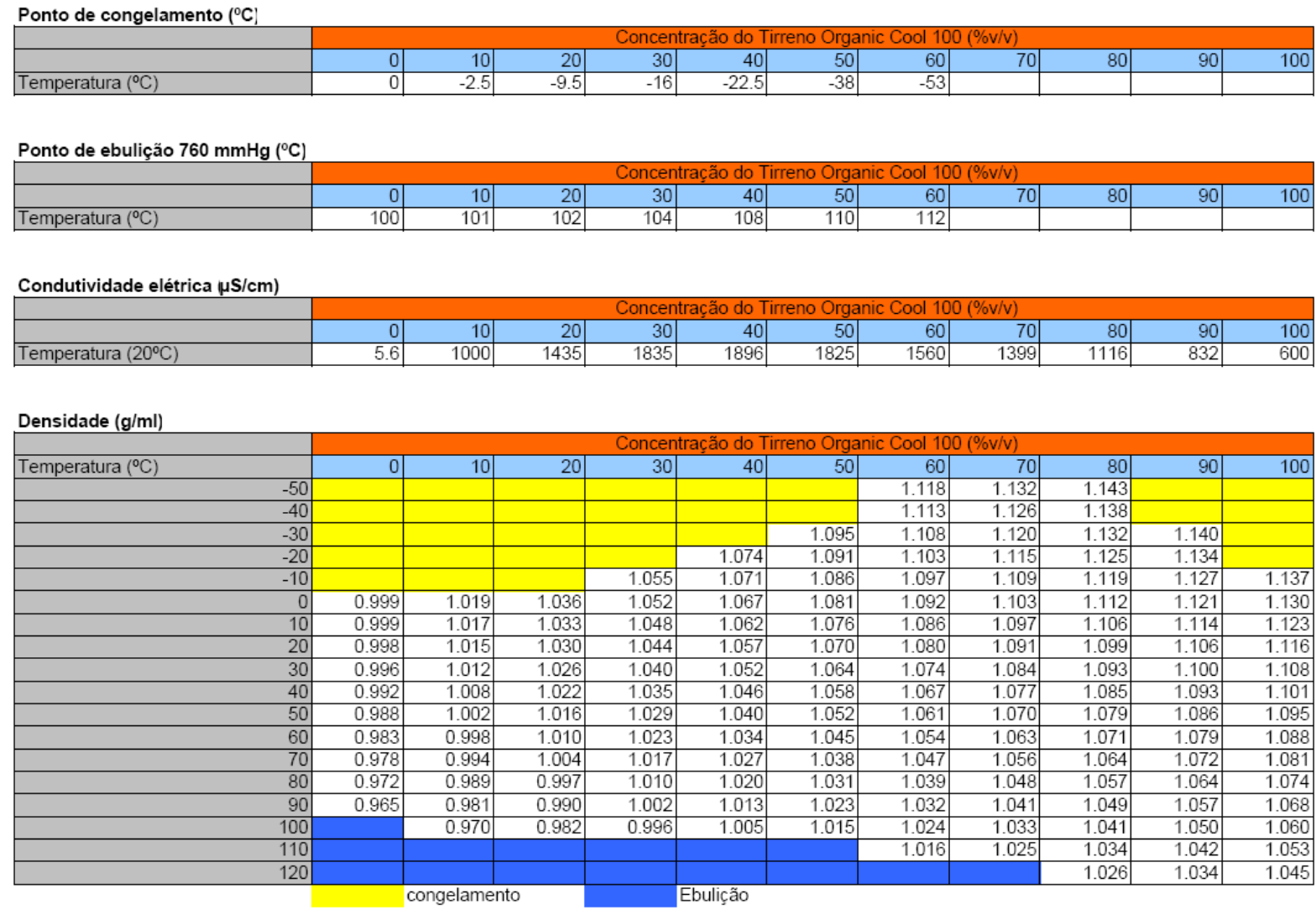

\begin{tabular}{|c|c|c|c|c|c|c|c|c|c|c|c|}
\hline & & & & Concen & ção do T & eno Orga & ic Cool 10 & $(\% v / v)$ & & & \\
\hline Temperatura $\left({ }^{\circ} \mathrm{C}\right)$ & 0 & 10 & 20 & 30 & 40 & 50 & 60 & 70 & 80 & 90 & 100 \\
\hline-50 & & & & & & & 172.0 & 285.6 & 254.7 & & \\
\hline-40 & & & & & & & 112.2 & 181.4 & 175.6 & & \\
\hline-30 & & & & & & 36.8 & 71.3 & 112.2 & 121.7 & 158.4 & \\
\hline-20 & & & & & 16.5 & 23.7 & 44.9 & 67.8 & 81.4 & 105.3 & \\
\hline-10 & & & & 6.7 & 11.0 & 15.3 & 29.8 & 39.6 & 53.6 & 68.6 & 89.4 \\
\hline 0 & & 2.6 & 3.6 & 4.9 & 7.4 & 9.9 & 18.4 & 24.5 & 34.5 & 44.4 & 57.2 \\
\hline 10 & 1.4 & 1.9 & 2.7 & 3.5 & 5.1 & 6.6 & 12.3 & 14.6 & 22.3 & 28.6 & 34.1 \\
\hline 20 & 1.1 & 1.5 & 2.0 & 2.6 & 3.4 & 4.5 & 8.2 & 9.8 & 14.8 & 19.7 & 23.6 \\
\hline 30 & 0.9 & 1.1 & 1.6 & 2.1 & 2.6 & 3.4 & 6.4 & 6.4 & 9.8 & 12.7 & 15.2 \\
\hline 40 & 0.8 & 1.0 & 1.3 & 1.7 & 1.9 & 2.5 & 3.6 & 4.8 & 6.2 & 9.3 & 11.1 \\
\hline 50 & 0.7 & 0.8 & 1.1 & 1.3 & 1.6 & 2.2 & 2.8 & 3.4 & 5.1 & 6.5 & 8.9 \\
\hline 60 & 0.6 & 0.7 & 1.0 & 1.2 & 1.5 & 1.9 & 1.9 & 2.9 & 4.0 & 4.7 & 6.5 \\
\hline 70 & 0.5 & 0.6 & 0.9 & 1.1 & 1.2 & 1.6 & 1.6 & 2.2 & 3.1 & 3.7 & 4.8 \\
\hline 80 & 0.4 & 0.6 & 0.8 & 0.9 & 1.2 & 1.4 & 1.3 & 1.7 & 2.4 & 2.9 & 3.9 \\
\hline 90 & 0.3 & 0.5 & 0.7 & 0.9 & 1.0 & 1.3 & 1.2 & 1.4 & 1.9 & 2.4 & 2.7 \\
\hline 100 & & 0.4 & 0.7 & 0.7 & 0.9 & 1.2 & 1.0 & 1.4 & 1.7 & 2.2 & 2.2 \\
\hline 110 & & & & & & & 0.8 & 1.2 & 1.5 & 1.9 & 1.9 \\
\hline 120 & & & & & & & & & 1.3 & 1.6 & 1.6 \\
\hline
\end{tabular}




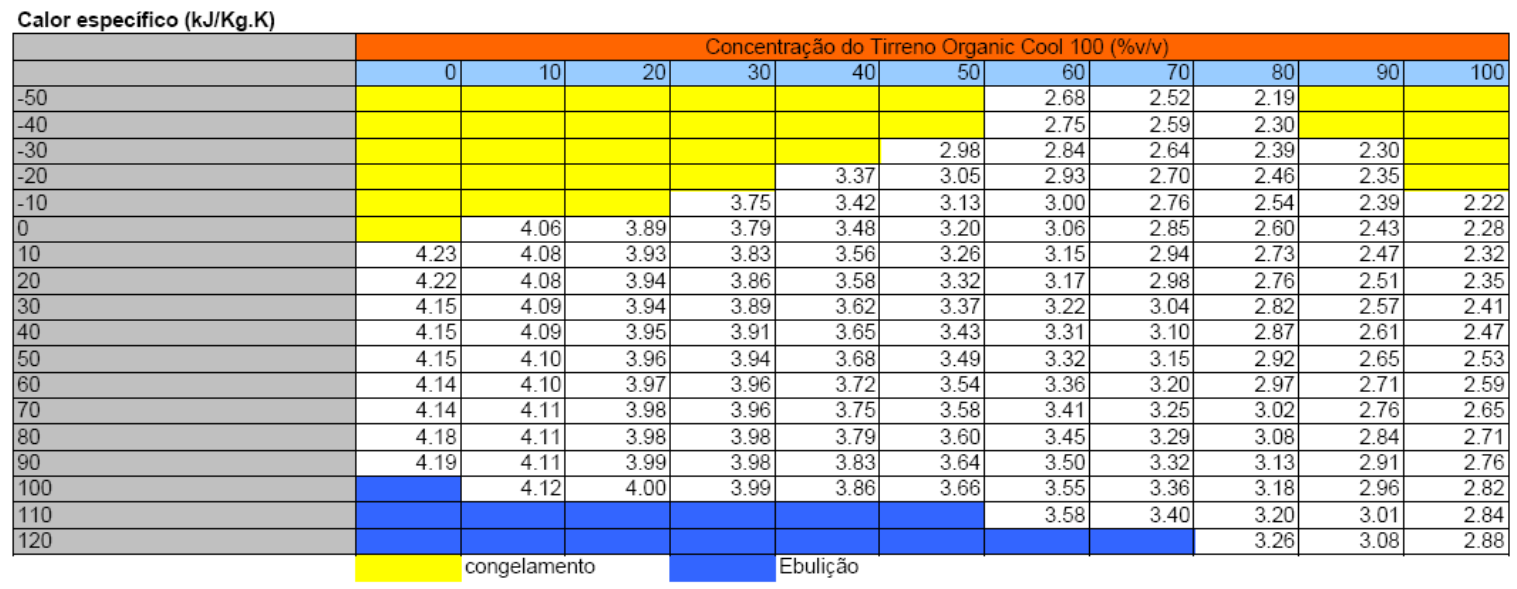

\begin{tabular}{|c|c|c|c|c|c|c|c|c|c|c|c|}
\hline \multirow[b]{2}{*}{ Temperatura $\left({ }^{\circ} \mathrm{C}\right)$} & \multicolumn{11}{|c|}{ Concentração do Tirreno Organic Cool $100(\% \mathrm{v} / \mathrm{v})$} \\
\hline & 0 & 10 & 20 & 30 & 40 & 50 & 60 & 70 & 80 & 90 & 100 \\
\hline 30 & 33.2 & 28.1 & 26.7 & 25.6 & 23.4 & 21.0 & 18.0 & 15.6 & 12.5 & 9.3 & 0.1 \\
\hline 40 & 56.7 & 52.2 & 48.8 & 44.6 & 41.1 & 37.5 & 32.9 & 26.5 & 20.3 & 15.3 & 0.1 \\
\hline 50 & 93.1 & 88.6 & 82.9 & 76.9 & 71.1 & 64.4 & 56.4 & 44.7 & 33.7 & 25.4 & 0.2 \\
\hline 60 & 149.2 & 143.2 & 134.7 & 126.1 & 117.4 & \begin{tabular}{l|l}
106.2 \\
\end{tabular} & 92.2 & 73.4 & 55.6 & 41.3 & 0.3 \\
\hline 70 & 233.1 & 224.0 & 211.8 & 198.7 & 186.0 & 168.5 & 145.5 & 116.3 & 87.1 & 64.2 & 0.5 \\
\hline 80 & 354.8 & 340.9 & 323.9 & 304.1 & 284.9 & 258.2 & 222.8 & 178.3 & 131.4 & 96.0 & 0.9 \\
\hline 90 & 525.9 & 505.8 & 482.0 & 453.7 & 424.6 & 384.1 & 331.6 & 265.1 & 194.8 & 139.6 & 1.6 \\
\hline 100 & 759.8 & 732.9 & 699.2 & 660.1 & 617.6 & 556.5 & 480.6 & 384.1 & 286.3 & 200.1 & 2.8 \\
\hline 110 & 1071.4 & 1037.9 & 990.5 & 936.9 & 877.0 & 788.3 & 680.4 & 544.4 & 413.5 & 286.1 & 5.0 \\
\hline 120 & 1477.5 & 1438.9 & 1372.4 & 1297.8 & 1215.2 & 1094.9 & 945.1 & 757.6 & 577.4 & 411.5 & 9.0 \\
\hline
\end{tabular}


PRESSÃO DE VAPOR

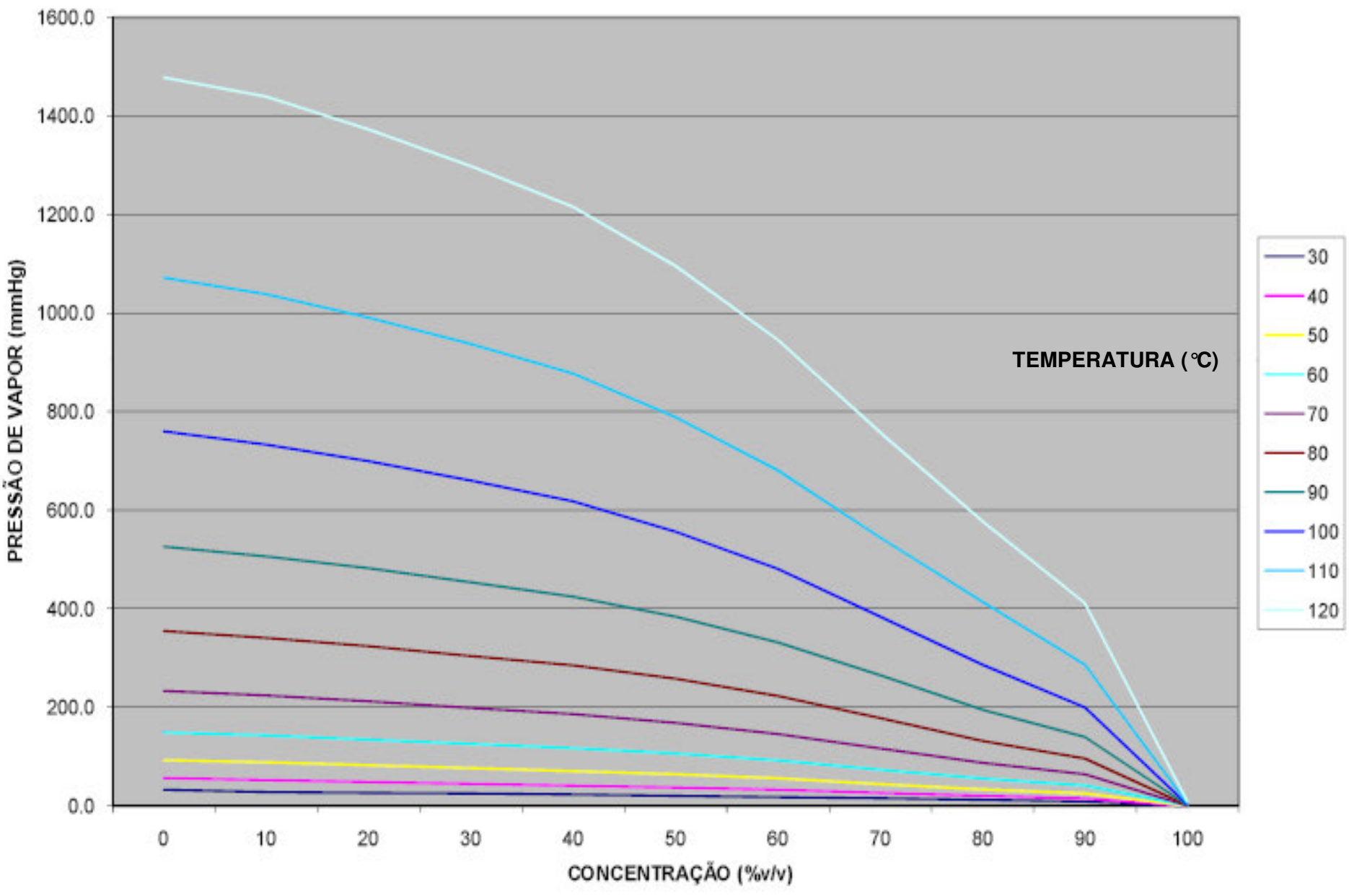




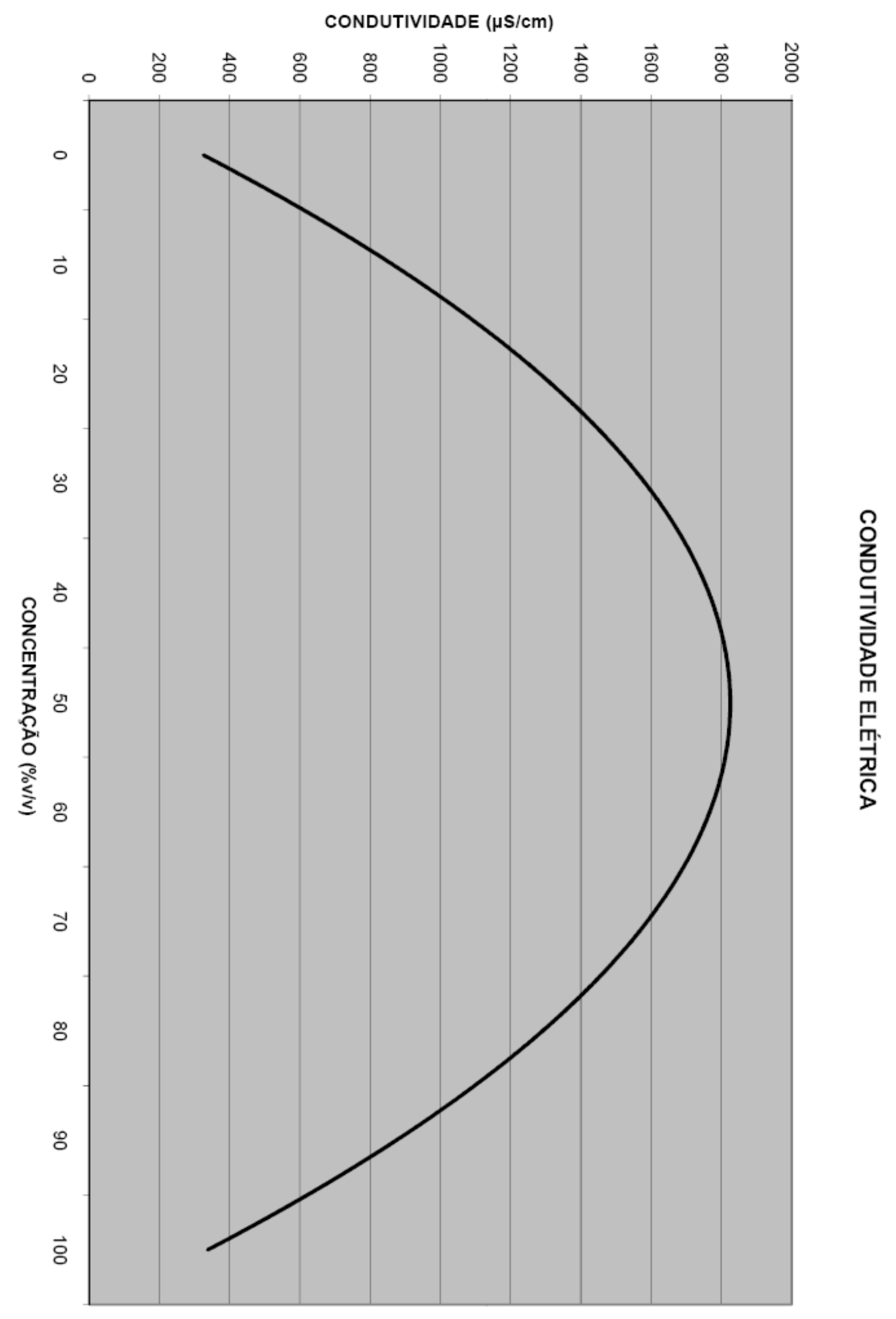


DENSIDADE

$\Xi$

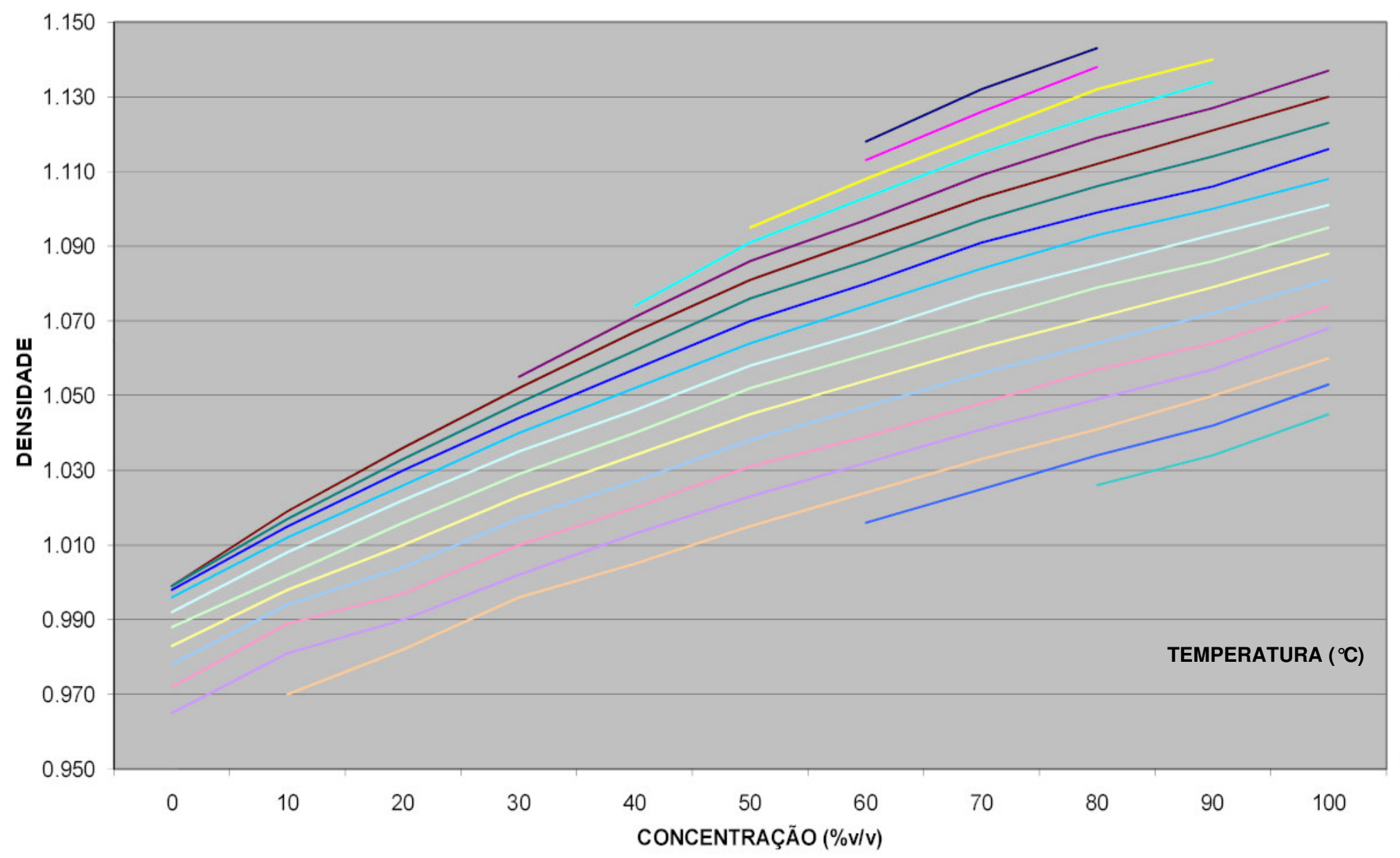

$\begin{array}{r}-50 \\ -40 \\ -30 \\ -20 \\ --10 \\ \hline-0 \\ -10 \\ \hline-20 \\ -30 \\ 40 \\ 50 \\ 60 \\ 770 \\ -80 \\ -90 \\ \hline 100 \\ \hline 110 \\ \hline 120 \\ \hline\end{array}$ 


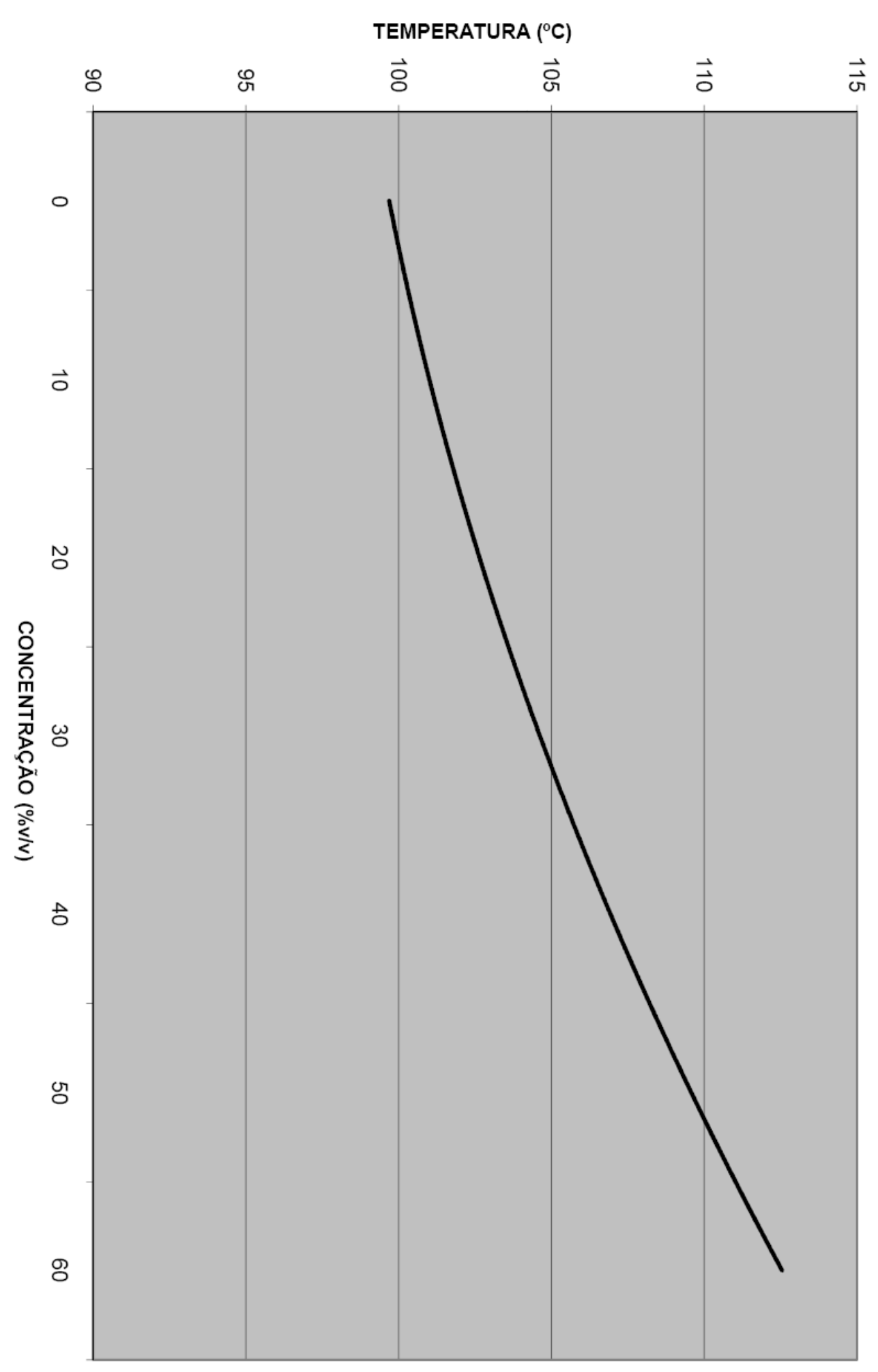

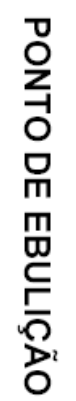


VISCOSIDADE CINEMÁTICA

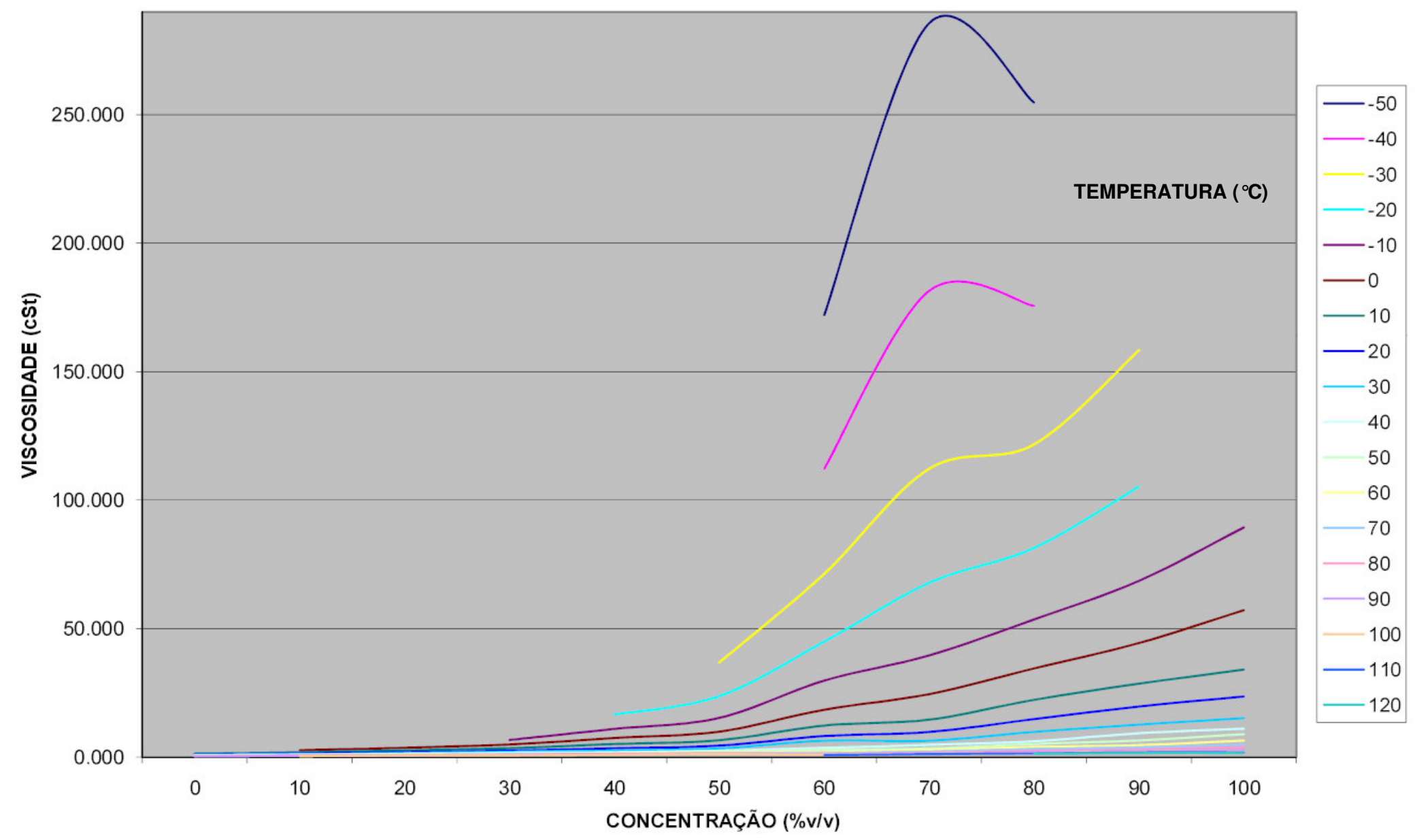




\section{REFERÊNCIAS BIBLIOGRÁFICAS}

BERCHICHE, N; FRANC, J.P.; MICHEL J.M. A Cavitation Erosion Model for Ductile Materials. France: Laboratoire des Ecoulements Géophysiques et Industriels, 2001.

CHAN, W. K. Correlation between cavitation type and cavitation erosion in centrifugal pumps. Int. J. Heat and Fluid Flow, Vol. 11, No. 3, September 1990.

HOFMANN, M.; STOFFEL, B. Experimental and Numerical Studies on a Centrifugal Pump with 2D Curved Blades in Cavitation Condition. Germany: Laboratory for Turbomachinery and Fluid Power, Darmstadt University of Technology, 2001.

LAUTERBORN, W.; LINDAU, O. Investigation of the Counterjet Developed in a Cavitation Bubble that Collapses Near a Rigid Boundary. Germany: Physikalisches Institut, Universit"at G"ottingen, 2001.

HATTORI, S.; NAKAO, E. Evaluation of Cavitation Erosion based on Erosion Particles. Japan: Department of Mechanical Engineering, Faculty of Engineering, Fukui University, 2001.

HUANG, K.D.; TZENG, S. C.; MA, W.P. Effects of anti-freeze concentration in the engine coolant on the cavitation temperature of a water pump. Applied Energy 79 (2004) 261-273. Elsevier, 2004.

DULAR, M.; STOFFEL, B.; SIROK, B. Development of a cavitation erosion model. Wear 261 (2006) 642-655. Elsevier, 2006.

CHAN, W. K., Detection of erosion in centrifugal pumps. Singapore: School of Mechanics and Production, Nanyang Technological Institute. Butterworth Publishers, 1988.

BRENNEM, C. E. Hydrodynamics of Pumps. USA: California Institute of Technology, Pasadena, California, Concepts ETI, Inc., 1994

TSUJIMOTO, Y. Simple Rules for Cavitation Instabilities in Turbomachinery. Japan: Engineering Science, Osaka University, 2001.

FARHAT, M.; AVELLAN, F. On the Detachment of a Leading Edge Cavitation. Switzerland: Laboratory For Hydraulic Machines. Swiss Federal Institute of Technology, 2001. 
ISHIDA, H.; NUNTADUSIT, C.; KIMOTO, H. Cavitation Bubble Behavior Near Solid Boundaries. Japan: Graduate School of Engineering Science, Osaka University, 2001

HATTORI, S.; GOTO, Y.; FUKUYAMAB, T. Influence of temperature on erosion by a cavitating liquid jet. Japan: Department of Mechanical Engineering, Faculty of Engineering, University of Fukui, 2005.

MOCHIZUKI, H.; YOKOTA, M.; HATTORI, S. Effects of materials and solution temperatures on cavitation erosion of pure titanium and titanium alloy in seawater. Wear 262 (2007) 522-528. Elsevier, 2006.

MARSCHALL, H. B.; MORCH, K. A.; KELLER, A. P. KJELDSEN, M. Cavitation Inception by Almost Spherical Solid Particles in Water. Germany: Department of Physics, Technical University of Denmark, 2001.

SOYAMA,Y.; KUMANO, H.; SAKA, M. A New Parameter to Predict Cavitation Erosion. Japan: Tohoku University, 2001.

FEDOTKIN, I.; YACHNO, O. Some Problems of Development of Cavitation Technologies for Industry Application. Ukraine: National Technical University, 2001.

KEGEL, T. M.; Basic Measurement Uncertainty. USA: Colorado Engineering Experiment Station. $71^{\text {st }}$ International School of Hydrocarbon Measurement, April,1996.

KIM, K.; LEE, J.; CHON, M.; YUN, J. A New Approach of Cavitation Criterion Analysis for Automotive Water Pumps. Korea: Seoul 2000 FISITA World Automotive Congress, 2000.

INMETRO. Vocabulário internacional de termos fundamentais e gerais de metrologia. 3. ed. Rio de Janeiro, 2003.

HOSNY, D. M. Real Time Cavitation Detection Method. SAE International. Paper 960878, 1996.

HOSNY, M. D.; HUDGENS, D.; COX, T. Cavitation Correlation to Fluid Media Properties. SAE International. Paper 960882, 1996.

BRENNEM, C. E. The Amazing World of Bubbles. USA: Engineering and Science, $n^{\circ} 1,2007$.

ELLIS, A.T.; BENJAMIN, T.B. The collapse of cavitation bubbles and the pressures thereby produced against solid boundaries. England: Phil. Trans. Roy. Soc., London, Ser. A, 260, 221-240, 1966. 
SHIMA, A.; TAKAYAMA, K.; TOMITA, Y.; OHSAWA, N. Mechanism of impact pressure generation from spark-generated bubble collapse near a wall. Japan: AIAA Journal, Vol. 21 (55-59), 1983.

IWAY, Y.; OKADA, T. General Mechanism of Cavitation Damage.

England: Cavitation of Hydraulic Machinery, World Scientific Publishing Co.

Pte. Ltd., 2000. 